\title{
Study of ordered hadron chains with the ATLAS detector
}

\author{
M. Aaboud et al. \\ (ATLAS Collaboration) \\ (Received 22 September 2017; published 29 November 2017)
}

\begin{abstract}
The analysis of the momentum difference between charged hadrons in high-energy proton-proton collisions is performed in order to study coherent particle production. The observed correlation pattern agrees with a model of a helical QCD string fragmenting into a chain of ground-state hadrons. A threshold momentum difference in the production of adjacent pairs of charged hadrons is observed, in agreement with model predictions. The presence of low-mass hadron chains also explains the emergence of charge-combination-dependent two-particle correlations commonly attributed to Bose-Einstein interference. The data sample consists of $190 \mu \mathrm{b}^{-1}$ of minimum-bias events collected with proton-proton collisions at a center-of-mass energy $\sqrt{s}=7 \mathrm{TeV}$ in the early low-luminosity data taking with the ATLAS detector at the LHC.
\end{abstract}

DOI: 10.1103/PhysRevD.96.092008

\section{INTRODUCTION}

Studies of correlated hadron production are an important source of information about the early stages of hadron formation, not yet understood from the theory of strong interactions. Although experimental high-energy physics employs several phenomenological models of hadronization that describe the formation of jets with remarkable accuracy, correlation phenomena are more elusive. In particular, the observed excess of nearby equally charged hadronscommonly attributed to Bose-Einstein interference-has never been satisfactorily reproduced by Monte Carlo (MC) models, despite several decades of intensive measurements. Furthermore, dedicated studies of these correlations in $W W$ production at LEP2 did not confirm the expected presence of correlations between hadrons originating from different color-singlet sources [1].

Recently, it was pointed out that correlations between likesign hadrons arise in the causality-respecting model of quantized fragmentation of a three-dimensional QCD string [2], as a consequence of coherent hadron emission [3]. The topology of the string and the causal constraint implemented in this model define the mass spectrum and the correlation pattern of emitted hadrons. This analysis investigates observables sensitive to predictions of the quantized string model. The experimental technique is focused on the extraction of a signal from correlated hadron pairs and triplets.

The paper is organized as follows. Section II contains a brief overview of phenomenological aspects of the quantized three-dimensional QCD string. Section III recounts the

*Full author list given at the end of the article.

Published by the American Physical Society under the terms of the Creative Commons Attribution 4.0 International license. Further distribution of this work must maintain attribution to the author(s) and the published article's title, journal citation, and DOI. observable features of the model and outlines the strategy of the analysis. Section IV describes the ATLAS detector. The data selection and MC event generators are described in Section V. Section VI shows the measured data. Correction of the data to the particle level is described in Sec. VII. Section VIII contains the results and the studies of systematic uncertainties. Section IX is devoted to the interpretation of results, and Sec. X contains concluding remarks.

\section{PHENOMENOLOGY OF QCD STRING FRAGMENTATION}

The Lund string fragmentation model [4], which is implemented in the PYTHIA event generator [5], uses a one-dimensional string to model the QCD confinement. The string is broken randomly by the production of a new quark-antiquark pair (or a pair of diquarks if baryons are to be produced). Hadron four-momenta are determined by the relative position and timing of adjacent breakup vertices. Hadrons sharing a common breakup vertex are called adjacent hadrons. The model imposes a spacelike distance between the vertices in order to produce hadrons with a positive (physical) mass. Despite the absence of a causal connection between vertices, the adjacent string breakups cannot be treated as random because they define the mass of the created hadron. The mass spectrum is enforced in the model by adding the mass constraint to the kinematics of the string decay, using hadron masses and widths as external parameters. The model relies on the concept of quantum tunneling to generate the intrinsic transverse momenta of hadrons; the partons created in the string breakup are assigned a transverse momentum with a constant azimuthal distribution and with a magnitude drawn randomly according to a Gaussian distribution with a tunable width. Local charge and momentum conservation hold in the breakup vertex, but according to the model, there are no correlations between nonadjacent hadrons in the string's transverse plane. 

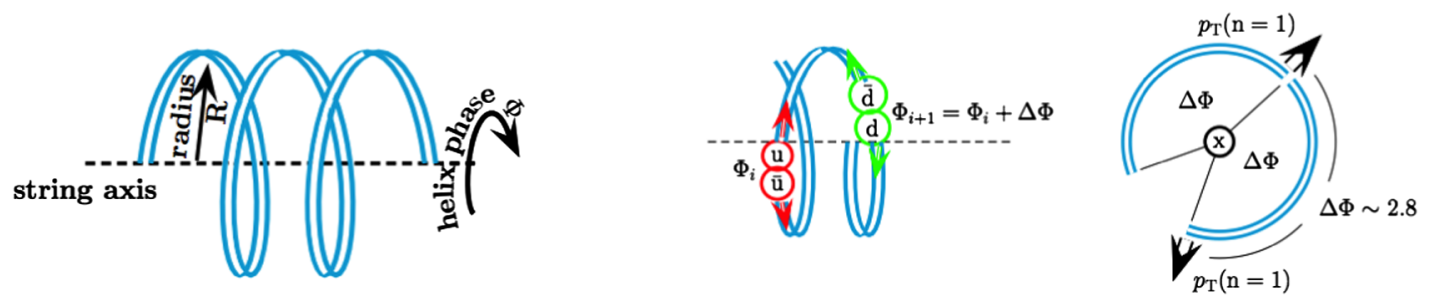

FIG. 1. Left: Parametrization of the helical shape of the QCD string. Middle: In quantized string fragmentation, the breakup points are separated by the quantized helix phase difference $\mathrm{n} \Delta \Phi, n=1$ for the ground-state pion. Right: The shape of the QCD string is reflected in the momentum distribution of emitted hadrons. The intrinsic transverse momentum of hadrons $p_{\mathrm{T}}(n)$ is quantized (see the Appendix). The azimuthal angle between intrinsic transverse momenta of adjacent ground-state pions is equal to $\Delta \Phi$.

The one-dimensional string serves as an approximation for a more complex QCD field shape, which may be similar to a thin vortex of a type-II superconductor. The possibility of understanding the shape of a QCD string in three dimensions was first studied in Ref. [3] with the goal of investigating effects stabilizing the end of the parton shower cascade. On the basis of angular properties of gluon emission under helicity conservation, the authors of Ref. [3] concluded that collinear gluon emissions are absent. On the basis of optimal packing of soft noncollinear gluon emissions, it was deduced that the shape of the QCD string should be helixlike.

The fragmentation in the transverse plane changes substantially when a one-dimensional string is replaced by a three-dimensional string and quantum tunneling is replaced by gluon splitting into a quark-antiquark pair with negligible momentum in the rest frame of the string stretched between the color-connected partons. Fragmentation of such a string generates intrinsic transverse momentum that depends on the folding of the string and implies azimuthal correlations between hadrons. Azimuthal correlations compatible with the helical shape of the QCD string have been observed by ATLAS [6].

A fragmentation model working with a threedimensional string enables cross-talk between breakup vertices to be introduced. When the causal constraint is imposed on the fragmentation of a helical QCD string described by radius $R$ and phase $\Phi$ (Fig. 1), the mass spectrum of light mesons is reproduced by a string breaking in regular $\Delta \Phi$ intervals. A fit of the mass spectrum of pseudoscalar mesons indicates a rather narrow radius of the helical string $(\kappa R=68 \pm 2 \mathrm{MeV}$, where $\kappa \sim 1 \mathrm{GeV} / \mathrm{fm}$ is the string tension) and a quantized phase difference $\Delta \Phi=$ $2.82 \pm 0.06[2]$.

The effective quantization of the string fragmentation predicts correlations between pairs of hadrons produced along the string, as a function of their rank difference $r .{ }^{1}$ Correlations can be studied with help of the momentum difference $Q$,

\footnotetext{
${ }^{1}$ The rank refers to the ordering of hadrons along the string; adjacent pairs have rank difference 1 .
}

$$
Q_{i j}=\sqrt{-\left(p_{i}-p_{j}\right)^{2}}
$$

where $p_{i}, p_{j}$ stand for the four-momenta of particles forming the pair. The numerical values of the predicted momentum difference separating pairs of ground-state pions ${ }^{2}$ with rank differences up to 5 are given in Table I. Predictions are calculated in the limit of a locally homogeneous string field with regular helix winding, which implies a vanishing longitudinal momentum difference between pions in the chain. Adjacent pions are produced with an intrinsic transverse momentum difference of $\sim 266 \mathrm{MeV}$, which can be seen as a quantum threshold for the production of adjacent hadrons. In a chain of adjacent charged pions, local charge conservation allows for the production of pairs of pions with equal charge for even rank differences $(r=2,4, \ldots)$ and opposite charge for odd rank differences $(r=1,3, \ldots)$ only. The low- $Q$ region $(Q<100 \mathrm{MeV})$ is populated by pairs with $r=2$.

Within the model, a chain of $n$ adjacent ground-state pions has the smallest possible mass for a chain of $n$ adjacent hadrons. It can be calculated using the relation

$$
m_{n}=\sqrt{n^{2} m_{\pi}^{2}+\sum_{i \neq j} Q_{i j}^{2}},
$$

where $m_{\pi}=m_{n=1}$ is the pion mass and $Q_{i j}$ stands for the momentum difference of pairs of hadrons forming the chain. Further information about the calculation of model predictions is provided in Appendix A.

\section{OBSERVABLE QUANTUM PROPERTIES OF STRING FRAGMENTATION}

The analysis uses the two-particle correlations measured for like-sign and opposite-sign hadron pairs to study the momentum difference between adjacent hadrons. The possible connection between the enhanced production of equally charged pions at low $Q$ and the production of

\footnotetext{
${ }^{2}$ The term ground-state pion denotes the lightest hadron state formed by a string piece with a helix phase difference $\Delta \Phi$, with a causal relation imposed on the end point breakup vertices.
} 
TABLE I. The expected momentum difference between hadrons formed by fragmentation of a homogeneous string into a chain of ground-state pions, in the quantized helix string model (see the Appendix). The 3\% uncertainty is derived from the precision of the fit of the mass spectrum of light pseudoscalar mesons $\left(\pi, \eta, \eta^{\prime}\right)[2]$.

\begin{tabular}{lccccc}
\hline \hline Pair rank difference $r$ & 1 & 2 & 3 & 4 & 5 \\
\hline$Q$ expected $(\mathrm{MeV})$ & $266 \pm 8$ & $91 \pm 3$ & $236 \pm 7$ & $171 \pm 5$ & $178 \pm 5$ \\
\hline \hline
\end{tabular}

chains of adjacent ground-state pions is investigated. For that purpose, correlations are measured for a selection of exclusive hadron triplets designed to isolate the source of correlations (see Sec. III A) and compared in detail to the inclusively measured two-particle correlations $[7,8]$. The correlation function is defined in a way that facilitates the measurement of adjacent hadron pairs (see Sec. III B).

\section{A. Analysis strategy: Chain selection}

The shortest chain of hadrons from which the properties of a helix can be inferred experimentally is a chain of three charged hadrons $(+-+,-+-)$, labeled $3 h$. For the chain selection, it is sufficient to consider only qualitative predictions of the model. It is experimentally impossible to reconstruct the history of string fragmentation from the momenta of final-state particles only, since the rank difference of any given pair of hadrons is unknown a priori. However, according to the model, a chain of ground-state pions will have the lowest possible mass as compared to a chain of arbitrary hadrons, and the smallest momentum difference within the chain of charged ground-state pions should be carried by the pair of like-sign pions. Therefore, the chain selection procedure is defined, event by event, in the following way:

(1) Each measured particle is paired with the like-sign particle that minimizes the pair momentum difference $Q$ calculated from the measured three-momenta. The pion mass is assigned to all particles. ${ }^{3}$

(2) Each pair is supplemented with an oppositely charged particle chosen to minimize the triplet mass. The resulting three-particle system, $(+-+)$ or $(-+-)$, is labeled as chain in the following, as the charge-conservation constraint is applied to define the relative ordering of particles. The chain selection is further refined in order to avoid doublecounting of particle pairs. The following criteriarooted in the underlying physics picture of string fragmentation, illustrated in Fig. 2-are applied in an iterative way, preserving the configurations with lowest mass found so far.

\footnotetext{
${ }^{3}$ Throughout this paper, the pion mass is assigned to all charged particles in the data and in the MC simulation to reflect the absence of particle identification in the data.
}

(3) The association of particles is verified, in the order of increasing pair momentum difference. If a particle is associated with more than two different like-sign partners, the two pairs with smallest momentum difference are retained, and the remaining associations are discarded. A new search for a closest likesign partner is performed using the still-available particles. Since the algorithm allows a pair of likesign particles to be associated with two different chains, each protochain is assigned a weight of $w_{i}=$ 0.5 or $w_{i}=1$ accordingly, to prevent the doublecounting of identical chains.

(4) After completion of chains with opposite-sign hadrons, the association rate of all opposite-sign pairs in the chain selection is verified, in the order of increasing chain mass. According to the string fragmentation picture, a pair of adjacent hadrons can be shared by at most two adjacent triplet chains (Fig. 2). In the case where a pair of opposite-sign hadrons belongs to three or more selected chains, the two chains with lowest mass are retained, and a new search for an opposite-sign partner is performed for the other chains. If that search fails, the weight of the corresponding chain is set to zero. Zero weight is also assigned to incomplete chains if there are not enough particles in the event to construct triplets.

At the end of the procedure, the chain selection contains $n_{\mathrm{ch}}$ chains in an event with $n_{\mathrm{ch}}$ charged particles, and some of these chains are effectively eliminated, having zero weight. The requirement for the like-sign pair to carry the smallest momentum difference within the chain is not imposed in any way; only $\sim 1 / 3$ of selected chains contains such a configuration. Although the chain selection builds on generic properties of chains of ground-state hadrons by minimizing both the momentum difference for like-sign pairs and the mass of triplets, the numerical predictions of the helical string model are not used in the chain selection. For the sake of simplicity, the analysis is restricted to the study of triplet chains only.

\section{B. Analysis strategy: Definition of the correlation function}

In the picture of the string fragmentation, the number of pairs of adjacent hadrons or pairs of hadrons with a fixed rank difference is proportional to the number of charged particles in the sample, while the total number of particle pairs grows quadratically with particle multiplicity. The choice of the correlation function is therefore driven by the need to separate the signal from adjacent hadron pairs and the large combinatorial background.

In the fragmentation of a QCD string, the creation of adjacent like-sign pairs is forbidden by local charge conservation. For higher rank differences, the like-sign and unlike-sign pairs should be produced in equal amounts due to the random production of neutral hadrons in the 


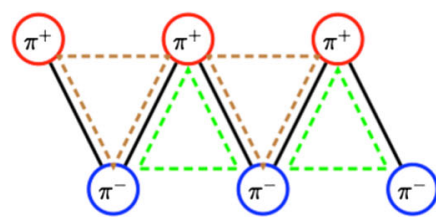

(a)

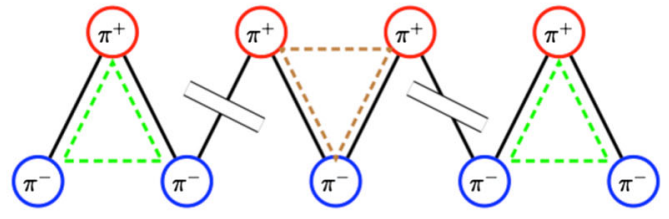

(b)

FIG. 2. A schematic view of a string fragmentation into (a) a long uninterrupted chain and (b) disconnected three-hadron chains. The circles represent charged hadrons, and the black lines indicate the ordering according to the string fragmentation history (they connect adjacent hadron pairs). Dashed triangles indicate the triplet chains. In the long uninterrupted chain (a), neighboring triplets share a common opposite-sign pair.

chain. It follows that the subtraction of like-sign pairs from the opposite-sign pairs is a suitable technique for isolating the signal from adjacent hadron pairs. The inclusive correlations are therefore assessed by the correlation function

$$
\Delta(Q)=\frac{1}{N_{\mathrm{ch}}}\left[N(Q)^{\mathrm{OS}}-N(Q)^{\mathrm{LS}}\right]
$$

where $N_{\text {ch }}$ stands for the number of charged particles in the sample and $N(Q)^{\mathrm{OS}}\left(N(Q)^{\mathrm{LS}}\right)$ denotes the inclusive spectrum of opposite-sign (like-sign) pairs in the sample:

$$
\begin{aligned}
N(Q)^{\mathrm{OS}} & =\sum_{k=1}^{N_{\mathrm{ev}}} \sum_{i, j=1, i \neq j}^{n_{\mathrm{ch}}^{k}} \delta\left(q_{i}+q_{j}\right) \delta\left(Q-Q_{i j}\right), \\
N(Q)^{\mathrm{LS}} & =\sum_{k=1}^{N_{\mathrm{ev}}} \sum_{i, j=1, i \neq j}^{n_{\mathrm{ch}}^{k}} \delta\left(q_{i}-q_{j}\right) \delta\left(Q-Q_{i j}\right) .
\end{aligned}
$$

The $\delta(x)=1-\operatorname{sign}^{2}(x)$ corresponds to the Kronecker delta with a continuous argument, which is 1 for $x=0$ and zero otherwise. $N_{\mathrm{ev}}$ stands for the number of events, $n_{\mathrm{ch}}^{k}$ is the number of charged particles in event $k$, and $q_{i}$ is the charge of particle $i$. The integral of the $\Delta(Q)$ distribution depends on the distribution of the total event charge only, with an upper limit of 0.5 for a sample of events with an equal number of negatively and positively charged particles. Experimentally, the restricted reconstruction acceptance creates a charge imbalance, which implies a larger loss of opposite-sign pairs compared to that of likesign pairs and hence diminishes the integral of $\Delta(Q)$ distribution.

The properties of the correlation function were verified on MC samples where no correlations were introduced beyond local charge and momentum conservation in the string breakup. The subtraction of inclusive pair distributions provides the same result as the extraction of true adjacent pairs, up to the uncertainty in the particle ordering generated by the presence of resonances decaying into three and more charged hadrons [see Fig. 8(a) for illustration]. This implies that the definition of the correlation function to a large extent compensates for not knowing the exact hadron ordering in the string fragmentation. Traditionally, correlation studies employ a ratio of $Q$ distributions rather than a difference, assuming incoherent or collective effects. Such an approach, however, does not eliminate the combinatorial background from the measurement, and therefore it is far less suitable for the measurement of the hadronization effects. A comparison of the two approaches is discussed in Sec. IX B.

In direct correspondence to Eq. (3), the correlations carried by exclusive three-hadron chains can be expressed as a sum over contributions from all chains with nonzero weight,

$$
\begin{aligned}
\Delta_{3 h}(Q)= & \frac{1}{N_{\mathrm{ch}}} \sum_{k=1}^{N_{\mathrm{ev}}} \sum_{i=1}^{n_{\mathrm{ch}}^{k}} w_{i}\left\{\frac{1}{2} \delta\left(Q-Q_{01}^{i}\right)+\frac{1}{2} \delta\left(Q-Q_{12}^{i}\right)\right. \\
& \left.-\delta\left(Q-Q_{02}^{i}\right)\right\}
\end{aligned}
$$

where each chain contributes with three entries: two for opposite-sign pairs at $Q_{01}, Q_{12}$ and one for the like-sign pair at $Q_{02}$ (the indices reflect charge ordering of particles in the chain). The $w_{i}$ stand for the weight factor of the $i$ th chain in the event.

The scaling of the opposite-sign pair contribution by $1 / 2$ in Eq. (5) is required for proper subtraction of random combinations; physicswise, it corresponds to a hypothesis of an uninterrupted chain of charged hadrons where neighboring triplets share an opposite-sign pair, Fig. 2(a). The estimate for disconnected triplets [Fig. 2(b)], where the opposite-sign pairs are not shared and should be counted with weight 1 , can be obtained from the measurement of $\Delta_{3 h}$ (after subtraction of random combinations), by rescaling the opposite-sign pair contribution-the positive part of the $\Delta_{3 h}(Q)$ spectrum - by a factor of 2 .

\section{Analysis strategy: Three-body decay}

Quantized fragmentation of the helical string into a chain of charged pions is expected to produce a distinct threebody decay pattern. The chain members are separated by a momentum difference that depends on their rank difference 
(Table I). For a triplet chain, such a signal can be studied with the help of a Dalitz plot. In analogy with studies of $\eta$ decay $[9,10]$, the Dalitz plot coordinates $(X, Y)$ are defined as

$$
X=\sqrt{3} \frac{T_{0}-T_{2}}{\Sigma_{i=0}^{2} T_{i}}, \quad Y=\frac{3 T_{1}}{\Sigma_{i=0}^{2} T_{i}}-1,
$$

where $T_{i}$ denotes the kinetic energy $E_{i}\left(m_{\pi}\right)-m_{\pi}$ of charge-ordered particles in the rest frame of the chain (particles 0 and 2 form the like-sign pair). The method of calculation of predictions of the helix-string model can be found in the Appendix [Eq. (A3)].

\section{ATLAS DETECTOR}

The ATLAS detector [11] covers almost the entire solid angle around the collision point with layers of tracking detectors, calorimeters, and muon chambers. For the measurements presented in this paper, the trigger system and the tracking devices are of particular importance. The following description corresponds to the detector configuration in the first LHC data-taking period (Run 1).

The ATLAS inner detector has full coverage in $\phi$ and covers the pseudorapidity range $|\eta|<2.5$. $^{4}$ It consists of a silicon pixel detector, a silicon strip detector (SCT), and a transition radiation tracker (TRT). These detectors are immersed in a $2 \mathrm{~T}$ axial magnetic field. The pixel, SCT, and TRT detectors have typical $r-\phi$ position resolutions of 10,17 , and $130 \mu \mathrm{m}$, respectively, and the pixel and SCT detectors have $r-z$ position resolutions of 115 and $580 \mu \mathrm{m}$, respectively. A track traversing the full radial extent would typically have 3 silicon pixel hits, 8 or more silicon strip hits, and more than 30 TRT hits.

The ATLAS detector has a three-level trigger system: level 1 (L1), level 2 (L2), and the event filter (EF). For this measurement, the L1 trigger relies on the beam pickup timing devices (BPTX) and the minimum-bias trigger scintillators (MBTS). The BPTX are composed of electrostatic beam pickups attached to the beam pipe at a distance $z= \pm 175 \mathrm{~m}$ from the center of the ATLAS detector. The MBTS are mounted at each end of the inner detector in front of the end cap calorimeter at $z= \pm 3.56 \mathrm{~m}$ and are segmented into eight sectors in azimuth and two rings in pseudorapidity $(2.09<|\eta|<2.82$ and $2.82<|\eta|<3.84)$. Data were taken for this analysis using the single-arm MBTS trigger, formed from BPTX and MBTS L1 trigger signals. The MBTS trigger was configured to require one hit above

\footnotetext{
${ }^{4}$ ATLAS uses a right-handed coordinate system with its origin at the nominal interaction point (IP) in the center of the detector and the $z$ axis along the beam pipe. The $x$ axis points from the IP to the center of the LHC ring, and the $y$ axis points upward. Cylindrical coordinates $(r, \phi)$ are used in the transverse plane, $\phi$ being the azimuthal angle around the beam pipe. The pseudorapidity is defined in terms of the polar angle $\theta$ as $\eta=-\ln \tan (\theta / 2)$.
}

threshold from either side of the detector. The MBTS trigger efficiency was studied with a separate prescaled L1 BPTX trigger, filtered to obtain inelastic interactions by inner detector requirements at L2 and the EF [12].

\section{DATA SELECTION AND MC EVENT GENERATORS}

Event and track selection are identical to those used in Refs. [7,12]. The data sample consists of $190 \mu \mathrm{b}^{-1}$ of minimum-bias events collected with proton-proton collisions at a center-of-mass energy $\sqrt{s}=7 \mathrm{TeV}$ in the early 2010 ATLAS data taking with negligible contribution from additional $p p$ collisions in the same bunch crossing. Events must:

(i) pass a single arm MBTS trigger,

(ii) have a primary vertex reconstructed with at least two associated tracks each with transverse momentum $\left(p_{\mathrm{T}}\right)$ above $100 \mathrm{MeV}$,

(iii) not have a second primary vertex reconstructed with more than three tracks,

(iv) have at least two good tracks, as defined below.

A reconstructed track passes the selection if it has:

(i) $p_{\mathrm{T}}>100 \mathrm{MeV}$ and lies in the pseudorapidity range $|\eta|<2.5$;

(ii) absolute values of transverse and longitudinal impact parameters below $1.5 \mathrm{~mm}$, with respect to the event primary vertex;

(iii) a hit in the first pixel layer when expected and at least one pixel hit in total;

(iv) at least two (for $p_{\mathrm{T}}>100 \mathrm{MeV}$ ), four (for $p_{\mathrm{T}}>$ $200 \mathrm{MeV}$ ), or six (for $p_{\mathrm{T}}>300 \mathrm{MeV}$ ) SCT hits;

(v) a fit probability above 0.01 for $p_{\mathrm{T}}>10 \mathrm{GeV}$.

The sample contains $\sim 10$ million events and over 200 million reconstructed tracks. The detector effects are evaluated using a PYTHIA6.421 [13] event sample with parameter values from the MC09 tune [14], fully simulated [15] and reconstructed using the standard ATLAS reconstruction chain [16]. According to MC estimates, the selected set of reconstructed charged particles consists of $86 \%$ pions, $9.5 \%$ kaons, $4 \%$ baryons, and $0.5 \%$ leptons, while the fraction of nonprimary particles is $2.3 \%$. Primary particles are defined as all particles with a lifetime longer than $0.3 \times 10^{-10} \mathrm{~s}$ originating from the primary interaction or from subsequent decay of particles with a shorter lifetime.

Correlation effects that are studied in the present analysis are absent in hadronization models, and therefore the analysis does not rely on MC predictions. For illustration, the data are compared with a representative set of hadronization models including PYTHIA8 (4C tune [17]), HERWIG++ $[18,19]$ and EPOs [20].

\section{CORRELATION FUNCTIONS AT DETECTOR LEVEL}

The inclusive distribution $\Delta(Q)$-as obtained from reconstructed data-is shown in Fig. 3. It shows an 


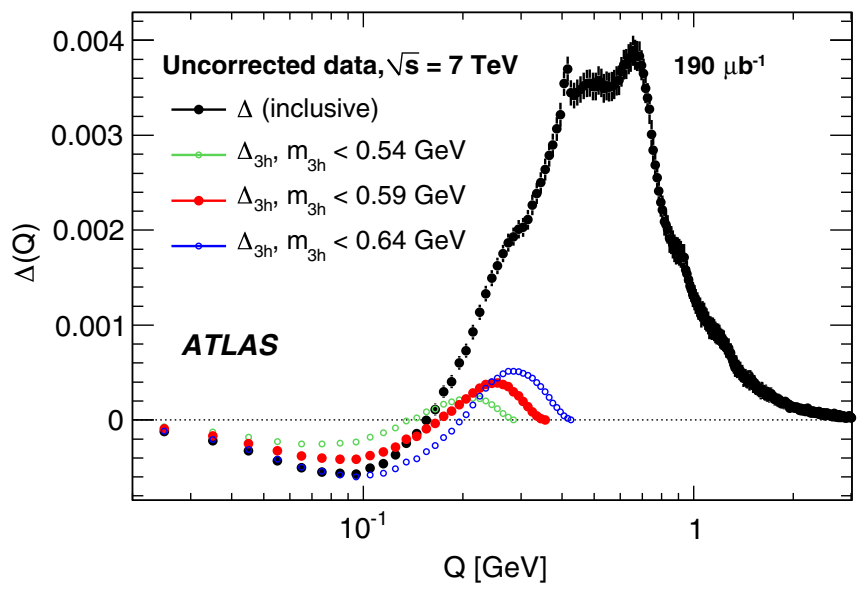

FIG. 3. Comparison of $\Delta_{3 h}$ (constructed from pairs belonging to the exclusive three-particle chain selection) with $\Delta$ (inclusive two-particle distribution), as a function of the upper limit on the mass of selected three-hadron chains for uncorrected data.

enhanced production of like-sign hadron pairs at low $Q$, visible as a negative value. The effect is quantified by the correlation strength (CS) defined as the absolute value of the integral of the negative part of the $\Delta(Q)$ distribution

$$
\mathrm{CS}=-\int_{\Delta<0} \Delta(Q) \mathrm{d} Q .
$$

The correlation function measured for three-hadron chains, $\Delta_{3 h}(Q)$, exhibits a shape similar to $\Delta(Q)$ at low $Q$ (Fig. 3). However, the shape of the $\Delta_{3 h}(Q)$ also depends on the upper mass limit, $m_{3 h}^{\text {cut }}$, imposed on the chain selection. The chain correlation strength (CCS) is defined as

$$
\operatorname{CCS}\left(m_{3 h}^{\text {cut }}\right)=-\int_{\Delta_{3 h}<0} \Delta_{3 h}(Q) \mathrm{d} Q, \text { for } m_{3 h}<m_{3 h}^{\text {cut }} .
$$

A comparison of the two distributions suggests that the selection of triplet hadron chains with minimized mass may contain the source of two-particle correlations observed in the inclusive sample, but this information is indicative only at this stage due to the absence of unfolding of detector effects that affect the triplet selection more than the inclusive two-particle spectra. The average reconstruction efficiency for a pair of charged particles is $\sim 50 \%$, and for a triplet of charged particles, it drops to $\sim 35 \%$.

\section{CORRECTION TO PARTICLE LEVEL}

The data are corrected for detector effects within the acceptance requirements $\left(p_{\mathrm{T}}>100 \mathrm{MeV},|\eta|<2.5\right)$ following the correction procedure established in Ref. [12]. The track reconstruction inefficiency, the presence of nonprimary tracks, and the migration of tracks across the acceptance boundaries are corrected for by applying track-based weights. The vertex and trigger efficiency is corrected for with an event-based weight. The dominant component of the uncertainty of the track weighting factors comes from the dependence on the generated MC sample. Anticipating a strong contribution to the measurement from the low- $p_{\mathrm{T}}$ region, the fully simulated PYTHIA6 sample is split into nondiffractive and diffractive components. The former is used to calculate track-based weights, and the latter is used to evaluate the uncertainty of the correction in the low- $p_{\mathrm{T}}$ region. The observed difference for the inclusive $Q$ spectra [Eq. (4)] is $\sim 10 \%$, without a significant $Q$ dependence. The uncertainty of the track weighting factors due to the imperfect detector description is evaluated using MC samples simulated and reconstructed with a $10 \%$ increase of material in the inner detector, which corresponds to the uncertainty of the detector description (Ref. [12]). The observed change of the inclusive $Q$ does not exceed $2 \%$. Both effects are combined and translated into an effective single-track weight uncertainty of $5 \%$.

The study of hadron pairs is sensitive to detector effects related to the proximity of reconstructed tracks that are not explicitly included in the track-based weights; the reduced reconstruction efficiency for pairs of tracks with a very low opening angle and the correlated nonprimary particle production are taken into account via additional correction factors and additional systematic uncertainty. Both are parametrized in MC samples as a function of the opening angle between particles, and the parametrization is convolved with the opening-angle distribution obtained from the data. The subtraction of the like-sign hadron pair spectrum from the opposite-sign pair spectrum implies a large cancellation of these effects and cancellation of systematic effects in general. This renders the experimental technique very robust. The uncertainty of track-based weights is effectively removed from the integral of the $\Delta(Q)$ distribution thanks to the appropriate choice of the normalization. The residual bias of the pair-correction procedure is evaluated in $\mathrm{MC}$ samples and added to the pair reconstruction systematic uncertainty. Figure 4(a) shows the $Q$ dependence of the $\Delta(Q)$ uncertainty related to the combined pair reconstruction uncertainty and its components. The uncertainty is larger than (or comparable to) the correction observed in the MC simulation. The corrected inclusive two-particle $Q$ spectra for like-sign and oppositesign pairs used in this analysis, $N(Q)^{\mathrm{LS}}$ and $N(Q)^{\mathrm{OS}}$, were published earlier [7] and can be obtained from Ref. [8].

The correction of triplet chains needs to take into account the impact of the track reconstruction inefficiency on the chain selection algorithm described in Sec. III A. The correction for this effect is handled by the HBOM correction technique [21]. In the HBOM method, the reconstructed tracks are randomly removed from the sample according to the detector reconstruction efficiency, which is parametrized in terms of charged-particle $p_{\mathrm{T}}$ and pseudorapidity. Hence, the HBOM iteration corresponds to the 


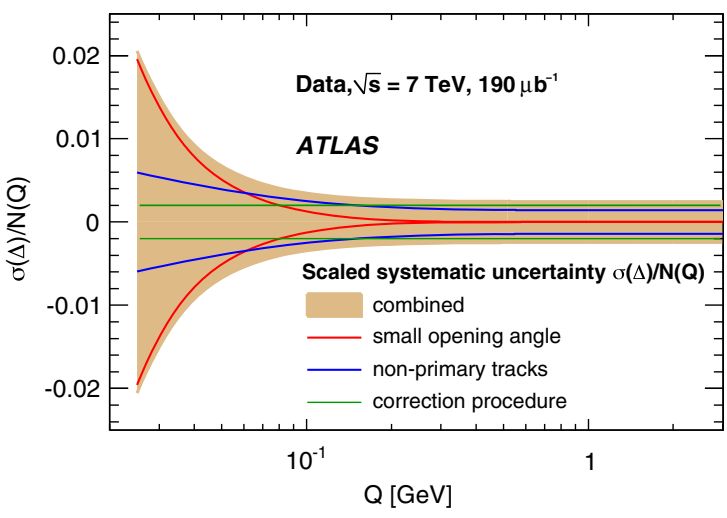

(a)

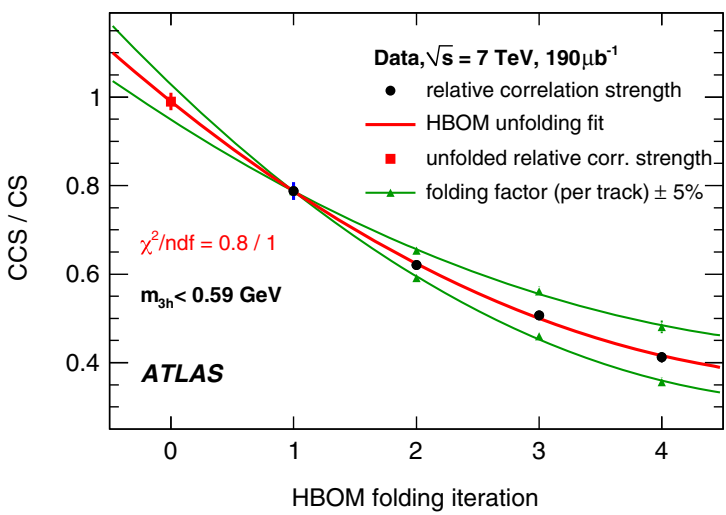

(b)

FIG. 4. (a) Components of the systematic uncertainty of $\Delta(Q)$ related to the reconstruction of pairs of tracks normalized to the combined inclusive spectrum $N(Q)=N(Q)^{\mathrm{OS}}+N(Q)^{\mathrm{LS}}$. (b) HBOM fit (red curve), which provides the corrected relative correlation strength (red square marker). The systematic uncertainty is split into pair reconstruction uncertainty (error bar superimposed over the raw data point) and the variation of track folding factors (green lines).

folding of the data with detector effects. The chain selection is repeated using remaining tracks, for several HBOM folding iterations, and the results are used to establish the functional dependency of the measured quantity on detector effects. The parametrization of this dependency is then extrapolated to the detector-effect free (zeroth folding iteration) result. The statistical correlations between HBOM folding iterations are suppressed by resampling the probability to retain a given track in each folding iteration independently.

The systematic uncertainty of the HBOM method is extracted directly from the data by performing an additional HBOM unfolding to the detector level, where the detectorlevel data are taken as the reference and compared to the HBOM unfolding based on remaining folding iterations.

The relative correlation strength CCS/CS is unfolded using the HBOM technique as shown in Fig. 4(b) for a chain mass-limit value of $0.59 \mathrm{GeV}$. The raw data and three HBOM folding iterations are fitted using a second-order polynomial. Within the CS and CCS integral ranges, statistical uncertainties of CS and CCS are highly correlated, and therefore the statistical uncertainties of input points are calculated assuming maximal overlap between samples. The extrapolation of the fitted function to the zeroth folding iteration yields the unfolded result and the statistical error estimate. The systematic uncertainty is split into two components indicated on the plot: the combined pair reconstruction uncertainty that is considered fully correlated between HBOM iterations and the uncertainty of the folding factors, equal to the uncertainty of trackbased weights discussed above.

The analysis employs two different techniques in order to correct the shape of $\Delta_{3 h}$. The generic correction consists in the evaluation of the fraction and of the shape of chains affected by track recombination. A chain which does not lose any track in a given HBOM folding iteration is labeled as a "surviving" chain. The chain survival probability for the $i$ th HBOM iteration $(i>1)$ is calculated as the number of chains surviving from the $(i-1)$ th iteration divided by the number of chains selected in the $i$ th iteration. Figure 5(a) shows, for a fixed chain mass limit, the chain survival probability as measured for three consecutive HBOM folding iterations. The chain recombination probability is complementary to the chain survival probability. It serves as an input for the unfolding of the recombination probability at the detector level ( $\sim 34 \%$ for a chain mass limit of $0.59 \mathrm{GeV})$. The distribution of $\Delta_{3 h}(Q)$ for the recombined chains is obtained as a difference between the $\Delta_{3 h}(Q)$ distribution obtained in a given folding iteration and the $\Delta_{3 h}(Q)$ distribution of chains surviving from the previous iteration. The shape of $\Delta_{3 h}(Q)$ for the recombined chains, together with the normalization obtained from the unfolded recombination rate, is used to produce an estimate of the contribution of recombined chains to the detector-level measurement. After subtraction of the recombined chains, the raw data are unfolded using track-based weight factors, in analogy with the unfolding of inclusive pair spectra.

The second technique is designed to unfold the parametrized shape of $\Delta_{3 h}(Q)$, which is well approximated by a sum of two Gaussian functions describing the residual difference between the opposite-sign and like-sign pair content,

$$
\begin{aligned}
\Delta_{3 \mathrm{~h}}(Q)= & f_{\mathrm{LS}}\left(Q ; Q_{\mathrm{LS}}, \sigma_{\mathrm{LS}}\right)+f_{\mathrm{OS}}\left(Q ; Q_{\mathrm{OS}}, \sigma_{\mathrm{OS}}\right) \\
= & n_{\mathrm{LS}} \exp \left(\frac{-\left(Q-Q_{\mathrm{LS}}\right)^{2}}{2 \sigma_{\mathrm{LS}}^{2}}\right) \\
& +n_{\mathrm{OS}} \exp \left(\frac{-\left(Q-Q_{\mathrm{OS}}\right)^{2}}{2 \sigma_{\mathrm{OS}}^{2}}\right),
\end{aligned}
$$

where $n_{\mathrm{LS}}<0 \quad\left(n_{\mathrm{OS}}>0\right)$ are scale factors, $Q_{\mathrm{LS}}\left(Q_{\mathrm{OS}}\right)$ indicate the position of Gaussian peaks, and $\sigma_{\mathrm{LS}}\left(\sigma_{\mathrm{OS}}\right)$ 


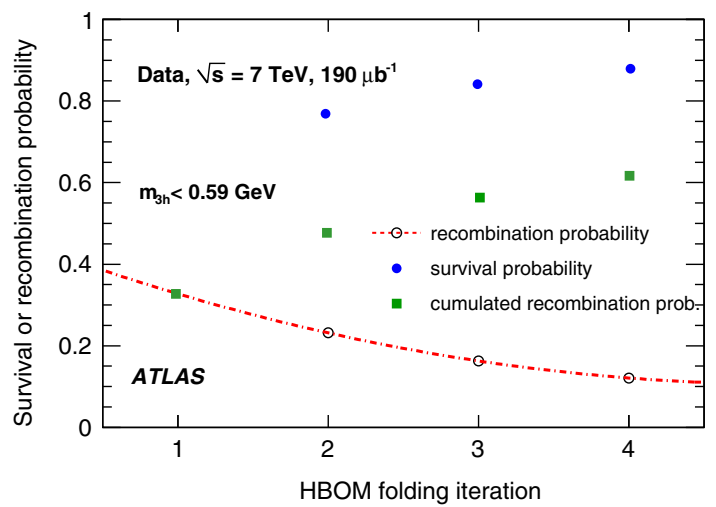

(a)

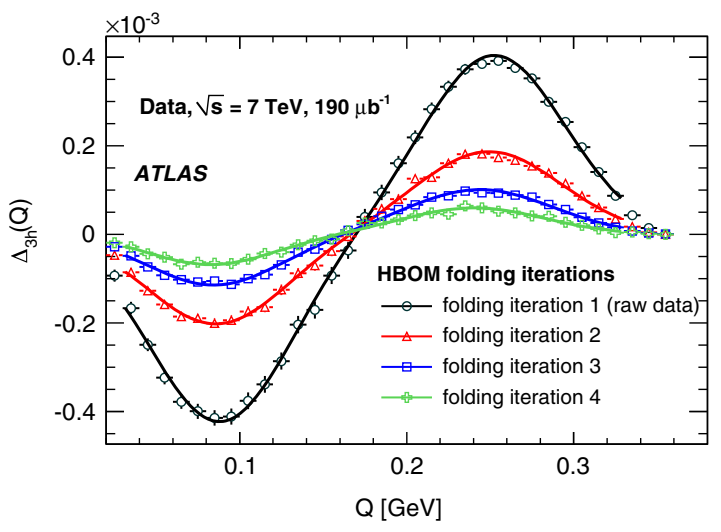

(b)

FIG. 5. Demonstration of the HBOM unfolding procedure. (a) Unfolding fit of the chain recombination probability, used to correct the recombination effects in the detector-level chain selection. The cumulated recombination probability corresponds to the fraction of "nonoriginal" chains - those not existing at the particle level—in each HBOM iteration. (b) $\Delta_{3 h}$ per HBOM iteration, for a chain mass limit of $0.59 \mathrm{GeV}$, fitted with a double-Gaussian parametrization. Both plots show statistical errors only.

correspond to the width of peaks, for like-sign (oppositesign) pairs.

The shape is fitted for four folding iterations (including the raw data), and the fit parameters and correlations between them are unfolded using the HBOM technique. Figure 5(b) shows the fits of the $\Delta_{3 h}(Q)$ distribution for the $m_{3 h}<0.59 \mathrm{GeV}$, in the fit range $Q \in(0.03,0.33) \mathrm{GeV}$. The bin errors of fitted distributions are statistical only.

Figure 6 shows the HBOM unfolding extrapolation of the fit parameters: the position and the width of the negative [like-sign pair (LS)] peak and the position and the width of the positive [opposite-sign pair (OS)] peak. The stability of the unfolding fit is evaluated using a fit with a polynomial
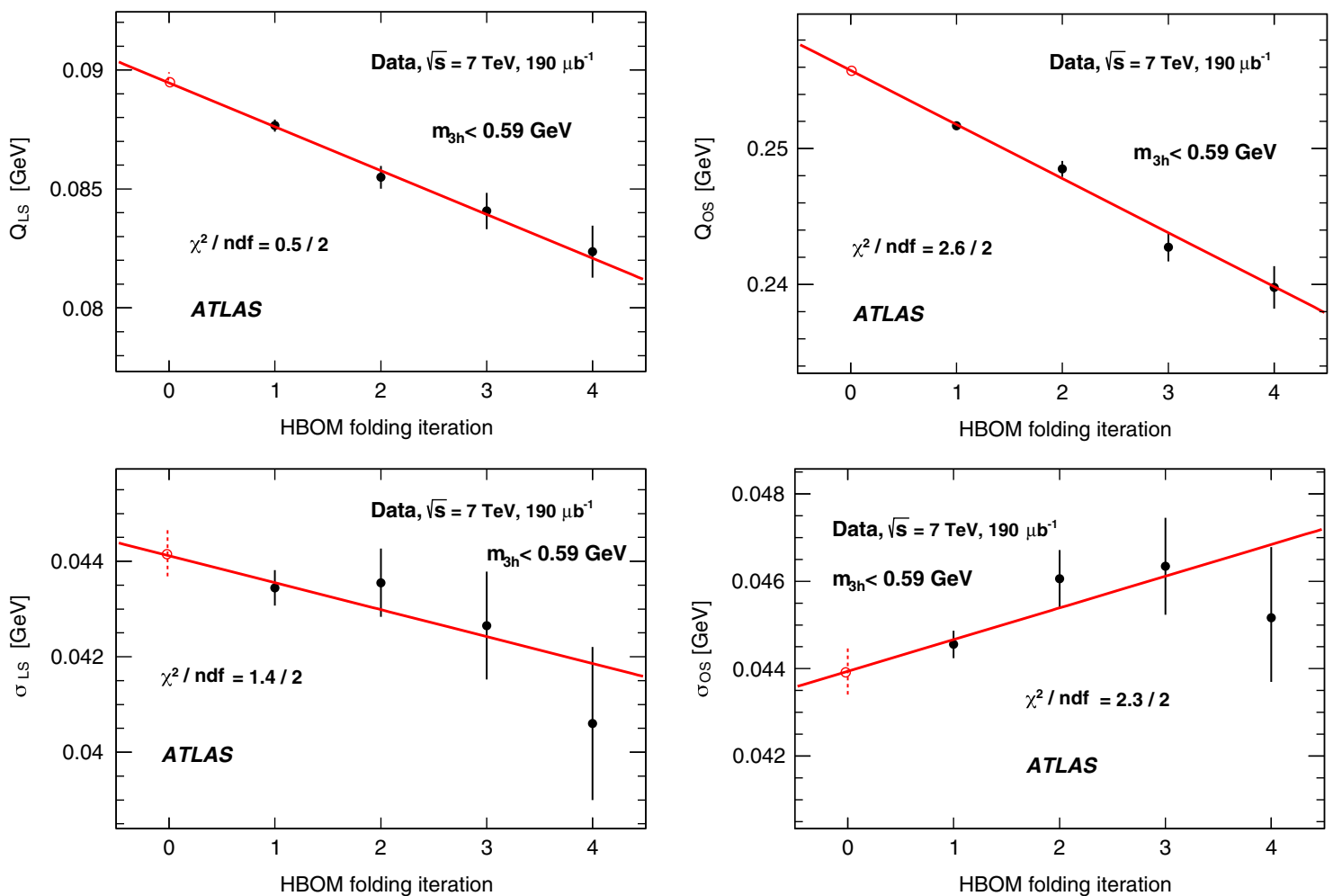

FIG. 6. The unfolding of the peak position (top) and of the Gaussian width (bottom), for the like-sign pairs (left) and opposite-sign pairs (right), for the chain mass limit of $0.59 \mathrm{GeV}$, using the statistical errors only. The black closed points indicate the values of the fit parameter obtained from the fit of $\Delta_{3 h}$, and the open red point is the unfolded value obtained from the HBOM fit. 
TABLE II. The unfolded relative correlation strength CCS/CS for three values of the upper limit on the mass of the triplet chain (column 2). Interpolating between the observed values of CCS/CS, the value of $m_{3 h}^{\text {cut }}$ is adjusted to yield CCS/CS $=1$ (column 3 ). The systematic uncertainty combines reconstruction uncertainty and the uncertainty of folding factors.

\begin{tabular}{|c|c|c|c|c|}
\hline \multirow[b]{2}{*}{ Parameter $(\mathrm{MeV})$} & \multicolumn{3}{|c|}{$m_{3 h}^{\text {cut }}$ (input) } & \multirow[b]{2}{*}{ Interpolation } \\
\hline & 580 & 590 & 600 & \\
\hline $\begin{array}{l}\mathrm{CCS} / \mathrm{CS} \pm \sigma(\text { stat }) \\
m_{3 h}^{\text {cut }} \text { adjusted }\end{array}$ & $0.88 \pm 0.02$ & $0.99 \pm 0.02$ & $1.09 \pm 0.02$ & $\begin{array}{c}1.00 \pm 0.02(\text { stat }) \pm 0.07(\text { syst }) \\
591 \pm 2(\text { stat }) \pm 7(\text { syst })\end{array}$ \\
\hline
\end{tabular}

of higher order and by varying the range of the fit of the $\Delta_{3 h}(Q)$ distribution. Good stability is observed in the unfolding fit of the Gaussian functions mean and width values, the results of the fit variations are compatible within the statistical errors, and therefore no additional systematic uncertainty is attributed to the fit procedure. It is not necessary to unfold the normalization parameters via the HBOM method since they are fixed with much higher precision by the adjustment of the correlation strength. The correlation coefficient is $-0.3(0.5)$ for fitted $Q_{\mathrm{LS}}$ and $Q_{\mathrm{OS}}$ $\left(\sigma_{\mathrm{LS}}\right.$ and $\left.\sigma_{\mathrm{OS}}\right)$ values.

\section{RESULTS}

The value of the upper limit on the chain mass for which the chain selection reproduces the inclusive correlation strength is obtained by interpolation between HBOM results obtained by variation of the chain mass limit $m_{3 h}^{\text {cut }}$ by $10 \mathrm{MeV}$ (Table II).

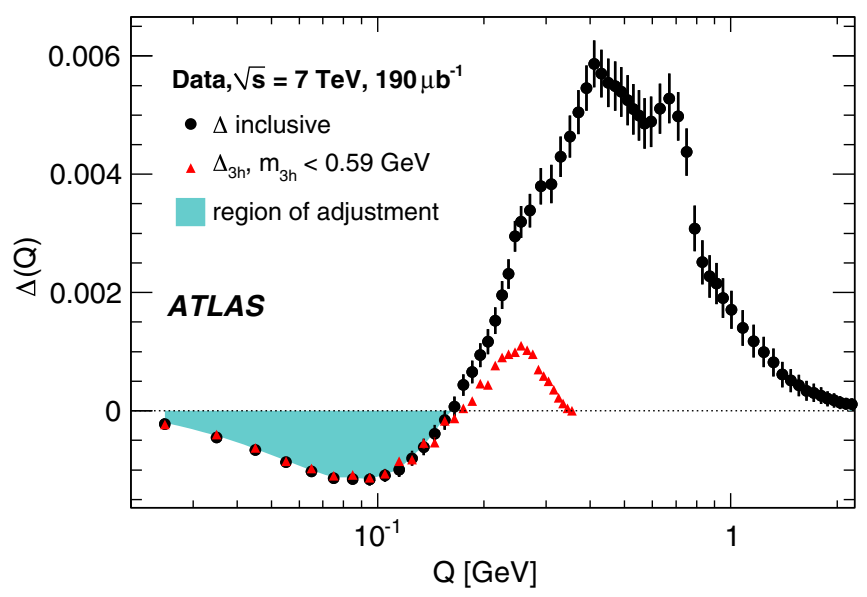

FIG. 7. The corrected $\Delta(Q)$ is compared with the corrected contribution from low-mass three-hadron chains $\Delta_{3 h}(Q)$. The chain mass limit is set to a value of $m_{3 h}^{\text {cut }}=0.59 \mathrm{GeV}$, which reproduces the excess in the inclusive like-sign pair production at low $Q$ (shaded area). Bin errors indicate the combined statistical and reconstruction uncertainty. The uncertainty of the track-based weights is absorbed in the adjustment procedure and translated into uncertainty of the upper chain mass limit.
The resulting adjusted chain mass-limit value

$m_{3 h}^{\text {cut }}(\mathrm{CS}=\mathrm{CCS})=591 \pm 2$ (stat) \pm 7 (syst) $\mathrm{MeV}$

is in agreement with the value $m_{n=3}=570 \pm 20 \mathrm{MeV}$ expected by the helix fragmentation model, Eq. (2).

The comparison of the corrected inclusive correlation function $\Delta(Q)$ with the corrected correlation function $\Delta_{3 h}(Q)$ representing the contribution from the chain selection for the adjusted chain mass limit is shown in Fig. 7. The region of adjustment, indicated by the shaded area, corresponds to the region where the enhanced like-sign pair production is observed.

In order to measure the shape of the correlation function that corresponds to the adjusted chain mass limit, the unfolding of the parametrized shape of $\Delta_{3 h}(Q)$ is repeated for several chain mass limit values $(0.58,0.59$, and $0.6 \mathrm{GeV})$, and the unfolded parameters are interpolated to the adjusted chains mass-limit value (Table III). The reconstruction systematic uncertainty is evaluated by applying a correlated smearing of input bin values according to the pairreconstruction systematic uncertainty. The variation of folding factors has a negligible impact on the shape of the distribution.

The unfolded $\Delta_{3 h}$ measured in the chain selection with the adjusted chain mass limit reproduces the shape of the inclusive correlation function in the low- $Q$ region. In addition, good agreement is observed between the measurement and the helix model predictions for a chain of three ground-state pions (last column in Table III). The width of the peaks is not predicted by the model, although it can be assimilated with the fluctuations of the helix shape of the field. The experimental resolution is better than $10 \mathrm{MeV}$ in the low- $Q$ region.

\section{A. Stability of results}

The analysis was repeated using a single-Gaussian parametrization, with independent fits of the negative and positive regions of $\Delta_{3 h}(Q)$. The change of the fitting function had no significant impact on the results. In addition, the analysis was repeated with HBOM folding accompanied by smearing of reconstructed track parameters according to the reconstruction uncertainty. No significant change in the results was observed. 
TABLE III. Unfolded double-Gaussian parametrization of $\Delta_{3 h}$ for a $10 \mathrm{MeV}$ scan of the chain mass limit and interpolation to the best estimate of $m_{3 h}^{\text {cut }}$ [see also Eq. (10)] compared to the helix model predictions [2]. The systematic uncertainty accounts for all reconstruction effects except the uncertainty associated with track-based correction factors, which serves as input for the evaluation of the chains selection uncertainty (Table IV).

\begin{tabular}{|c|c|c|c|c|c|}
\hline \multirow[b]{2}{*}{ Parameter } & \multicolumn{4}{|c|}{$m_{3 h}^{\text {cut }}(\mathrm{MeV})$} & \multirow[b]{2}{*}{ QCD helix model predictions $(\mathrm{MeV})$} \\
\hline & 580 & $\begin{array}{c}590 \\
\pm \sigma(\text { stat })\end{array}$ & 600 & $\begin{array}{l}591 \text { (best estimate) } \\
\pm \sigma \text { (stat) } \pm \sigma \text { (rec) }\end{array}$ & \\
\hline$Q_{\mathrm{LS}}$ & $86.6 \pm 0.4$ & $89.4 \pm 0.4$ & $92.2 \pm 0.4$ & $89.7 \pm 0.4 \pm 1.2$ & $91 \pm 3$ \\
\hline$\sigma_{\mathrm{LS}}$ & $41.4 \pm 0.6$ & $44.1 \pm 0.6$ & $46.5 \pm 0.7$ & $44.3 \pm 0.6 \pm 2.0$ & $\ldots$ \\
\hline$Q_{\mathrm{OS}}$ & $248.3 \pm 0.5$ & $255.8 \pm 0.5$ & $262.9 \pm 0.5$ & $256.4 \pm 0.5 \pm 1.8$ & $266 \pm 8$ \\
\hline$\sigma_{\mathrm{OS}}$ & $40.9 \pm 0.5$ & $43.9 \pm 0.6$ & $46.5 \pm 0.7$ & $44.2 \pm 0.6 \pm 1.5$ & $\cdots$ \\
\hline
\end{tabular}

The stability of the results was verified by a variation of the acceptance requirements; the pseudorapidity range was reduced from $|\eta|<2.5$ to $|\eta|<1$, and in a separate study, the transverse momentum threshold was raised from 100 to $200 \mathrm{MeV}$. The study of the impact of track migration across the acceptance boundary was performed; a three-hadron chain containing a particle beyond the acceptance boundary was not reconstructed, but the remaining pair contributed to the inclusive spectrum. The fraction of lost chains was traced and found to be $17 \%$ across the pseudorapidity limit at $|\eta|=1$ and $30 \%$ across the transverse momentum limit at $p_{\mathrm{T}}=200 \mathrm{MeV}$. Taking into account the track migration, no significant differences were observed in the correlation shape $\Delta_{3 h}$ measured within restricted acceptance regions.

The loss of chains due to a track beyond the detector acceptance as well as the efficiency of the chain selection cannot be fully assessed by the analysis, and the measured chain mass limit is to be considered as an upper limit only. An estimate of the mass-limit range can be made in the reconstructed data from the rate of like-sign pairs at low $Q$ not associated with the chain selection $(\sim 30 \%)$. Under the hypothesis that one-third of the unassociated like-sign pairs belongs to nonreconstructed chains with very low mass (10\%), the chain mass limit would have to be decreased by $\sim 10 \mathrm{MeV}$ to keep CS = CCS (Table II). Thus, an asymmetric error of $-10 \mathrm{MeV}$ is added and propagated to the systematic uncertainty of the $\Delta_{3 h}(Q)$ parametrization. Table IV provides an overview of systematic uncertainties related to the chain selection.

The variation of the acceptance requirements is also used to examine the variation of the correlation strength with charged-particle multiplicity in the inclusive sample, independently of the chain selection. Table V summarizes the results. The systematic uncertainty is a combination of the uncertainty of the folding factors (5\%) and the bias due to the nonzero total charge in a reconstructed event $(15 \%)$, the latter being evaluated with help of a subsample of events with balanced content of positively and negatively charged tracks. The correlation strength remains stable within the restricted pseudorapidity region, despite the sharp drop of the average charged-particle multiplicity (factor 0.33). Such a behavior supports the hypothesis of a linear dependence of correlations on the number of particles. The measured correlation strength corresponds to the

TABLE IV. Overview of systematic uncertainties derived from the variation of the chain selection.

\begin{tabular}{|c|c|c|c|c|c|c|}
\hline \multirow[b]{2}{*}{ Measured parameter } & \multirow[b]{2}{*}{ Central value $(\mathrm{MeV})$} & \multicolumn{5}{|c|}{ Systematic uncertainty (by source) $(\mathrm{MeV})$} \\
\hline & & Stat & Reconstruction & Unfolding & Acceptance & Combined \\
\hline$m_{3 h}^{\text {cut }}$ & 591 & \pm 2 & \pm 6 & \pm 4 & -10 & $+7.5 /-13$ \\
\hline$Q_{\mathrm{LS}}$ & 89.7 & \pm 2.1 & & & -2.8 & $+2.1 /-3.3$ \\
\hline$\sigma_{\mathrm{LS}}$ & 44.3 & \pm 0.8 & & & -1.0 & $+0.8 /-1.3$ \\
\hline$Q_{\mathrm{OS}}$ & 256.4 & \pm 5.5 & & & -7.3 & $+5.5 /-9.1$ \\
\hline$\sigma_{\mathrm{OS}}$ & 44.2 & \pm 1.9 & & & -2.6 & $+1.9 /-3.2$ \\
\hline
\end{tabular}

TABLE V. Variation of the charged-particle multiplicity and of the correlation strength with the change of acceptance region. $N_{\mathrm{ch}}^{\text {main }}$ stands for the corrected charged-particle multiplicity in the acceptance region of the base analysis. All numbers are corrected for detector effects.

\begin{tabular}{lccc}
\hline \hline Acceptance variations & $p_{\mathrm{T}}>100 \mathrm{MeV}|\eta|<2.5$ & $p_{\mathrm{T}}>100 \mathrm{MeV}|\eta|<1$ & $p_{\mathrm{T}}>200 \mathrm{MeV}|\eta|<2.5$ \\
\hline$N_{\mathrm{ch}} / N_{\mathrm{ch}}^{\text {main }}$ & 1 (by construction) & 0.33 & 0.78 \\
$-\int_{\Delta Q<0} d \Delta Q[\%]$ & $1.07 \pm 0.03$ (stat) ${ }_{-0.17}^{+0.05}($ syst $)$ & $1.24 \pm 0.07(\text { stat })_{-0.21}^{+0.06}($ syst $)$ & $0.56 \pm 0.03$ (stat) $)_{-0.10}^{+0.03}($ syst $)$ \\
\hline \hline
\end{tabular}




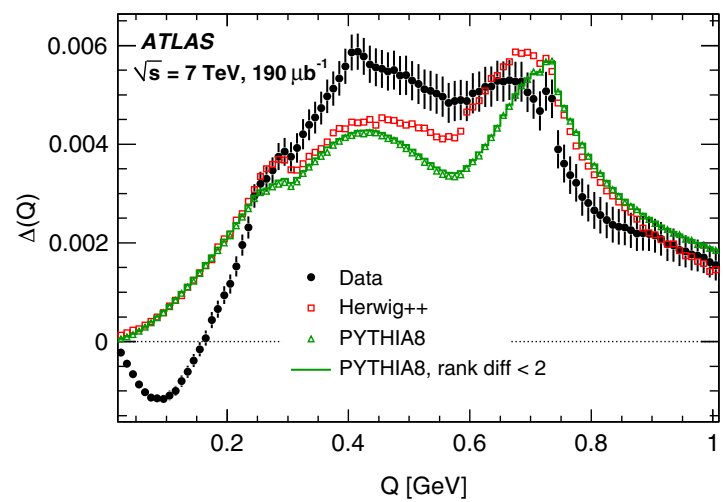

(a)

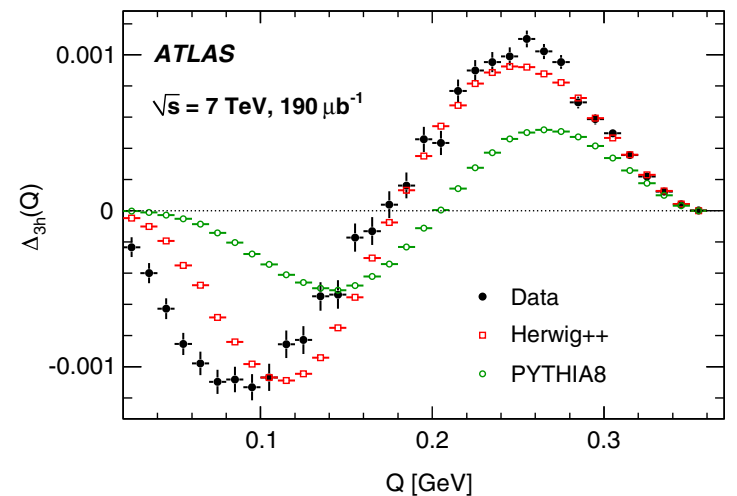

(b)

FIG. 8. (a) The comparison between the measured inclusive $\Delta(Q)$ and the prediction of the Lund string fragmentation model (represented by PYTHIA) and of the cluster hadronization model (represented by HERWIG++). In the Lund string fragmentation model, the inclusive $\Delta$ distribution reflects the distribution of true adjacent hadrons pairs (rank difference $<2$ ), indicated by the full line. (b) Comparison of the measured $\Delta_{3 h}(Q)$ correlation function with a representative set of hadronization models, for the three-hadron chain selection with mass $m_{3 h}<0.59 \mathrm{GeV}$.

minimum rate of three-hadron chains per charged particle required to reproduce the correlation shape of the inclusive $\Delta(Q)$. The observed correlation strength in the baseline acceptance region is rather low, $1.1_{-0.2}^{+0.1} \%$, as reported in Table V. This suggests that it is sufficient to have a single three-pion chain in the ground state per three events to generate enough correlations to reproduce the data.

The correlation strength is reduced by a factor of 2 with the increase of the transverse momentum threshold to $200 \mathrm{MeV}$. In the minimum-bias sample, strings are oriented predominantly along the beam axis, and therefore the observed variation of the correlation strength suggests the correlated hadrons have small intrinsic $p_{\mathrm{T}}$. The quantized fragmentation model predicts the correlations occur between pions with an intrinsic transverse momentum of $\sim 134 \mathrm{MeV}$, with respect to the string axis. The detailed verification of the low- $p_{\mathrm{T}}$ dependence of the chain properties is a challenging task for future studies.

\section{B. Comparison with MC models}

The comparison of the unfolded $\Delta(Q)$ distribution with the predictions of PYTHIA and HERWIG++ is shown in Fig. 8(a). The Lund fragmentation model and the cluster hadronization model predict a very similar distribution in the region of interest for the current analysis $(Q \leq 0.3)$, and both disagree with the data significantly. There is a close correspondence between the inclusive $\Delta(Q)$ observable and the distribution of the momentum difference between true adjacent pairs produced by the string fragmentation, retrieved from the PYTHIA8 event record. This is a feature discussed in Sec. III B; the inclusive $\Delta(Q)$ is constructed in a way which makes it suitable for the study of adjacent hadron pairs. Since the integral of $\Delta(Q)$ is an invariant, there is a direct relation between the excess of measured hadron pairs in the region of $0.3<Q<0.6 \mathrm{GeV}$, with respect to the MC models, and the depletion at $Q<0.2 \mathrm{GeV}$. The hypothesis of a physics threshold in the emission of adjacent hadrons offers a plausible explanation of the discrepancy between the data and the models. According to the MC estimates, the heavy flavor contribution to the low- $Q$ spectrum is negligible.

Figure 8(b) shows the comparison of the measured $\Delta_{3 h}$ with PYTHIA and HERWIG++ predictions. PYTHIA does not describe the data, and the study of the event record reveals that the shape of its prediction is dominated by chains formed by a pair of adjacent hadrons (with opposite charges) and a hadron originating from another string. HERWIG++ describes the chain selection much better even though it fails to reproduce the low- $Q$ part of the measured distribution. A similar observation was made in the study of the azimuthal ordering [6], where some observables were better described by HERWIG++.

Given that neither of these event generators contains the correlation effects induced by the fragmentation of the helical QCD string, yet the data are to some extent described, the presence of chains of ground-state hadrons cannot be assessed from the shape of the measured $\Delta_{3 h}$ alone. A further study of the properties of the selected hadron triplets is discussed in the next section.

\section{Three-body decay}

The selected three-hadron chains with mass below $0.59 \mathrm{GeV}$ are used to fill a Dalitz plot with coordinates $(X, Y)$ defined in Eq. (6). The measurement has the advantage of providing a single entry for each selected hadron triplet (instead of three separate entries in $\Delta_{3 h}$ ) and therefore provides more direct information than the measurement of the correlation shape. However, the combinatorial background is no longer subtracted, which means the correlations are studied on top of a large background distribution. 

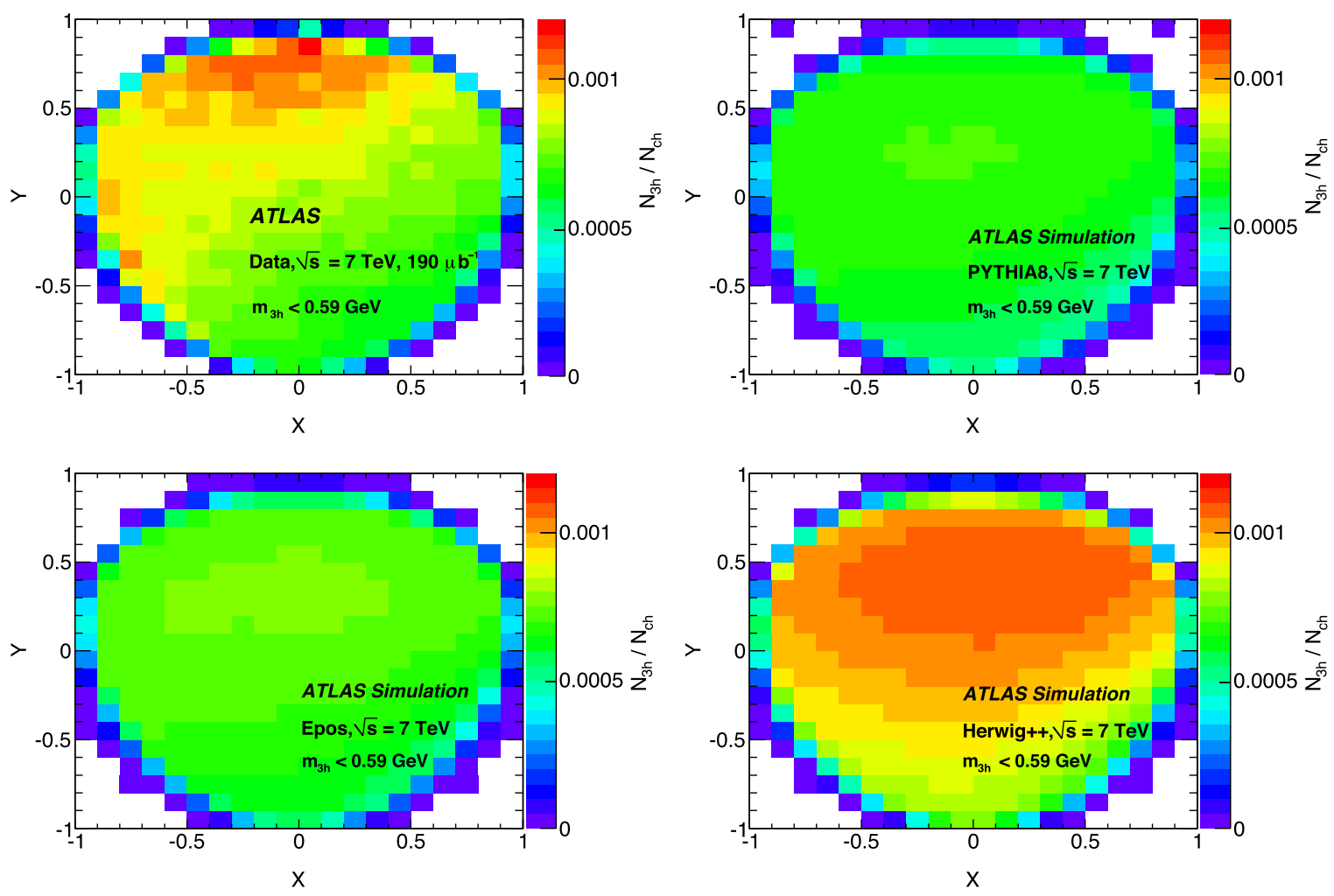

FIG. 9. The Dalitz plot, Eq. (6), filled with the three-body decay pattern of the three-hadron chain selection $\left(m_{3 h}<0.59 \mathrm{GeV}\right)$. Top left: ATLAS data with detector effects unfolded. Top right: PYTHIA8 4C prediction for minimum-bias events. Bottom left: EPOS prediction for minimum-bias events. Bottom right: HERWIG++ prediction for minimum-bias events.

The data are corrected with help of the HBOM estimate of the chain recombination and of the trackbased weight factors as described in Sec. VII. The correction procedure was verified with the PYTHIA6 sample. Figure 9 shows the corrected decay pattern of selected chains in the data and the equivalent obtained from the generator-level MC samples. The distributions are normalized to the number of charged particles in the sample.
The rate of selected chains is $0.24 \pm 0.02$ per charged particle in the data, 0.18 in PYTHIA8, 0.20 in EPOS, and 0.28 in HeRwiG++. A clear enhancement is observed in the data at large $Y$ values, which is not reproduced by any of the MC samples (the quantum effects affecting the hadron momenta are not included in the event generators).

In order to quantify the significance of the shape difference between the data and the MC models, the Dalitz plot is split into three regions; see Fig. 10. The
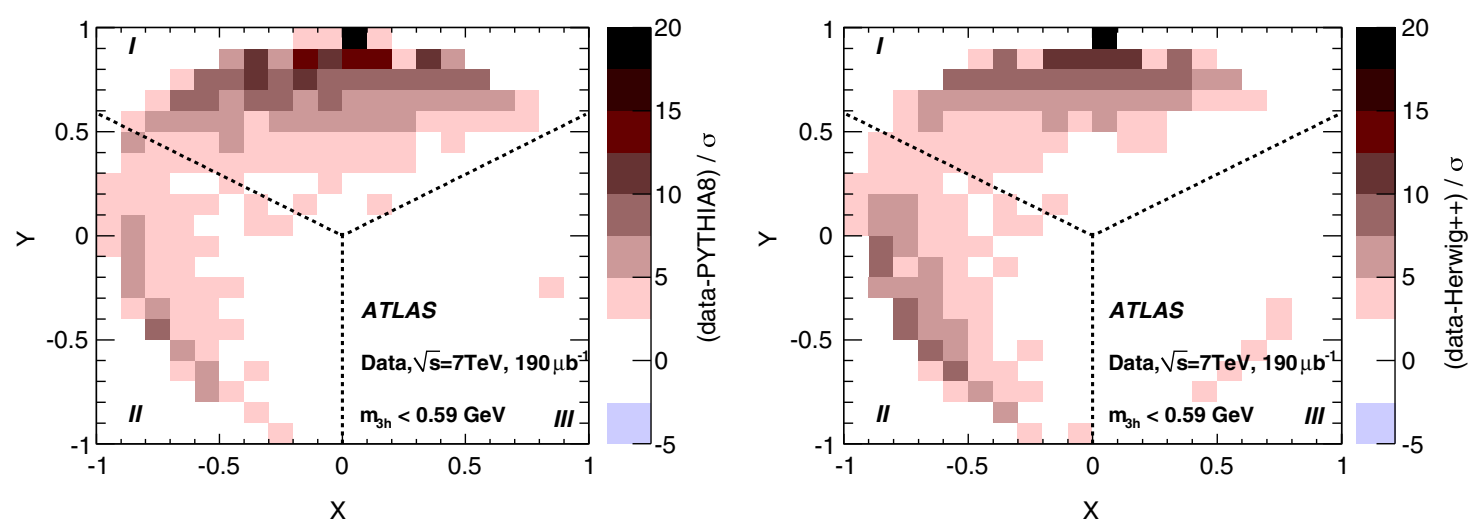

FIG. 10. The significance of the difference of the Dalitz plot filled with the three-body decay pattern of chains with mass below $0.59 \mathrm{GeV}$, between the data and the PYTHIA8 simulation (left) and the HERWIG++ simulation (right). The signal region I gathers chains where the pair of like-sign hadrons carries the least momentum difference. Region III serves as a reference for the adjustment of the MC simulation with respect to the data. 
principal signal region I gathers chains where the pair of like-sign hadrons carries the smallest momentum difference. A less pronounced but still quite significant excess is observed in region II where it can be interpreted as a signature of a quadruplet chain containing a neutral pion (see below). The models are rescaled to reproduce the rate of selected chains in the reference region III where no enhancement is observed and where no enhancement is expected by the quantized helix string fragmentation model (see Sec. IX). The significance of the difference between the data and the MC prediction is then evaluated as the number of standard deviations observed, calculated from the statistical error and from the uncertainty associated with the bin migration and with the $X, Y$ dependence of trackbased weights and of the presence of nonprimary tracks. The reconstruction uncertainty as well as the correlated uncertainty of folding/weighting factors are strongly correlated between bins and therefore absorbed in the adjustment of the MC chain rate. The $\chi^{2}$ per degree of freedom for comparison of data with PYTHIA8 is 29.1, 8.1, and 0.89 in regions I, II, and III, respectively. The comparison of data with HERWIG++ yields similar results: $\chi^{2}$ per degree of freedom $=20.0,12.4$, and 0.98 in regions I, II, and III, respectively. The excess in region I, which contains triplets where the like-sign pair of particles carries the smallest momentum difference is $1.72 \pm 0.05 \%(1.35 \pm 0.05 \%)$ of chains per charged particle compared to the adjusted PYTHIA8 (HeRWIG++) shape. The excess in region II is $0.75 \pm 0.04 \%(0.97 \pm 0.04 \%)$ of chains per charged particle compared to the PYTHIA8 (HERWIG++) shape.

\section{INTERPRETATION OF MEASUREMENTS USING THE HELIX STRING MODEL}

The observations described in the previous section agree with the hypothesis that the observed correlations reflect the pattern of the coherent emission of hadrons from a helical QCD string. Since the adjusted chain mass limit agrees well with the expected minimum mass for a true chain of charged pions, the position of the maximum of the $\Delta_{3 h}(Q)$ distribution can be interpreted as the measurement of the momentum difference between adjacent oppositesign pairs of pions with rank difference 1, while the position of the minimum can be associated with the momentum difference between like-sign pairs of pions with rank difference 2 .

\section{A. Onset of adjacent hadron pair production}

The existence of a quantum threshold for the minimum momentum difference between adjacent hadrons is a fundamental feature of the quantized fragmentation model. The data are in agreement with the prediction of a thresholdlike behavior: after the subtraction of selected threehadron chains from the inclusive $\Delta(Q)$, there are no adjacent pairs visible in the low-Q region, up to a certain

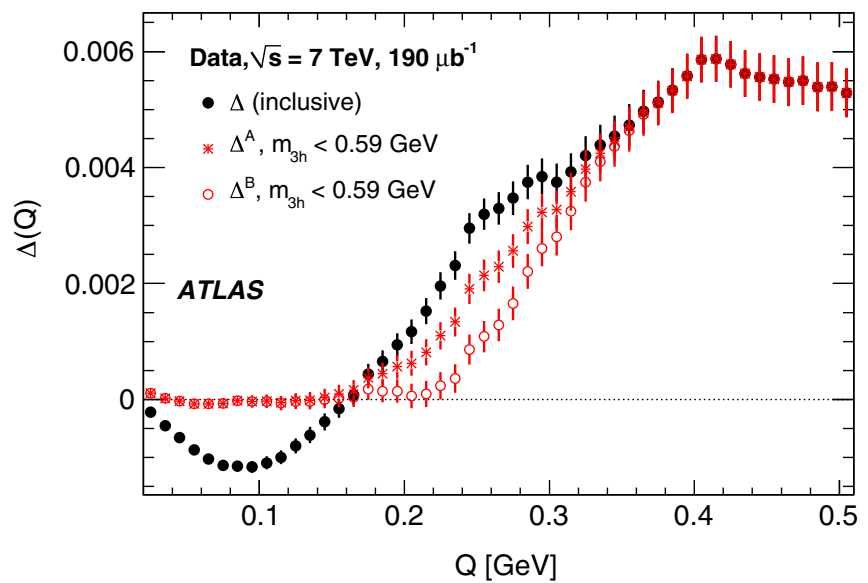

FIG. 11. The unfolded $\Delta(Q)$ with (black points) and without (red points) the contribution from low-mass three-hadron chains. Closed squares indicate the subtraction performed assuming long uninterrupted chains (A), and open circles indicate the subtraction done assuming disconnected triplets (B).

threshold value, which depends on the assumption made about the length of correlated hadron chains:

$$
\begin{gathered}
\Delta^{\mathrm{A}}(Q)=\Delta(Q)-\Delta_{3 h}(Q), \\
\Delta^{\mathrm{B}}(Q)=\Delta(Q)-\Delta_{3 h}(Q)-f_{\mathrm{OS}}\left(Q ; Q_{\mathrm{OS}}, \sigma_{\mathrm{OS}}\right) .
\end{gathered}
$$

Hypotheses A and B refer to Fig. 2. Hypothesis A describes the chain contribution as a contribution from long uninterrupted chains. Hypothesis B assumes the triplet chains are disconnected and restores the contribution from opposite-sign pairs, which had been scaled by factor 0.5 in Eq. (5), using the fitted decomposition of $\Delta_{3 h}$ into two Gaussian functions, Eq. (9).

The distributions after the subtractions are shown in Fig. 11. In the scenario of disconnected three-pion chains, the threshold value moves up to $\sim 0.25 \mathrm{GeV}$. This value coincides with the quantum threshold predicted by the quantized model of fragmentation of a helical QCD string, which also fits the experimentally found position of the peak formed by closest opposite-sign pairs.

\section{B. Enhanced production of pairs of like-sign charged hadrons}

The enhanced production of pairs of like-sign charged hadrons is traditionally attributed to the Bose-Einstein effect, originating in the symmetrization of the quantummechanical amplitude with respect to the exchange of identical bosons [7]. A large number of measurements have been done on the basis of the correlation function defined as a ratio of like-sign and opposite-sign distributions, the latter being considered "uncorrelated" by the Bose-Einstein formalism. 


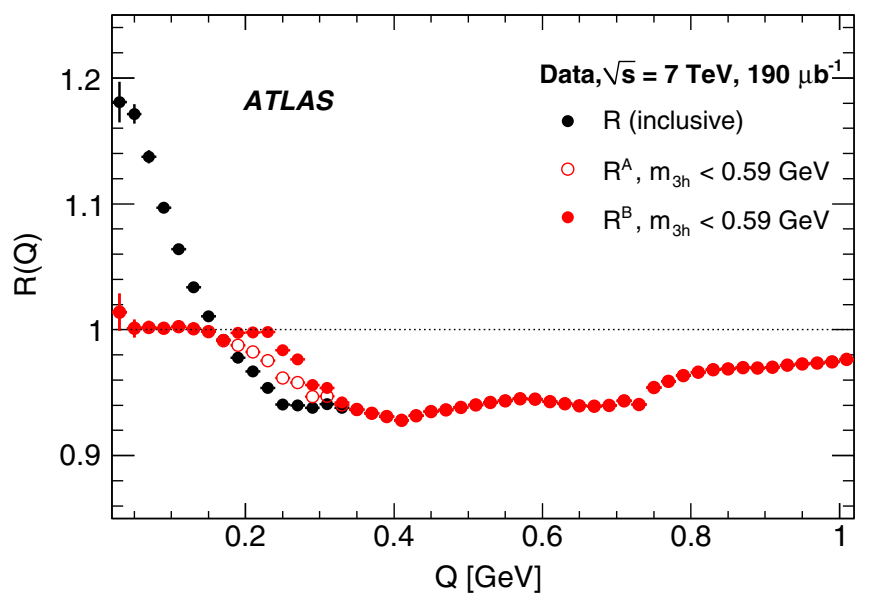

FIG. 12. The ratio of inclusive like-sign and opposite-sign pair spectra, before and after subtraction of the estimated contribution from low-mass three-hadron chains, in scenario A (long uninterrupted chain) and B (disconnected triplet chains). The upper mass limit for the three-hadron chain $(0.59 \mathrm{GeV})$ has been set to the value that reproduces the enhancement of like-sign pair production in the inclusive sample.

Figure 12 shows the ratio $R=N(Q)^{\mathrm{LS}} / N(Q)^{\mathrm{OS}}$ before and after subtraction of the estimated contribution from ordered hadron chains with mass below $0.59 \mathrm{GeV}$. The subtraction is done in close analogy to Eqs. (11) and (12) with the help of the fit [Eq. (9)],

$$
\begin{aligned}
R^{\mathrm{A}}(Q)= & {\left[N(Q)^{\mathrm{LS}}+N_{\mathrm{ch}} f_{\mathrm{LS}}\left(Q ; Q_{\mathrm{LS}}, \sigma_{\mathrm{LS}}\right)\right] / } \\
& {\left[N(Q)^{\mathrm{OS}}-N_{\mathrm{ch}} f_{\mathrm{OS}}\left(Q ; Q_{\mathrm{OS}}, \sigma_{\mathrm{OS}}\right)\right], } \\
R^{\mathrm{B}}(Q)= & {\left[N(Q)^{\mathrm{LS}}+N_{\mathrm{ch}} f_{\mathrm{LS}}\left(Q ; Q_{\mathrm{LS}}, \sigma_{\mathrm{LS}}\right)\right] / } \\
& {\left[N(Q)^{\mathrm{OS}}-2 N_{\mathrm{ch}} f_{\mathrm{OS}}\left(Q ; Q_{\mathrm{OS}}, \sigma_{\mathrm{OS}}\right)\right] ; }
\end{aligned}
$$

i.e. the $\Delta_{3 h}$ distribution is decomposed into contributions from like-sign and opposite-sign pairs, which are subtracted from their respective inclusive two-particle distributions, before calculating the ratio. The subtraction is done for the hypothesis of long chains [A] (open red points) and disconnected chains $[\mathrm{B}]$ (closed red points). In both cases, the chain selection contains the source of the enhanced like-sign pair production, hence providing an alternative explanation of the data.

\section{Three-body decay properties of quantized fragmentation}

Figure 13 compares the measured three-body decay pattern of the chain selection with the model prediction obtained by generating hadron triplets and quadruplets
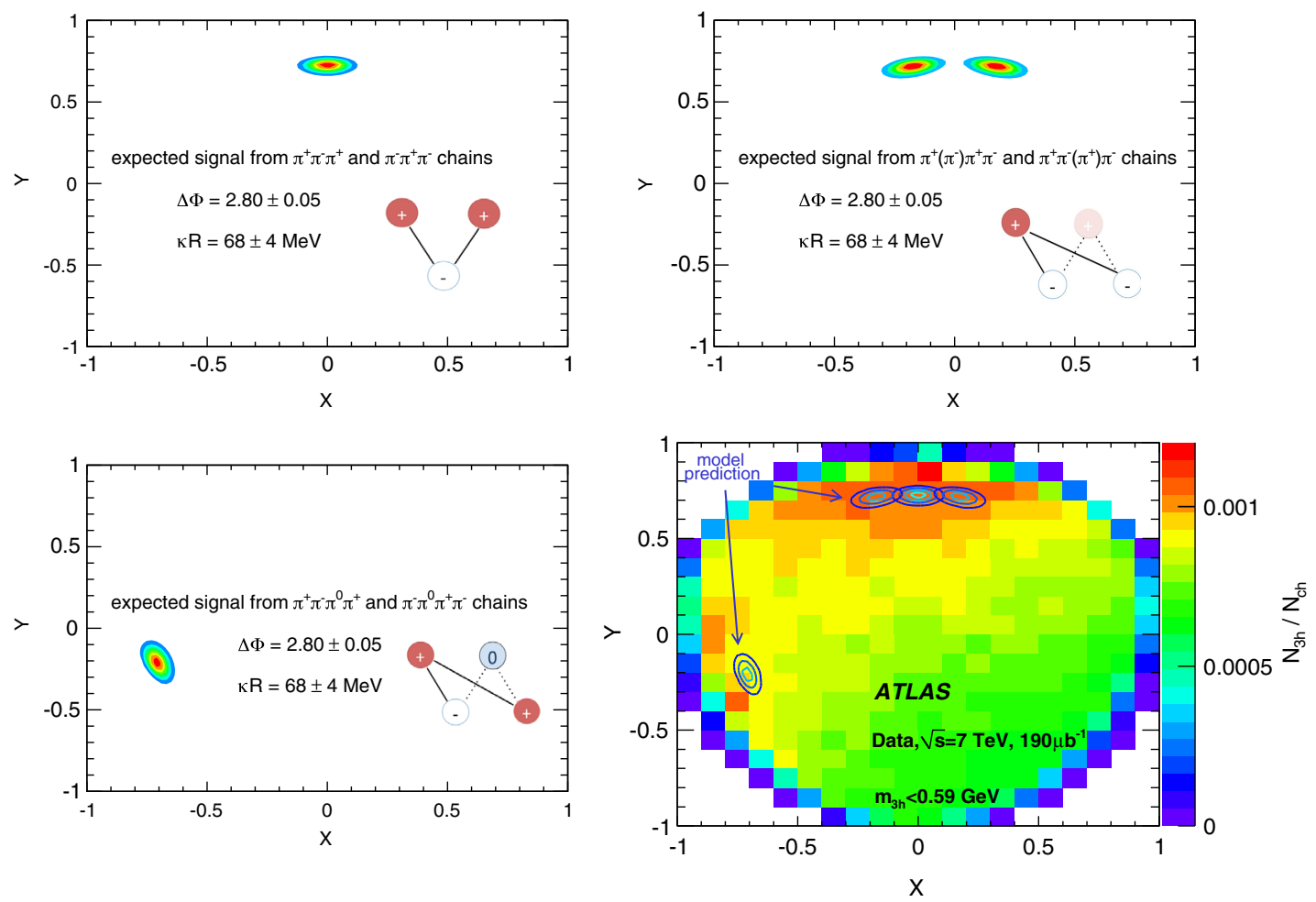

FIG. 13. Model prediction for the decay pattern of the ground-state triplet (top left), ground-state quadruplet with a missing middle member (top right), and ground-state quadruplet containing a neutral pion (bottom left), in an arbitrary z scale. Model predictions are calculated using a Gaussian smearing of helical string parameters constrained by the fit of the mass spectrum of pseudoscalar mesons. The resolution uncertainty of the order of $10 \mathrm{MeV}$ is included in the variation of string parameters. Bottom right: Comparison of the observed chain decay pattern with model predictions. 
according to the pattern of helical string fragmentation into chains of ground-state pions (see the Appendix). String parameters $\Delta \Phi$ and $\kappa R$ are smeared by $2 \%$ and $6 \%$, respectively. The maximal enhancement in the data corresponds to the expected location of the signal from the chain of three ground-state pions, with some indication for the possible presence of quadruplet chains; the wide "shoulder" of the signal in region I can be interpreted as a signature of a quadruplet chain with a missing middle particle. The observation of such a form of signal has implications for the interpretation of the results of the measurements. If there is a significant admixture of opposite-sign pairs with rank difference 3 (for which the momentum difference should be around $0.236 \mathrm{GeV}$ ), the bias should be taken into account in the interpretation of results in terms of properties of the QCD string. This bias is obtained from the best fit of the Dalitz plot with the mixture of the PYTHIA6 shape (for background) and from the model predictions for the triplet and for the incomplete-quadruplet chains (for the signal) and found to be $-9 \pm 5 \mathrm{MeV}$. The uncertainty is estimated as one-third of the case, where the selection is equally spread between pairs with rank differences 1 and 3 .

As described in the Introduction, the quantized helical string fragmentation model draws input from the mass spectrum of light pseudoscalar mesons. It is therefore possible that their decay contributes to the visible correlation pattern, but such a measurement would require the reconstruction of $\pi^{0}$ and therefore falls beyond the scope of the current analysis.

\section{Momentum difference as a function of hadron rank difference}

Taking into account the uncertainty of the chain selection (Table IV), the inclusive two-particle correlation pattern is reproduced by three-hadron chains below a mass limit of

$$
m_{3 h}^{\text {cut }}=591 \pm 2(\text { stat })_{-13}^{+7.5}(\text { syst }) \mathrm{MeV}
$$

The data show a threshold effect in the production of adjacent hadron pairs. The threshold coincides with the emergence of the Gaussian peak situated at

$$
\begin{aligned}
Q_{\mathrm{OS}}= & 256.4 \pm 0.5(\mathrm{stat}) \\
& \pm 1.8(\mathrm{rec})_{-9.1}^{+5.5}(\text { chain selection }) \mathrm{MeV},
\end{aligned}
$$

which is obtained from the fit of the preferred momentum difference between opposite-sign pairs in the selected chains. Taking into account the possible admixture of pairs with rank difference 3 , suggested by the particular form of the three-body decay pattern of selected hadron chains, the best estimate of the momentum difference for hadron pairs of rank difference $r=1$ becomes

$$
\begin{aligned}
Q(r=1)= & 265.6 \pm 0.5(\text { stat }) \\
& \pm 1.8(\mathrm{rec})_{-10}^{+7.4}(\text { chain selection }) \mathrm{MeV} .
\end{aligned}
$$

The preferred momentum difference between hadrons with like-sign charge combination (rank difference $r=2$ ) is found to be

$$
\begin{aligned}
Q(r=2)= & 89.7 \pm 0.4(\text { stat }) \\
& \pm 1.2(\mathrm{rec})_{-3.3}^{+2.1}(\text { chain selection }) \mathrm{MeV}
\end{aligned}
$$

for the same set of selected three-hadron chains. Both likesign and opposite-sign pair distributions have a Gaussian shape with a width of $44 \pm 3 \mathrm{MeV}$, while the experimental resolution in the fitted region is better than $10 \mathrm{MeV}$. The systematic uncertainties in Eqs. (17) and (18) are correlated.

These values are in good agreement with the predictions of the model of a QCD string with a helical shape, with parameters constrained by the mass spectrum of pseudoscalar mesons (see Table I).

\section{CONCLUSIONS}

Two-particle correlation spectra measured in the minimum-bias sample at a center-of-mass energy of $7 \mathrm{TeV}$ are analyzed in the context of coherent particle production. The data sample consists of $190 \mu \mathrm{b}^{-1}$ of events produced with low-luminosity proton-proton beams at the LHC and collected in the early 2010 ATLAS data taking. The QCD string fragmentation scenario is used to introduce the notion of ordered hadron chains. Using the assumption of the local charge conservation in the string breakup, the correlation function is defined in a way suitable for study of correlations between pairs and triplets of adjacent hadrons. Because it is experimentally impossible to assess the exact rank ordering of particles, the rank ordering is replaced by the minimization of the mass of hadron chains. The analysis relies on the removal of the background of random combinations by means of the subtraction of pairs with like-sign charge combination from pairs with opposite-sign charge combination. The analysis does not rely on predictions of conventional MC models which fail to describe the data.

The results indicate that the enhanced like-sign pair production at low $Q$, observed in the data and traditionally attributed to the Bose-Einstein effect, can be entirely attributed to the presence of ordered three-hadron chains with mass below $591_{-13}^{+8} \mathrm{MeV}$, at a minimum rate of $1.1_{-0.2}^{+0.1} \%$ per charged particle. A strong dependence of the size of the effect on the transverse momentum of tracks in the laboratory frame is observed.

The shape of the three-hadron chain contribution to the inclusive $Q$ spectra agrees with the hypothesis that these chains are produced via coherent quantized fragmentation of a homogeneous QCD string with a helical structure. 
The measured momentum difference between hadrons within such a chain is $89.7_{-3.5}^{+2.5} \mathrm{MeV}$ for pairs with rank difference 2 and $266_{-11}^{+8} \mathrm{MeV}$ for pairs of adjacent hadrons. The data support the prediction of a "forbidden" region for the production of adjacent (opposite-sign) hadron pairs at low $Q$. The threshold is situated at $\sim 0.25 \mathrm{GeV}$ and agrees with the quantum threshold predicted by the helical string model.

\section{ACKNOWLEDGMENTS}

We thank CERN for the very successful operation of the LHC as well as the support staff from our institutions, without whom ATLAS could not be operated efficiently. We acknowledge the support of ANPCyT, Argentina; YerPhI, Armenia; ARC, Australia; BMWFW and FWF, Austria; ANAS, Azerbaijan; SSTC, Belarus; CNPq and FAPESP, Brazil; NSERC, NRC, and CFI, Canada; CERN; CONICYT, Chile; CAS, MOST, and NSFC, China; COLCIENCIAS, Colombia; MSMT CR, MPO CR, and VSC CR, Czech Republic; DNRF and DNSRC, Denmark; IN2P3-CNRS, CEA-DSM/IRFU, France; SRNSF, Georgia; BMBF, HGF, and MPG, Germany; GSRT, Greece; RGC, Hong Kong SAR, China; ISF, ICORE, and Benoziyo Center, Israel; INFN, Italy; MEXT and JSPS, Japan; CNRST, Morocco; NWO, Netherlands; RCN, Norway; MNiSW and NCN, Poland; FCT, Portugal; MNE/IFA, Romania; MES of Russia and NRC KI, Russian Federation; JINR; MESTD, Serbia; MSSR, Slovakia; ARRS and MIZŠ, Slovenia; DST/NRF, South Africa; MINECO, Spain; SRC and Wallenberg Foundation, Sweden; SERI, SNSF, and Cantons of Bern and Geneva, Switzerland; MOST, Taiwan; TAEK, Turkey; STFC, United Kingdom; and DOE and NSF, U.S.. In addition, individual groups and members have received support from BCKDF, the Canada Council, CANARIE, CRC, Compute Canada, FQRNT, and the Ontario Innovation Trust, Canada; EPLANET, ERC, ERDF, FP7, Horizon 2020, and Marie SkłodowskaCurie Actions, European Union; Investissements d'Avenir Labex and Idex, ANR, Région Auvergne and Fondation Partager le Savoir, France; DFG and AvH Foundation, Germany; Herakleitos, Thales and Aristeia programs cofinanced by EU-ESF and the Greek NSRF; BSF, GIF and Minerva, Israel; BRF, Norway; CERCA Programme Generalitat de Catalunya, Generalitat Valenciana, Spain; and the Royal Society and Leverhulme Trust, United Kingdom. The crucial computing support from all WLCG partners is acknowledged gratefully, in particular from CERN, the ATLAS Tier-1 facilities at TRIUMF (Canada), NDGF (Denmark, Norway, Sweden), CC-IN2P3 (France), KIT/GridKA (Germany), INFN-CNAF (Italy), NL-T1 (Netherlands), PIC (Spain), ASGC (Taiwan), RAL (United Kingdom) and BNL (U.S.), the Tier-2 facilities worldwide and large
non-WLCG resource providers. Major contributors of computing resources are listed in Ref. [22].

\section{APPENDIX: CALCULATION OF PREDICTIONS WITHIN THE HELICAL QCD STRING MODEL}

In the model described in Ref. [2], the causal constraint applied to the helical QCD field leads to a quantization pattern describing the mass spectrum of hadrons with mass below $1 \mathrm{GeV}$. In particular, the pseudoscalar mesons $\left(\pi, \eta, \eta^{\prime}\right)$ can be regarded as string pieces fragmenting into $(n=1,3,5)$ ground-state hadrons (pions), with transverse energy $\left(E_{\mathrm{T}}\right)$ and momentum $\left(\overrightarrow{p_{\mathrm{T}}}\right)$ of mesons defined by the helical string properties (Fig. 1),

$$
E_{\mathrm{T}}(n)=\sqrt{m_{n}^{2}+p_{\mathrm{T}}(n)^{2}}=n \kappa R \Delta \Phi,
$$

where $R$ stands for the radius of the helix, $\kappa \sim 1 \mathrm{GeV} / \mathrm{fm}$ is the string tension, $\Delta \Phi$ is the quantized helix phase difference describing the shortest piece of string that can form a hadron, and $m_{n}$ is the (quantized) meson mass spectrum.

The transverse momentum of a hadron stems from the integral of the string tension along the trajectory (in the transverse plane) of a quark traveling from one breakup vertex to another. The string tension is tangential to the trajectory of the quark:

$$
\begin{aligned}
& \vec{p}_{\mathrm{T}}(\Phi, \Phi+n \Delta \Phi) \\
& \quad=\left(p_{\mathrm{T}}^{x}, i p_{\mathrm{T}}^{y}\right)=\kappa R \int_{\Phi}^{\Phi+n \Delta \Phi} \mathrm{e}^{\mathrm{i}\left(\alpha+\frac{\pi}{2}\right)} d \alpha \\
& \quad=2 \kappa R \sin \frac{n \Delta \Phi}{2} \mathrm{e}^{\mathrm{i}\left(\Phi+\frac{n \Delta \Phi}{2}+\frac{\pi}{2}\right)} .
\end{aligned}
$$

The fit of the mass spectrum of pseudoscalar mesons indicates a rather narrow radius of the helical string $(\kappa R=68 \pm 2 \mathrm{MeV})$ and a quantized phase difference $\Delta \Phi=2.82 \pm 0.06$. These values translate into the quantized ground-state transverse energy, $E_{\mathrm{T}}(n=1) \simeq 192 \mathrm{MeV}$, and the transverse momentum of a ground-state pion, $p_{\mathrm{T}}(n=1) \simeq 134 \mathrm{MeV}[2]$.

When a piece of helical string fragments into a chain of ground-state pions, the string shape is reflected in the momentum difference of the emitted hadrons. Neglecting the longitudinal momentum differences between adjacent hadrons i.e. assuming local homogeneity of the fragmenting QCD field, the four-momentum difference between hadrons within a chain of ground-state pions can be written as a function of their rank difference $r$,

$Q(r)=\sqrt{-\left(p_{i}-p_{i+r}\right)^{2}}=2 p_{\mathrm{T}}(n=1)|\sin (r \Delta \Phi / 2)|$, 
where $r \Delta \Phi$ corresponds to the opening angle between pions in the transverse plane. Equation (A3) is used to fill Table I.

It is straightforward to calculate the (transverse) momenta of pions in the chain defined by string breakup vertices $\Phi_{j+1}=\Phi_{j}+\Delta \Phi, \ldots, \Phi_{j+n}=\Phi_{j}+n \Delta \Phi$, and to study the properties of pion multiplets for various rank difference combinations. A closer look at Table I suggests the minimization of the mass of selected three-hadron clusters does not ensure the selection of a triplet of adjacent pions in case of the presence of a longer chain. The largest contamination presumably comes from an incomplete quadruplet chain with a missing (internal) pion and from a quadruplet chain containing a neutral pion. Quadruplet chains are therefore included as a correction to the model predictions. For the study of three-body decays, pions in selected triplets are boosted into the rest frame of the triplet, and their kinetic energy is evaluated (the longitudinal momenta of triplet members are negligible in the rest frame of the triplet).

The helix-string model predictions for triplets of charged pions composed of particles with rank difference $r<4$ are shown in Fig. 13, using a numerical smearing of the measured parameters $\kappa R$ and $\Delta \Phi$ (by a Gaussian distribution with a width of $6 \%$ and $2 \%$, respectively). Figure 14 shows the corresponding shapes of the correlation function $\Delta_{3 h}(Q)$. The presence of a pair with rank difference 3 modifies the shape of the positive part of the $\Delta_{3 h}$ distribution. The presence of a neutral pion within the chain

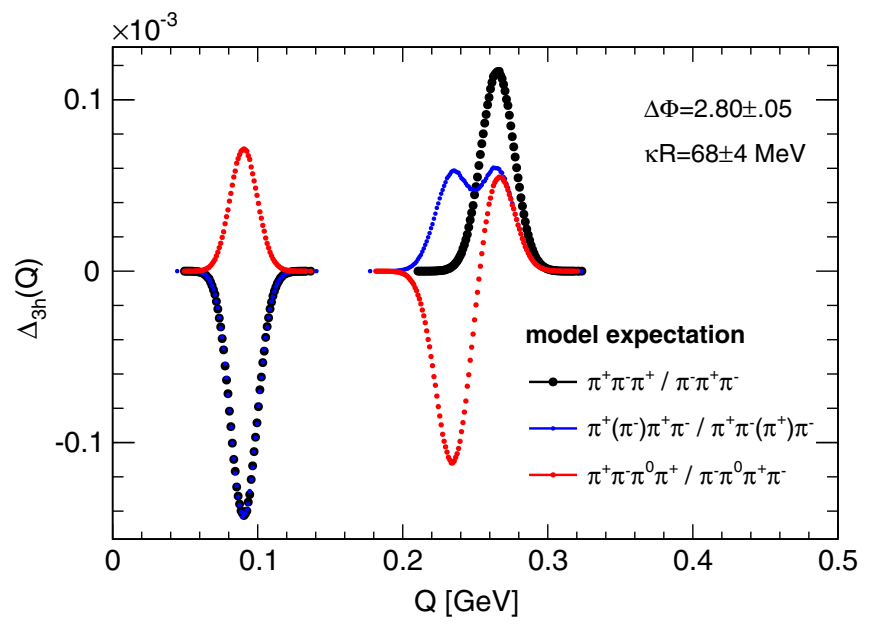

FIG. 14. Evaluation of the model prediction for the shape of the correlation function $\Delta_{3 h}$ produced by the ground-state triplet (black points), ground-state quadruplet with a missing middle member (blue points), and ground-state quadruplet containing a neutral pion (red points), for an equal number of triplets of each type. Model predictions are calculated using a Gaussian smearing of helical string parameters constrained by the fit of the mass spectrum of pseudoscalar mesons. The resolution uncertainty of the order of $10 \mathrm{MeV}$ is included in the variation of string parameters.

tends to diminish the correlation signal by compensating, to some extent, the asymmetry between the like-sign and the opposite-sign pair $Q$ distributions.
[1] ALEPH, DELPHI, L3, OPAL, and LEP Electroweak Working Group Collaborations, Electroweak measurements in electron-positron collisions at W-bosonpair energies at LEP, Phys. Rep. 532, 119 (2013) (Sect. 4.2).

[2] S. Todorova, Quantization of the QCD string with a helical structure, Phys. Rev. D 89, 015002 (2014).

[3] B. Andersson, G. Gustafson, J. Hakkinen, M. Ringner, and P. Sutton, Is there screwiness at the end of the QCD cascades?, J. High Energy Phys. 09 (1998) 014.

[4] B. Andersson, G. Gustafson, G. Ingelman, and T. Sjöstrand, Parton fragmentation and string dynamics, Phys. Rep. 97, 31 (1983).

[5] T. Sjöstrand, S. Mrenna, and P.Z. Skands, A brief introduction to PYTHIA 8.1, Comput. Phys. Commun. 178, 852 (2008).

[6] ATLAS Collaboration, Measurement of the azimuthal ordering of charged hadrons with the ATLAS detector, Phys. Rev. D 86, 052005 (2012).

[7] ATLAS Collaboration, Two-particle Bose-Einstein correlations in $p p$ collisions at $\sqrt{s}=0.9$ and $7 \mathrm{TeV}$ measured with the ATLAS detector, Eur. Phys. J. C 75, 466 (2015).
[8] HepData record of Ref. [7], http://dx.doi.org/10.17182/ hepdata.70016.

[9] F. Ambrosino et al. (KLOE Collaboration), Determination of $\eta \rightarrow \pi^{+} \pi^{-} \pi^{0}$ Dalitz plot slopes and asymmetries with the KLOE detector, J. High Energy Phys. 05 (2008) 006.

[10] P. Adlarson (WASA@COSY Collaboration), Measurement of the $\eta \rightarrow \pi^{+} \pi^{-} \pi^{0}$ Dalitz plot distribution, Phys. Rev. C 90, 045207 (2014).

[11] ATLAS Collaboration, The ATLAS Experiment at the CERN Large Hadron Collider, JINST 3, S08003 (2008).

[12] ATLAS Collaboration, Charged-particle multiplicities in pp interactions measured with the ATLAS detector at the LHC, New J. Phys. 13, 053033 (2011).

[13] T. Sjöstrand, S. Mrenna, and P.Z. Skands, PYTHIA 6.4 physics and manual, J. High Energy Phys. 05 (2006) 026.

[14] ATLAS Collaboration, Report No. ATL-PHYS-PUB-2010002, 2010, https://cds.cern.ch/record/1247375.

[15] S. Agostinelli et al. (GEANT4 Collaboration), GEANT4: A simulation toolkit, Nucl. Instrum. Methods Phys. Res., Sect. A 506, 250 (2003).

[16] ATLAS Collaboration, The ATLAS simulation infrastructure, Eur. Phys. J. C 70, 823 (2010). 
[17] R. Corke and T. Sjöstrand, Interleaved parton showers \& tuning prospects, J. High Energy Phys. 03 (2011) 032.

[18] M. Bahr et al., Herwig++ physics and manual, Eur. Phys. J. C 58, 639 (2008).

[19] S. Gieseke, C. A. Röhr, and A. Siódmok, Multiple partonic interaction developments in Herwig++, arXiv:1110.2675.
[20] S. Porteboeuf et al., The EPOS event generator, arXiv: 1006.2967.

[21] J. W. Monk and C. Oropeza-Barrera, The HBOM method for unfolding detector effects, Nucl. Instrum. Methods Phys. Res., Sect. A 701, 17 (2013).

[22] ATLAS Collaboration, Report No. ATL-GEN-PUB-2016002, 2016, https://cds.cern.ch/record/2202407.

M. Aaboud, ${ }^{137 d}$ G. Aad,${ }^{88}$ B. Abbott, ${ }^{115}$ O. Abdinov, ${ }^{12, a}$ B. Abeloos, ${ }^{119}$ S. H. Abidi, ${ }^{161}$ O. S. AbouZeid, ${ }^{139}$ N. L. Abraham, ${ }^{151}$ H. Abramowicz, ${ }^{155}$ H. Abreu, ${ }^{154}$ R. Abreu, ${ }^{118}$ Y. Abulaiti, ${ }^{148 a, 148 b}$ B. S. Acharya, ${ }^{167 a, 167 b, b}$ S. Adachi, ${ }^{157}$ L. Adamczyk, ${ }^{41 a}$ J. Adelman, ${ }^{110}$ M. Adersberger, ${ }^{102}$ T. Adye, ${ }^{133}$ A. A. Affolder, ${ }^{139}$ Y. Afik, ${ }^{154}$ T. Agatonovic-Jovin, ${ }^{14}$ C. Agheorghiesei, ${ }^{28 c}$ J. A. Aguilar-Saavedra, ${ }^{128 a, 128 f}$ S. P. Ahlen, ${ }^{24}$ F. Ahmadov, ${ }^{68, c}$ G. Aielli, ${ }^{135 a, 135 b}$ S. Akatsuka, ${ }^{71}$ H. Akerstedt, ${ }^{148 a, 148 b}$ T. P. A. Åkesson, ${ }^{84}$ E. Akilli, ${ }^{52}$ A. V. Akimov, ${ }^{98}$ G. L. Alberghi, ${ }^{22 a, 22 b}$ J. Albert, ${ }^{172}$ P. Albicocco, ${ }^{50}$ M. J. Alconada Verzini ${ }^{74}$ S. C. Alderweireldt, ${ }^{108}$ M. Aleksa, ${ }^{32}$ I. N. Aleksandrov, ${ }^{68}$ C. Alexa, ${ }^{28 b}$ G. Alexander, ${ }^{155}$ T. Alexopoulos, ${ }^{10}$ M. Alhroob,${ }^{115}$ B. Ali, ${ }^{130}$ M. Aliev, ${ }^{76 a, 76 b}$ G. Alimonti, ${ }^{94 \mathrm{a}}$ J. Alison, ${ }^{33}$ S. P. Alkire, ${ }^{38}$ B. M. M. Allbrooke, ${ }^{151}$ B. W. Allen, ${ }^{118}$ P. P. Allport, ${ }^{19}$ A. Aloisio, ${ }^{106 a, 106 b}$ A. Alonso, ${ }^{39}$ F. Alonso,${ }^{74}$ C. Alpigiani, ${ }^{140}$ A. A. Alshehri, ${ }^{56}$ M. I. Alstaty,${ }^{88}$ B. Alvarez Gonzalez, ${ }^{32}$ D. Álvarez Piqueras, ${ }^{170}$ M. G. Alviggi, ${ }^{106 a, 106 b}$ B. T. Amadio, ${ }^{16}$ Y. Amaral Coutinho, ${ }^{26 a}$ C. Amelung, ${ }^{25}$ D. Amidei, ${ }^{92}$ S. P. Amor Dos Santos, ${ }^{128 a, 128 c}$ S. Amoroso, ${ }^{32}$ G. Amundsen, ${ }^{25}$ C. Anastopoulos,${ }^{141}$ L. S. Ancu,${ }^{52}$ N. Andari, ${ }^{19}$ T. Andeen, ${ }^{11}$ C. F. Anders, ${ }^{60 b}$ J. K. Anders, ${ }^{77}$ K. J. Anderson, ${ }^{33}$ A. Andreazza, ${ }^{94 a}, 94 \mathrm{~b}$ V. Andrei, ${ }^{60 a}$ S. Angelidakis, ${ }^{37}$ I. Angelozzi, ${ }^{109}$ A. Angerami, ${ }^{38}$ A. V. Anisenkov, ${ }^{111, \mathrm{~d}}$ N. Anjos, ${ }^{13}$ A. Annovi, ${ }^{126 a, 126 \mathrm{~b}}$ C. Antel,${ }^{60 \mathrm{a}}$ M. Antonelli, ${ }^{50}$ A. Antonov, ${ }^{100, a}$ D. J. Antrim, ${ }^{166}$ F. Anulli, ${ }^{134 a}$ M. Aoki, ${ }^{69}$ L. Aperio Bella, ${ }^{32}$ G. Arabidze, ${ }^{93}$ Y. Arai, ${ }^{69}$ J. P. Araque, ${ }^{128 a}$ V. Araujo Ferraz, ${ }^{26 a}$ A. T. H. Arce, ${ }^{48}$ R. E. Ardell, ${ }^{80}$ F. A. Arduh, ${ }^{74}$ J-F. Arguin, ${ }^{97}$ S. Argyropoulos,${ }^{66}$ M. Arik, ${ }^{20 a}$ A. J. Armbruster, ${ }^{32}$ L. J. Armitage, ${ }^{79}$ O. Arnaez,${ }^{161}$ H. Arnoldd ${ }^{51}$ M. Arratia, ${ }^{30}$ O. Arslan, ${ }^{23}$ A. Artamonov, ${ }^{99}$ G. Artoni, ${ }^{122}$ S. Artz, ${ }^{86}$ S. Asai, ${ }^{157}$ N. Asbah, ${ }^{45}$ A. Ashkenazi, ${ }^{155}$ L. Asquith, ${ }^{151}$ K. Assamagan, ${ }^{27}$ R. Astalos, ${ }^{146 a}$ M. Atkinson, ${ }^{169}$ N. B. Atlay, ${ }^{143}$ K. Augsten, ${ }^{130}$ G. Avolio, ${ }^{32}$ B. Axen, ${ }^{16}$ M. K. Ayoub, ${ }^{119}$ G. Azuelos, ${ }^{97, e}$ A. E. Baas,${ }^{60 a}$ M. J. Baca, ${ }^{19}$ H. Bachacou, ${ }^{138}$ K. Bachas, ${ }^{76 a, 76 b}$ M. Backes, ${ }^{122}$ P. Bagnaia, ${ }^{134 a, 134 b}$ M. Bahmani, ${ }^{42}$ H. Bahrasemani, ${ }^{144}$ J. T. Baines, ${ }^{133}$ M. Bajic,${ }^{39}$ O. K. Baker, ${ }^{179}$ E. M. Baldin, ${ }^{11, \mathrm{~d}}$ P. Balek, ${ }^{175}$ F. Balli, ${ }^{138}$ W. K. Balunas, ${ }^{124}$ E. Banas, ${ }^{42}$ A. Bandyopadhyay, ${ }^{23}$ Sw. Banerjee, ${ }^{176, \mathrm{f}}$ A. A. E. Bannoura, ${ }^{178}$ L. Barak, ${ }^{155}$ E. L. Barberio, ${ }^{91}$ D. Barberis, ${ }^{53 a, 53 b}$ M. Barbero, ${ }^{88}$ T. Barillari, ${ }^{103}$ M-S Barisits, ${ }^{32}$ J. T. Barkeloo, ${ }^{118}$ T. Barklow, ${ }^{145}$ N. Barlow, ${ }^{30}$ S. L. Barnes, ${ }^{36 c}$ B. M. Barnett, ${ }^{133}$ R. M. Barnett,,${ }^{16}$ Z. Barnovska-Blenessy, ${ }^{36 a}$ A. Baroncelli, ${ }^{136 a}$ G. Barone, ${ }^{25}$ A. J. Barr, ${ }^{122}$ L. Barranco Navarro, ${ }^{170}$ F. Barreiro, ${ }^{85}$ J. Barreiro Guimarães da Costa, ${ }^{35 a}$ R. Bartoldus, ${ }^{145}$ A. E. Barton, ${ }^{75}$ P. Bartos, ${ }^{146 a}$ A. Basalaev, ${ }^{125}$ A. Bassalat,${ }^{19, g}$ R. L. Bates, ${ }^{56}$ S. J. Batista ${ }^{161}$ J. R. Batley,${ }^{30}$ M. Battaglia, ${ }^{139}$ M. Bauce, ${ }^{134 a, 134 b}$ F. Bauer, ${ }^{138}$ H. S. Bawa, ${ }^{145, h}$ J. B. Beacham, ${ }^{113}$ M. D. Beattie, ${ }^{75}$ T. Beau, ${ }^{83}$ P. H. Beauchemin, ${ }^{165}$ P. Bechtle, ${ }^{23}$ H. P. Beck, ${ }^{18, i}$ H. C. Beck,${ }^{57}$ K. Becker, ${ }^{122}$ M. Becker, ${ }^{86}$ C. Becot, ${ }^{112}$ A. J. Beddall, ${ }^{20 \mathrm{~d}}$ A. Beddall, ${ }^{20 b}$ V. A. Bednyakov, ${ }^{68}$ M. Bedognetti, ${ }^{109}$ C. P. Bee, ${ }^{150}$ T. A. Beermann, ${ }^{32}$ M. Begalli, ${ }^{26 a}$ M. Begel, ${ }^{27}$ J. K. Behr, ${ }^{45}$ A. S. Bell, ${ }^{81}$ G. Bella, ${ }^{155}$ L. Bellagamba, ${ }^{22 a}$ A. Bellerive, ${ }^{31}$ M. Bellomo,${ }^{154}$ K. Belotskiy, ${ }^{100}$ O. Beltramello, ${ }^{32}$ N. L. Belyaev, ${ }^{100}$ O. Benary, ${ }^{155, a}$ D. Benchekroun, ${ }^{137 a}$ M. Bender, ${ }^{102}$ K. Bendtz, ${ }^{148 a, 148 b}$ N. Benekos, ${ }^{10}$ Y. Benhammou, ${ }^{155}$ E. Benhar Noccioli, ${ }^{179}$ J. Benitez, ${ }^{66}$ D. P. Benjamin, ${ }^{48}$ M. Benoit, ${ }^{52}$ J. R. Bensinger, ${ }^{25}$ S. Bentvelsen, ${ }^{109}$ L. Beresford, ${ }^{122}$ M. Beretta, ${ }^{50}$ D. Berge, ${ }^{109}$ E. Bergeaas Kuutmann, ${ }^{168}$ N. Berger, ${ }^{5}$ J. Beringer, ${ }^{16}$ S. Berlendis, ${ }^{58}$ N. R. Bernard, ${ }^{89}$ G. Bernardi, ${ }^{83}$ C. Bernius, ${ }^{145}$ F. U. Bernlochner, ${ }^{23}$ T. Berry, ${ }^{80}$ P. Berta, ${ }^{86}$ C. Bertella, ${ }^{35 a}$ G. Bertoli, ${ }^{148 a, 148 b}$

F. Bertolucci, ${ }^{126 a, 126 b}$ I. A. Bertram, ${ }^{75}$ C. Bertsche,${ }^{45}$ D. Bertsche, ${ }^{115}$ G. J. Besjes, ${ }^{39}$ O. Bessidskaia Bylund, ${ }^{148 a, 148 b}$ M. Bessner, ${ }^{45}$ N. Besson, ${ }^{138}$ C. Betancourt, ${ }^{51}$ A. Bethani, ${ }^{87}$ S. Bethke, ${ }^{103}$ A. J. Bevan, ${ }^{79}$ J. Beyer, ${ }^{103}$ R. M. Bianchi, ${ }^{127}$ O. Biebel,${ }^{102}$ D. Biedermann, ${ }^{17}$ R. Bielski, ${ }^{87}$ K. Bierwagen, ${ }^{86}$ N. V. Biesuz, ${ }^{126 a, 126 b}$ M. Biglietti, ${ }^{136 a}$ T. R. V. Billoud ${ }^{97}$ H. Bilokon, ${ }^{50}$ M. Bindi, ${ }^{57}$ A. Bingul, ${ }^{20 b}$ C. Bini, ${ }^{134 a, 134 b}$ S. Biondi, ${ }^{22 a, 22 b}$ T. Bisanz,${ }^{57}$ C. Bittrich, ${ }^{47}$ D. M. Bjergaard ${ }^{48}$ J. E. Black, ${ }^{145}$ K. M. Black, ${ }^{24}$ R. E. Blair, ${ }^{6}$ T. Blazek, ${ }^{146 a}$ I. Bloch, ${ }^{45}$ C. Blocker, ${ }^{25}$ A. Blue, ${ }^{56}$ W. Blum, ${ }^{86, a}$ U. Blumenschein, ${ }^{79}$ S. Blunier, ${ }^{34 a}$ G. J. Bobbink, ${ }^{109}$ V. S. Bobrovnikov, ${ }^{111, d}$ S. S. Bocchetta, ${ }^{84}$ A. Bocci, ${ }^{48}$ C. Bock, ${ }^{102}$ M. Boehler, ${ }^{51}$ D. Boerner, ${ }^{178}$ D. Bogavac, ${ }^{102}$ A. G. Bogdanchikov, ${ }^{111}$ C. Bohm, ${ }^{148 a}$ V. Boisvert, ${ }^{80}$ P. Bokan, ${ }^{168, j}$ T. Bold, ${ }^{41 a}$ A. S. Boldyrev, ${ }^{101}$ A. E. Bolz,${ }^{60 b}$ M. Bomben,${ }^{83}$ M. Bona,${ }^{79}$ M. Boonekamp,${ }^{138}$ A. Borisov, ${ }^{132}$ G. Borissov,${ }^{75}$ J. Bortfeldt,${ }^{32}$ D. Bortoletto, ${ }^{122}$ V. Bortolotto, ${ }^{62 a, 62 b, 62 \mathrm{c}}$ D. Boscherini, ${ }^{22 \mathrm{a}}$ M. Bosman, ${ }^{13} \mathrm{~J}$. D. Bossio Sola, ${ }^{29} \mathrm{~J}$. Boudreau, ${ }^{127} \mathrm{~J}$. Bouffard, ${ }^{2}$ 
E. V. Bouhova-Thacker, ${ }^{75}$ D. Boumediene,${ }^{37}$ C. Bourdarios,${ }^{119}$ S. K. Boutle ${ }^{56}$ A. Boveia, ${ }^{113}$ J. Boyd ${ }^{32}$ I. R. Boyko, ${ }^{68}$ J. Bracinik, ${ }^{19}$ A. Brandt, ${ }^{8}$ G. Brandt, ${ }^{57}$ O. Brandt, ${ }^{60 a}$ U. Bratzler, ${ }^{158}$ B. Brau, ${ }^{89}$ J. E. Brau, ${ }^{118}$ W. D. Breaden Madden, ${ }^{56}$

K. Brendlinger, ${ }^{45}$ A. J. Brennan, ${ }^{91}$ L. Brenner, ${ }^{109}$ R. Brenner, ${ }^{168}$ S. Bressler, ${ }^{175}$ D. L. Briglin, ${ }^{19}$ T. M. Bristow, ${ }^{49}$ D. Britton, ${ }^{56}$ D. Britzger, ${ }^{45}$ F. M. Brochu, ${ }^{30}$ I. Brock, ${ }^{23}$ R. Brock, ${ }^{93}$ G. Brooijmans, ${ }^{38}$ T. Brooks, ${ }^{80}$ W. K. Brooks, ${ }^{34 b}$ J. Brosamer, ${ }^{16}$

E. Brost, ${ }^{110}$ J. H Broughton, ${ }^{19}$ P. A. Bruckman de Renstrom, ${ }^{42}$ D. Bruncko ${ }^{146 b}$ A. Bruni ${ }^{22 a}$ G. Bruni, ${ }^{22 a}$ L. S. Bruni, ${ }^{109}$ BH Brunt, ${ }^{30}$ M. Bruschi, ${ }^{22 a}$ N. Bruscino, ${ }^{23}$ P. Bryant, ${ }^{33}$ L. Bryngemark, ${ }^{45}$ T. Buanes,${ }^{15}$ Q. Buat, ${ }^{144}$ P. Buchholz, ${ }^{143}$ A. G. Buckley, ${ }^{56}$ I. A. Budagov,${ }^{68}$ F. Buehrer ${ }^{51}$ M. K. Bugge, ${ }^{121}$ O. Bulekov, ${ }^{100}$ D. Bullock, ${ }^{8}$ T. J. Burch, ${ }^{110}$ S. Burdin, ${ }^{77}$ C. D. Burgard, ${ }^{51}$ A. M. Burger, ${ }^{5}$ B. Burghgrave, ${ }^{110}$ K. Burka, ${ }^{42}$ S. Burke, ${ }^{133}$ I. Burmeister, ${ }^{46}$ J. T. P. Burr, ${ }^{122}$ E. Busato, ${ }^{37}$ D. Büscher, ${ }^{51}$ V. Büscher, ${ }^{86}$ P. Bussey, ${ }^{56}$ J. M. Butler, ${ }^{24}$ C. M. Buttar,${ }^{56}$ J. M. Butterworth ${ }^{81}$ P. Butti, ${ }^{32}$ W. Buttinger ${ }^{27}$ A. Buzatu, ${ }^{35 \mathrm{c}}$ A. R. Buzykaev, ${ }^{11, \mathrm{~d}}$ S. Cabrera Urbán, ${ }^{170}$ D. Caforio, ${ }^{130}$ V. M. Cairo, ${ }^{40,40 \mathrm{~b}}$ O. Cakir, ${ }^{4 \mathrm{a}}$ N. Calace, ${ }^{52}$ P. Calafiura, ${ }^{16}$ A. Calandri, ${ }^{88}$ G. Calderini,${ }^{83}$ P. Calfayan, ${ }^{64}$ G. Callea,${ }^{40 a}{ }^{40 b}$ L. P. Caloba, ${ }^{26 a}$ S. Calvente Lopez, ${ }^{85}$ D. Calvet,${ }^{37}$ S. Calvet,${ }^{37}$ T. P. Calvet ${ }^{88}$ R. Camacho Toro, ${ }^{33}$ S. Camarda ${ }^{32}$ P. Camarri, ${ }^{135 a, 135 b}$ D. Cameron, ${ }^{121}$ R. Caminal Armadans, ${ }^{169}$ C. Camincher ${ }^{58}$ S. Campana, ${ }^{32}$ M. Campanelli, ${ }^{81}$ A. Camplani, ${ }^{94 a, 94 b}$ A. Campoverde, ${ }^{143}$ V. Canale,${ }^{106 a, 106 b}$ M. Cano Bret, ${ }^{36 c}$ J. Cantero, ${ }^{116}$ T. Cao, ${ }^{155}$ M. D. M. Capeans Garrido, ${ }^{32}$ I. Caprini, ${ }^{28 b}$ M. Caprini, ${ }^{28 b}$ M. Capua, ${ }^{40 a, 40 b}$ R. M. Carbone, ${ }^{38}$ R. Cardarelli, ${ }^{135 a}$ F. Cardillo, ${ }^{51}$ I. Carli, ${ }^{131}$ T. Carli, ${ }^{32}$ G. Carlino, ${ }^{106 a}$ B. T. Carlson, ${ }^{127}$ L. Carminati, ${ }^{94 a, 94 b}$
R. M. D. Carney, ${ }^{148 a, 148 b}$ S. Caron, ${ }^{108}$ E. Carquin, ${ }^{34 b}$ S. Carrá, ${ }^{94 a, 94 b}$ G. D. Carrillo-Montoya, ${ }^{32}$ D. Casadei, ${ }^{19}$

M. P. Casado, ${ }^{13, k}$ M. Casolino, ${ }^{13}$ D. W. Casper, ${ }^{166}$ R. Castelijn, ${ }^{109}$ V. Castillo Gimenez, ${ }^{170}$ N. F. Castro, ${ }^{128 a, 1}$ A. Catinaccio, ${ }^{32}$

J. R. Catmore, ${ }^{121}$ A. Cattai, ${ }^{32}$ J. Caudron, ${ }^{23}$ V. Cavaliere, ${ }^{169}$ E. Cavallaro,${ }^{13}$ D. Cavalli, ${ }^{94 a}$ M. Cavalli-Sforza ${ }^{13}$

V. Cavasinni, ${ }^{126 a, 126 b}$ E. Celebi, ${ }^{20 a}$ F. Ceradini, ${ }^{136 a, 136 b}$ L. Cerda Alberich, ${ }^{170}$ A. S. Cerqueira, ${ }^{26 b}$ A. Cerri, ${ }^{151}$

L. Cerrito, ${ }^{135 a, 135 b}$ F. Cerutti, ${ }^{16}$ A. Cervelli, ${ }^{18}$ S. A. Cetin, ${ }^{20 c}$ A. Chafaq, ${ }^{137 a}$ D. Chakraborty, ${ }^{110}$ S. K. Chan, ${ }^{59}$ W. S. Chan, ${ }^{109}$ Y. L. Chan, ${ }^{62 \mathrm{a}}$ P. Chang, ${ }^{169}$ J. D. Chapman, ${ }^{30}$ D. G. Charlton, ${ }^{19}$ C. C. Chau, ${ }^{31}$ C. A. Chavez Barajas, ${ }^{151}$ S. Che, ${ }^{113}$

S. Cheatham, ${ }^{167 a, 167 \mathrm{c}}$ A. Chegwidden, ${ }^{93}$ S. Chekanov, ${ }^{6}$ S. V. Chekulaev, ${ }^{163 a}$ G. A. Chelkov, ${ }^{68, m}$ M. A. Chelstowska, ${ }^{32}$ C. Chen, ${ }^{67}$ H. Chen, ${ }^{27}$ J. Chen, ${ }^{36 a}$ S. Chen, ${ }^{35 b}$ S. Chen, ${ }^{157}$ X. Chen, ${ }^{35 c, n}$ Y. Chen, ${ }^{70}$ H. C. Cheng, ${ }^{92}$ H. J. Cheng, ${ }^{35 a}$

A. Cheplakov, ${ }^{68}$ E. Cheremushkina, ${ }^{132}$ R. Cherkaoui El Moursli, ${ }^{137 e}$ E. Cheu, ${ }^{7}$ K. Cheung, ${ }^{63}$ L. Chevalier,${ }^{138}$ V. Chiarella, ${ }^{50}$ G. Chiarelli, ${ }^{126 a, 126 b}$ G. Chiodini, ${ }^{76 a}$ A. S. Chisholm, ${ }^{32}$ A. Chitan, ${ }^{28 b}$ Y. H. Chiu, ${ }^{172}$ M. V. Chizhov, ${ }^{68}$ K. Choi, ${ }^{64}$ A. R. Chomont, ${ }^{37}$ S. Chouridou, ${ }^{156}$ Y. S. Chow, ${ }^{62 a}$ V. Christodoulou, ${ }^{81}$ M. C. Chu, ${ }^{62 a}$ J. Chudoba, ${ }^{129}$ A. J. Chuinard, ${ }^{90}$ J. J. Chwastowski, ${ }^{42}$ L. Chytka ${ }^{117}$ A. K. Ciftci, ${ }^{4 a}$ D. Cinca, ${ }^{46}$ V. Cindro, ${ }^{78}$ I. A. Cioara, ${ }^{23}$ C. Ciocca, ${ }^{22 a, 22 b}$ A. Ciocio, ${ }^{16}$ F. Cirotto, ${ }^{106 a, 106 b}$ Z. H. Citron, ${ }^{175}$ M. Citterio, ${ }^{94 a}$ M. Ciubancan, ${ }^{28 b}$ A. Clark,${ }^{52}$ B. L. Clark,${ }^{59}$ M. R. Clark, ${ }^{38}$ P. J. Clark,${ }^{49}$ R. N. Clarke, ${ }^{16}$ C. Clement, ${ }^{148 a, 148 b}$ Y. Coadou, ${ }^{88}$ M. Cobal, ${ }^{167 a, 167 \mathrm{c}}$ A. Coccaro,${ }^{52}$ J. Cochran, ${ }^{67}$ L. Colasurdo, ${ }^{108}$ B. Cole, ${ }^{38}$ A. P. Colijn, ${ }^{109}$ J. Collot, ${ }^{58}$ T. Colombo, ${ }^{166}$ P. Conde Muiño, ${ }^{128 a, 128 b}$ E. Coniavitis, ${ }^{51}$ S. H. Connell, ${ }^{147 b}$ I. A. Connelly, ${ }^{87}$ S. Constantinescu, ${ }^{28 b}$ G. Conti, ${ }^{32}$ F. Conventi, ${ }^{106 a, 0}$ M. Cooke, ${ }^{16}$ A. M. Cooper-Sarkar, ${ }^{122}$ F. Cormier, ${ }^{171}$ K. J. R. Cormier, ${ }^{161}$ M. Corradi, ${ }^{134 a, 134 b}$ F. Corriveau, ${ }^{90, p}$ A. Cortes-Gonzalez, ${ }^{32}$ G. Cortiana, ${ }^{103}$ G. Costa, ${ }^{94 a}$ M. J. Costa, ${ }^{170}$ D. Costanzo, ${ }^{141}$ G. Cottin, ${ }^{30}$ G. Cowan, ${ }^{80}$ B. E. Cox,${ }^{87}$ K. Cranmer, ${ }^{112}$ S. J. Crawley, ${ }^{56}$ R. A. Creager, ${ }^{124}$ G. Cree, ${ }^{31}$ S. Crépé-Renaudin, ${ }^{58}$ F. Crescioli ${ }^{83}$ W. A. Cribbs, ${ }^{148 a, 148 b}$ M. Cristinziani, ${ }^{23}$ V. Croft, ${ }^{108}$ G. Crosetti, ${ }^{40 a, 40 b}$ A. Cueto, ${ }^{85}$

T. Cuhadar Donszelmann, ${ }^{141}$ A. R. Cukierman, ${ }^{145}$ J. Cummings, ${ }^{179}$ M. Curatolo,${ }^{50}$ J. Cúth, ${ }^{86}$ S. Czekierda, ${ }^{42}$ P. Czodrowski, ${ }^{32}$ G. D'amen, ${ }^{22 a, 22 b}$ S. D'Auria, ${ }^{56}$ L. D'eramo, ${ }^{83}$ M. D’Onofrio, ${ }^{77}$ M. J. Da Cunha Sargedas De Sousa, ${ }^{128 a, 128 b}$ C. Da Via ${ }^{87}$ W. Dabrowski, ${ }^{41 a}$ T. Dado, ${ }^{146 a}$ T. Dai,${ }^{92}$ O. Dale, ${ }^{15}$ F. Dallaire, ${ }^{97}$ C. Dallapiccola, ${ }^{89}$ M. Dam,${ }^{39}$ J. R. Dandoy, ${ }^{124}$ M. F. Daneri, ${ }^{29}$ N. P. Dang, ${ }^{176}$ A. C. Daniells, ${ }^{19}$ N. S. Dann, ${ }^{87}$ M. Danninger, ${ }^{171}$ M. Dano Hoffmann, ${ }^{138}$ V. Dao, ${ }^{150}$ G. Darbo, ${ }^{53 a}$ S. Darmora, ${ }^{8}$ J. Dassoulas, ${ }^{3}$ A. Dattagupta, ${ }^{118}$ T. Daubney, ${ }^{45}$ W. Davey, ${ }^{23}$ C. David ${ }^{45}$ T. Davidek,${ }^{131}$ D. R. Davis,${ }^{48}$ P. Davison, ${ }^{81}$ E. Dawe,${ }^{91}$ I. Dawson, ${ }^{141}$ K. De,${ }^{8}$ R. de Asmundis, ${ }^{106 a}$ A. De Benedetti, ${ }^{115}$ S. De Castro, ${ }^{22 a, 22 b}$ S. De Cecco, ${ }^{83}$ N. De Groot, ${ }^{108}$ P. de Jong, ${ }^{109}$ H. De la Torre, ${ }^{93}$ F. De Lorenzi, ${ }^{67}$ A. De Maria, ${ }^{57}$ D. De Pedis, ${ }^{134 a}$ A. De Salvo, ${ }^{134 a}$ U. De Sanctis, ${ }^{135 a, 135 b}$ A. De Santo,${ }^{151}$ K. De Vasconcelos Corga,${ }^{88}$ J. B. De Vivie De Regie, ${ }^{119}$ R. Debbe, ${ }^{27}$ C. Debenedetti, ${ }^{139}$ D. V. Dedovich ${ }^{68}$ N. Dehghanian, ${ }^{3}$ I. Deigaard, ${ }^{109}$ M. Del Gaudio,${ }^{40 a, 40 b}$ J. Del Peso,${ }^{85}$ D. Delgove, ${ }^{119}$ F. Deliot, ${ }^{138}$ C. M. Delitzsch, ${ }^{7}$ A. Dell'Acqua ${ }^{32}$ L. Dell'Asta, ${ }^{24}$ M. Dell'Orso, ${ }^{126 a, 126 b}$ M. Della Pietra, ${ }^{106 a, 106 b}$ D. della Volpe,${ }^{52}$ M. Delmastro, ${ }^{5}$ C. Delporte, ${ }^{119}$ P. A. Delsart, ${ }^{58}$ D. A. DeMarco, ${ }^{161}$ S. Demers,${ }^{179}$ M. Demichev, ${ }^{68}$ A. Demilly, ${ }^{83}$ S. P. Denisov, ${ }^{132}$ D. Denysiuk, ${ }^{138}$ D. Derendarz,${ }^{42}$ J. E. Derkaoui, ${ }^{137 d}$ F. Derue,${ }^{83}$ P. Dervan,${ }^{77}$ K. Desch, ${ }^{23}$ C. Deterre ${ }^{45}$ K. Dette, ${ }^{161}$ M. R. Devesa, ${ }^{29}$ P. O. Deviveiros, ${ }^{32}$ A. Dewhurst,${ }^{133}$ S. Dhaliwal, ${ }^{25}$ F. A. Di Bello, ${ }^{52}$ A. Di Ciaccio, ${ }^{135 a, 135 b}$ L. Di Ciaccio, ${ }^{5}$ W. K. Di Clemente, ${ }^{124}$ C. Di Donato, ${ }^{106 a, 106 b}$ A. Di Girolamo, ${ }^{32}$ B. Di Girolamo, ${ }^{32}$ B. Di Micco, ${ }^{136 a, 136 b}$ R. Di Nardo, ${ }^{32}$ K. F. Di Petrillo, ${ }^{59}$ A. Di Simone, ${ }^{51}$ R. Di Sipio, ${ }^{161}$ D. Di Valentino, ${ }^{31}$ C. Diaconu, ${ }^{88}$ 
M. Diamond ${ }^{161}$ F. A. Dias, ${ }^{39}$ M. A. Diaz,${ }^{34 a}$ E. B. Diehl,${ }^{92}$ J. Dietrich, ${ }^{17}$ S. Díez Cornell, ${ }^{45}$ A. Dimitrievska, ${ }^{14}$ J. Dingfelder, ${ }^{23}$ P. Dita, ${ }^{28 b}$ S. Dita, ${ }^{28 b}$ F. Dittus, ${ }^{32}$ F. Djama ${ }^{88}$ T. Djobava, ${ }^{5 b}$ J. I. Djuvsland, ${ }^{60 a}$ M. A. B. do Vale,${ }^{26 c}$ D. Dobos, ${ }^{32}$ M. Dobre, ${ }^{28 b}$ C. Doglioni, ${ }^{84}$ J. Dolejsi, ${ }^{131}$ Z. Dolezal, ${ }^{131}$ M. Donadelli, ${ }^{26 \mathrm{~d}}$ S. Donati, ${ }^{126 a, 126 b}$ P. Dondero, ${ }^{123 a, 123 b}$ J. Donini, ${ }^{37}$ J. Dopke, ${ }^{133}$ A. Doria, ${ }^{106 a}$ M. T. Dova,${ }^{74}$ A. T. Doyle,${ }^{56}$ E. Drechsler, ${ }^{57}$ M. Dris,${ }^{10}$ Y. Du, ${ }^{36 b}$ J. Duarte-Campderros, ${ }^{155}$ A. Dubreuil,${ }^{52}$ E. Duchovni, ${ }^{175}$ G. Duckeck, ${ }^{102}$ A. Ducourthial, ${ }^{83}$ O. A. Ducu,,${ }^{97, q}$ D. Duda, ${ }^{109}$ A. Dudarev, ${ }^{32}$ A. Chr. Dudder, ${ }^{86}$ E. M. Duffield, ${ }^{16}$ L. Duflot, ${ }^{119}$ M. Dührssen, ${ }^{32}$ M. Dumancic, ${ }^{175}$ A. E. Dumitriu, ${ }^{28 b}$ A. K. Duncan, ${ }^{56}$ M. Dunford, ${ }^{60 a}$ H. Duran Yildiz,${ }^{4 a}$ M. Düren, ${ }^{55}$ A. Durglishvili, ${ }^{54 b}$ D. Duschinger,${ }^{47}$ B. Dutta, ${ }^{45}$ M. Dyndal, ${ }^{45}$ B. S. Dziedzic, ${ }^{42}$ C. Eckardt, ${ }^{45}$ K. M. Ecker, ${ }^{103}$ R. C. Edgar,${ }^{92}$ T. Eifert, ${ }^{32}$ G. Eigen, ${ }^{15}$ K. Einsweiler, ${ }^{16}$ T. Ekelof, ${ }^{168}$ M. El Kacimi, ${ }^{137 \mathrm{c}}$ R. El Kosseifi, ${ }^{88}$ V. Ellajosyula, ${ }^{88}$ M. Ellert, ${ }^{168}$ S. Elles, ${ }^{5}$ F. Ellinghaus, ${ }^{178}$ A. A. Elliot, ${ }^{172}$ N. Ellis, ${ }^{32}$ J. Elmsheuser, ${ }^{27}$ M. Elsing, ${ }^{32}$ D. Emeliyanov, ${ }^{133}$ Y. Enari, ${ }^{157}$ O. C. Endner, ${ }^{86}$ J. S. Ennis, ${ }^{173}$ J. Erdmann, ${ }^{46}$ A. Ereditato, ${ }^{18}$ M. Ernst, ${ }^{27}$ S. Errede, ${ }^{169}$ M. Escalier,${ }^{119}$ C. Escobar, ${ }^{170}$ B. Esposito,${ }^{50}$ O. Estrada Pastor,${ }^{170}$ A. I. Etienvre, ${ }^{138}$

E. Etzion, ${ }^{155}$ H. Evans, ${ }^{64}$ A. Ezhilov, ${ }^{125}$ M. Ezzi,${ }^{137 \mathrm{e}}$ F. Fabbri, ${ }^{22 a, 22 b}$ L. Fabbri, ${ }^{22 a, 22 b}$ V. Fabiani, ${ }^{108}$ G. Facini,${ }^{81}$ R. M. Fakhrutdinov, ${ }^{132}$ S. Falciano, ${ }^{134 a}$ R. J. Falla, ${ }^{81}$ J. Faltova, ${ }^{32}$ Y. Fang, ${ }^{35 a}$ M. Fanti $,{ }^{94 a}, 94 b$ A. Farbin, ${ }^{8}$ A. Farilla, ${ }^{136 a}$ C. Farina, ${ }^{127}$ E. M. Farina, ${ }^{123 a, 123 b}$ T. Farooque,${ }^{93}$ S. Farrell, ${ }^{16}$ S. M. Farrington, ${ }^{173}$ P. Farthouat, ${ }^{32}$ F. Fassi,,${ }^{137}$ P. Fassnacht,${ }^{32}$ D. Fassouliotis, ${ }^{9}$ M. Faucci Giannelli, ${ }^{80}$ A. Favareto, ${ }^{53 \mathrm{a}, 53 \mathrm{~b}}$ W. J. Fawcett, ${ }^{122}$ L. Fayard, ${ }^{119}$ O. L. Fedin, ${ }^{125, \mathrm{r}}$ W. Fedorko, ${ }^{171}$ S. Feigl ${ }^{121}$ L. Feligioni ${ }^{88}$ C. Feng, ${ }^{36 b}$ E. J. Feng, ${ }^{32}$ H. Feng, ${ }^{92}$ M. J. Fenton, ${ }^{56}$ A. B. Fenyuk ${ }^{132}$ L. Feremenga, ${ }^{8}$

P. Fernandez Martinez, ${ }^{170}$ S. Fernandez Perez,${ }^{13}$ J. Ferrando, ${ }^{45}$ A. Ferrari, ${ }^{168}$ P. Ferrari, ${ }^{109}$ R. Ferrari, ${ }^{123 a}$ D. E. Ferreira de Lima, ${ }^{60 b}$ A. Ferrer, ${ }^{170}$ D. Ferrere,${ }^{52}$ C. Ferretti ${ }^{92}$ F. Fiedler, ${ }^{86}$ A. Filipčič ${ }^{78}$ M. Filipuzzi,${ }^{45}$ F. Filthaut,${ }^{108}$ M. Fincke-Keeler, ${ }^{172}$ K. D. Finelli, ${ }^{152}$ M. C. N. Fiolhais, ${ }^{128 a, 128 c, s}$ L. Fiorini, ${ }^{170}$ A. Fischer, ${ }^{2}$ C. Fischer, ${ }^{13}$ J. Fischer, ${ }^{178}$ W. C. Fisher, ${ }^{93}$ N. Flaschel, ${ }^{45}$ I. Fleck,${ }^{143}$ P. Fleischmann, ${ }^{92}$ R. R. M. Fletcher, ${ }^{124}$ T. Flick, ${ }^{178}$ B. M. Flierl,,${ }^{102}$ L. R. Flores Castillo, ${ }^{62 a}$ M. J. Flowerdew, ${ }^{103}$ G. T. Forcolin, ${ }^{87}$ A. Formica, ${ }^{138}$ F. A. Förster, ${ }^{13}$ A. Forti, ${ }^{87}$ A. G. Foster, ${ }^{19}$ D. Fournier, ${ }^{119}$ H. Fox,${ }^{75}$ S. Fracchia, ${ }^{141}$ P. Francavilla ${ }^{83}$ M. Franchini, ${ }^{22 a, 22 b}$ S. Franchino, ${ }^{60 a}$ D. Francis,${ }^{32}$ L. Franconi, ${ }^{121}$ M. Franklin ${ }^{59}$ M. Frate, ${ }^{166}$ M. Fraternali, ${ }^{123 a, 123 b}$ D. Freeborn, ${ }^{81}$ S. M. Fressard-Batraneanu,${ }^{32}$ B. Freund,${ }^{97}$ D. Froidevaux,${ }^{32}$ J. A. Frost, ${ }^{122}$ C. Fukunaga, ${ }^{158}$ T. Fusayasu, ${ }^{104}$ J. Fuster, ${ }^{170}$ C. Gabaldon, ${ }^{58}$ O. Gabizon, ${ }^{154}$ A. Gabrielli, ${ }^{22 a, 22 b}$ A. Gabrielli, ${ }^{16}$ G. P. Gach, ${ }^{41 a}$ S. Gadatsch, ${ }^{32}$ S. Gadomski, ${ }^{80}$ G. Gagliardi, ${ }^{53 a, 53 b}$ L. G. Gagnon, ${ }^{97}$ C. Galea, ${ }^{108}$ B. Galhardo, ${ }^{128 a}, 128 c$

E. J. Gallas, ${ }^{122}$ B. J. Gallop, ${ }^{133}$ P. Gallus, ${ }^{130}$ G. Galster, ${ }^{39}$ K. K. Gan, ${ }^{113}$ S. Ganguly, ${ }^{37}$ Y. Gao, ${ }^{77}$ Y. S. Gao, ${ }^{145, h}$

F. M. Garay Walls, ${ }^{49}$ C. García, ${ }^{170}$ J. E. García Navarro, ${ }^{170}$ J. A. García Pascual, ${ }^{35 a}$ M. Garcia-Sciveres, ${ }^{16}$ R. W. Gardner, ${ }^{33}$

N. Garelli, ${ }^{145}$ V. Garonne, ${ }^{121}$ A. Gascon Bravo,${ }^{45}$ K. Gasnikova, ${ }^{45}$ C. Gatti, ${ }^{50}$ A. Gaudiello, ${ }^{53 a, 53 b}$ G. Gaudio, ${ }^{123 a}$ I. L. Gavrilenko, ${ }^{98}$ C. Gay, ${ }^{171}$ G. Gaycken, ${ }^{23}$ E. N. Gazis, ${ }^{10}$ C. N. P. Gee, ${ }^{133}$ J. Geisen, ${ }^{57}$ M. Geisen, ${ }^{86}$ M. P. Geisler, ${ }^{60 a}$ K. Gellerstedt, ${ }^{148 a, 148 b}$ C. Gemme,${ }^{53 \mathrm{a}}$ M. H. Genest,${ }^{58}$ C. Geng, ${ }^{92}$ S. Gentile,${ }^{134 a, 134 b}$ C. Gentsos,${ }^{156}$ S. George ${ }^{80}$ D. Gerbaudo, ${ }^{13}$ A. Gershon, ${ }^{155}$ G. Geßner, ${ }^{46}$ S. Ghasemi, ${ }^{143}$ M. Ghneimat, ${ }^{23}$ B. Giacobbe, ${ }^{22 a}$ S. Giagu, ${ }^{134 a, 134 b}$ N. Giangiacomi, ${ }^{22 a, 22 b}$ P. Giannetti, ${ }^{126 a, 126 b}$ S. M. Gibson, ${ }^{80}$ M. Gignac, ${ }^{171}$ M. Gilchriese, ${ }^{16}$ D. Gillberg, ${ }^{31}$ G. Gilles,${ }^{178}$ D. M. Gingrich, ${ }^{3, \mathrm{e}}$ M. P. Giordani, ${ }^{167 \mathrm{a}, 167 \mathrm{c}}$ F. M. Giorgi, ${ }^{22 \mathrm{a}}$ P. F. Giraud,${ }^{138}$ P. Giromini, ${ }^{59}$ G. Giugliarelli, ${ }^{167 \mathrm{a}, 167 \mathrm{c}}$ D. Giugni, ${ }^{94 \mathrm{a}}$

F. Giuli, ${ }^{122}$ C. Giuliani, ${ }^{103}$ M. Giulini, ${ }^{60 b}$ B. K. Gjelsten, ${ }^{121}$ S. Gkaitatzis, ${ }^{156}$ I. Gkialas, ${ }^{9, t}$ E. L. Gkougkousis, ${ }^{13}$ P. Gkountoumis, ${ }^{10}$ L. K. Gladilin, ${ }^{101}$ C. Glasman, ${ }^{85}$ J. Glatzer, ${ }^{13}$ P. C. F. Glaysher, ${ }^{45}$ A. Glazov ${ }^{45}$ M. Goblirsch-Kolb, ${ }^{25}$ J. Godlewski, ${ }^{42}$ S. Goldfarb, ${ }^{91}$ T. Golling, ${ }^{52}$ D. Golubkov, ${ }^{132}$ A. Gomes, ${ }^{128 a, 128 b, 128 d}$ R. Gonçalo, ${ }^{128 a}$ R. Goncalves Gama, ${ }^{26 a}$ J. Goncalves Pinto Firmino Da Costa, ${ }^{138}$ G. Gonella, ${ }^{51}$ L. Gonella, ${ }^{19}$ A. Gongadze, ${ }^{68}$ S. González de la Hoz, ${ }^{170}$ S. Gonzalez-Sevilla, ${ }^{52}$ L. Goossens, ${ }^{32}$ P. A. Gorbounov, ${ }^{99}$ H. A. Gordon, ${ }^{27}$ I. Gorelov, ${ }^{107}$ B. Gorini, ${ }^{32}$ E. Gorini, ${ }^{76 a, 76 b}$ A. Gorišek, ${ }^{78}$ A. T. Goshaw, ${ }^{48}$ C. Gössling, ${ }^{46}$ M. I. Gostkin, ${ }^{68}$ C. A. Gottardo, ${ }^{23}$ C. R. Goudet, ${ }^{119}$ D. Goujdami, ${ }^{137 c}$ A. G. Goussiou, ${ }^{140}$ N. Govender, ${ }^{147 b, u}$ E. Gozani, ${ }^{154}$ L. Graber, ${ }^{57}$ I. Grabowska-Bold, ${ }^{41 a}$ P. O. J. Gradin, ${ }^{168}$ J. Gramling, ${ }^{166}$ E. Gramstad, ${ }^{121}$ S. Grancagnolo, ${ }^{17}$ V. Gratchev, ${ }^{125}$ P. M. Gravila, ${ }^{28 f}$ C. Gray, ${ }^{56}$ H. M. Gray, ${ }^{16}$ Z. D. Greenwood, ${ }^{82, v}$ C. Grefe, ${ }^{23}$ K. Gregersen, ${ }^{81}$ I. M. Gregor, ${ }^{45}$ P. Grenier, ${ }^{145}$ K. Grevtsov, ${ }^{5}$ J. Griffiths, ${ }^{8}$ A. A. Grillo, ${ }^{139}$ K. Grimm, ${ }^{75}$ S. Grinstein, ${ }^{13, w}$ Ph. Gris, ${ }^{37}$ J.-F. Grivaz, ${ }^{119}$ S. Groh ${ }^{86}$ E. Gross, ${ }^{175}$ J. Grosse-Knetter,${ }^{57}$ G. C. Grossi,${ }^{82}$ Z. J. Grout ${ }^{81}$ A. Grummer, ${ }^{107}$ L. Guan, ${ }^{92}$ W. Guan, ${ }^{176}$ J. Guenther, ${ }^{65}$ F. Guescini, ${ }^{163 a}$ D. Guest, ${ }^{166}$ O. Gueta, ${ }^{155}$ B. Gui, ${ }^{113}$ E. Guido, ${ }^{53 a, 53 b}$ T. Guillemin, ${ }^{5}$ S. Guindon, ${ }^{2}$ U. Gul, ${ }^{56}$ C. Gumpert, ${ }^{32}$ J. Guo, ${ }^{36 \mathrm{c}}$ W. Guo, ${ }^{92}$ Y. Guo, ${ }^{36 a}$ R. Gupta, ${ }^{43}$ S. Gupta, ${ }^{122}$ G. Gustavino, ${ }^{115}$ B. J. Gutelman, ${ }^{154}$ P. Gutierrez, ${ }^{115}$ N. G. Gutierrez Ortiz,${ }^{81}$ C. Gutschow, ${ }^{81}$ C. Guyot,${ }^{138}$ M. P. Guzik, ${ }^{41 a}$ C. Gwenlan, ${ }^{122}$ C. B. Gwilliam, ${ }^{77}$ A. Haas, ${ }^{112}$ C. Haber, ${ }^{16}$ H. K. Hadavand, ${ }^{8}$ N. Haddad, ${ }^{137 e}$ A. Hadef, ${ }^{88}$ S. Hageböck, ${ }^{23}$ M. Hagihara, ${ }^{164}$ H. Hakobyan, ${ }^{180, a}$ M. Haleem, ${ }^{45}$ J. Haley,${ }^{116}$ G. Halladjian, ${ }^{93}$ G. D. Hallewell,${ }^{88}$ K. Hamacher, ${ }^{178}$ P. Hamal, ${ }^{117}$ K. Hamano, ${ }^{172}$ A. Hamilton, ${ }^{147 a}$ G. N. Hamity, ${ }^{141}$ P. G. Hamnett, ${ }^{45}$ L. Han, ${ }^{36 a}$ S. Han, ${ }^{35 a}$ K. Hanagaki, ${ }^{69, x}$ 
K. Hanawa, ${ }^{157}$ M. Hance, ${ }^{139}$ B. Haney, ${ }^{124}$ P. Hanke, ${ }^{60 a}$ J. B. Hansen, ${ }^{39}$ J. D. Hansen, ${ }^{39}$ M. C. Hansen,${ }^{23}$ P. H. Hansen, ${ }^{39}$ K. Hara ${ }^{164}$ A. S. Hard, ${ }^{176}$ T. Harenberg, ${ }^{178}$ F. Hariri, ${ }^{119}$ S. Harkusha, ${ }^{95}$ R. D. Harrington, ${ }^{49}$ P. F. Harrison, ${ }^{173}$

N. M. Hartmann, ${ }^{102}$ Y. Hasegawa, ${ }^{142}$ A. Hasib ${ }^{49}$ S. Hassani, ${ }^{138}$ S. Haug,${ }^{18}$ R. Hauser,${ }^{93}$ L. Hauswald, ${ }^{47}$ L. B. Havener, ${ }^{38}$ M. Havranek, ${ }^{130}$ C. M. Hawkes, ${ }^{19}$ R. J. Hawkings, ${ }^{32}$ D. Hayakawa, ${ }^{159}$ D. Hayden, ${ }^{93}$ C. P. Hays, ${ }^{122}$ J. M. Hays,${ }^{79}$ H. S. Hayward ${ }^{77}$ S. J. Haywood, ${ }^{133}$ S. J. Head, ${ }^{19}$ T. Heck ${ }^{86}$ V. Hedberg, ${ }^{84}$ L. Heelan, ${ }^{8}$ S. Heer, ${ }^{23}$ K. K. Heidegger ${ }^{51}$ S. Heim, ${ }^{45}$ T. Heim, ${ }^{16}$ B. Heinemann, ${ }^{45, y}$ J. J. Heinrich, ${ }^{102}$ L. Heinrich,${ }^{12}$ C. Heinz, ${ }^{55}$ J. Hejbal, ${ }^{129}$ L. Helary, ${ }^{32}$ A. Held, ${ }^{171}$ S. Hellman, ${ }^{148 a, 148 b}$ C. Helsens, ${ }^{32}$ R. C. W. Henderson, ${ }^{75}$ Y. Heng,,${ }^{176}$ S. Henkelmann, ${ }^{171}$ A. M. Henriques Correia, ${ }^{32}$ S. Henrot-Versille, ${ }^{119}$ G. H. Herbert, ${ }^{17}$ H. Herde, ${ }^{25}$ V. Herget, ${ }^{177}$ Y. Hernández Jiménez, ${ }^{147 c}$ H. Herr, ${ }^{86}$ G. Herten, ${ }^{51}$ R. Hertenberger, ${ }^{102}$ L. Hervas, ${ }^{32}$ T. C. Herwig, ${ }^{124}$ G. G. Hesketh, ${ }^{81}$ N. P. Hessey, ${ }^{163 a}$ J. W. Hetherly, ${ }^{43}$ S. Higashino, ${ }^{69}$ E. Higón-Rodriguez, ${ }^{170}$ K. Hildebrand, ${ }^{33}$ E. Hill, ${ }^{172}$ J. C. Hill, ${ }^{30}$ K. H. Hiller, ${ }^{45}$ S. J. Hillier, ${ }^{19}$ M. Hils, ${ }^{47}$ I. Hinchliffe, ${ }^{16}$ M. Hirose,${ }^{51}$ D. Hirschbuehl, ${ }^{178}$ B. Hiti, ${ }^{78}$ O. Hladik, ${ }^{129}$ X. Hoad,${ }^{49}$ J. Hobbs, ${ }^{150}$ N. Hod, ${ }^{163 a}$ M. C. Hodgkinson, ${ }^{141}$ P. Hodgson, ${ }^{141}$ A. Hoecker, ${ }^{32}$ M. R. Hoeferkamp, ${ }^{107}$ F. Hoenig, ${ }^{102}$ D. Hohn, ${ }^{23}$ T. R. Holmes,${ }^{33}$ M. Homann, ${ }^{46}$ S. Honda, ${ }^{164}$ T. Honda, ${ }^{69}$ T. M. Hong, ${ }^{127}$ B. H. Hooberman, ${ }^{169}$ W. H. Hopkins, ${ }^{118}$ Y. Horii, ${ }^{105}$ A. J. Horton, ${ }^{144}$ J-Y. Hostachy, ${ }^{58}$ S. Hou, ${ }^{153}$ A. Hoummada, ${ }^{137 a}$ J. Howarth,${ }^{87}$ J. Hoya, ${ }^{74}$ M. Hrabovsky, ${ }^{117}$ J. Hrdinka, ${ }^{32}$ I. Hristova, ${ }^{17}$ J. Hrivnac, ${ }^{119}$ T. Hryn'ova, ${ }^{5}$ A. Hrynevich, ${ }^{96}$ P. J. Hsu, ${ }^{63}$ S.-C. Hsu, ${ }^{140}$ Q. Hu, ${ }^{36 a}$ S. Hu,${ }^{36 c}$ Y. Huang, ${ }^{35 a}$ Z. Hubacek, ${ }^{130}$ F. Hubaut, ${ }^{88}$ F. Huegging, ${ }^{23}$ T. B. Huffman, ${ }^{122}$ E. W. Hughes, ${ }^{38}$ G. Hughes,${ }^{75}$ M. Huhtinen, ${ }^{32}$ P. Huo, ${ }^{150}$ N. Huseynov, ${ }^{68, c}$ J. Huston, ${ }^{93}$ J. Huth,${ }^{59}$ G. Iacobucci, ${ }^{52}$ G. Iakovidis, ${ }^{27}$ I. Ibragimov, ${ }^{143}$ L. Iconomidou-Fayard, ${ }^{119}$ Z. Idrissi, ${ }^{137 e}$ P. Iengo, ${ }^{32}$ O. Igonkina, ${ }^{109, z}$

T. Iizawa, ${ }^{174}$ Y. Ikegami,${ }^{69} \mathrm{M}$. Ikeno, ${ }^{69}$ Y. Ilchenko, ${ }^{11, \text { aa }}$ D. Iliadis,${ }^{156} \mathrm{~N}$. Ilic, ${ }^{145} \mathrm{G}$. Introzzi, ${ }^{123 a, 123 b}$ P. Ioannou, ${ }^{9, a}$ M. Iodice, ${ }^{136 a} \mathrm{~K}$. Iordanidou, ${ }^{38} \mathrm{~V}$. Ippolito, ${ }^{59} \mathrm{M}$. F. Isacson, ${ }^{168} \mathrm{~N}$. Ishijima, ${ }^{120} \mathrm{M}$. Ishino, ${ }^{157} \mathrm{M}$. Ishitsuka, ${ }^{159} \mathrm{C}$. Issever, ${ }^{122}$ S. Istin, ${ }^{20 a}$ F. Ito, ${ }^{164}$ J. M. Iturbe Ponce, ${ }^{62 a}$ R. Iuppa, ${ }^{162 a, 162 b}$ H. Iwasaki, ${ }^{69}$ J. M. Izen, ${ }^{44}$ V. Izzo, ${ }^{106 a}$ S. Jabbar, ${ }^{3}$ P. Jackson, ${ }^{1}$ R. M. Jacobs, ${ }^{23}$ V. Jain, ${ }^{2}$ K. B. Jakobi, ${ }^{86}$ K. Jakobs, ${ }^{51}$ S. Jakobsen, ${ }^{65}$ T. Jakoubek, ${ }^{129}$ D. O. Jamin, ${ }^{116}$ D. K. Jana, ${ }^{82}$ R. Jansky, ${ }^{52}$ J. Janssen, ${ }^{23}$ M. Janus ${ }^{57}$ P. A. Janus, ${ }^{41 a}$ G. Jarlskog, ${ }^{84}$ N. Javadov, ${ }^{68, c}$ T. Javůrek,${ }^{51}$ M. Javurkova, ${ }^{51}$ F. Jeanneau, ${ }^{138}$ L. Jeanty, ${ }^{16}$ J. Jejelava, ${ }^{54 a, b b}$ A. Jelinskas, ${ }^{173}$ P. Jenni,,${ }^{51, c c}$ C. Jeske, ${ }^{173}$ S. Jézéquel, ${ }^{5}$ H. Ji, ${ }^{176}$ J. Jia, ${ }^{150}$ H. Jiang, ${ }^{67}$ Y. Jiang, ${ }^{36 a}$ Z. Jiang, ${ }^{145}$ S. Jiggins, ${ }^{81}$ J. Jimenez Pena, ${ }^{170}$ S. Jin, ${ }^{35 a}$ A. Jinaru, ${ }^{28 b}$ O. Jinnouchi, ${ }^{159}$ H. Jivan, ${ }^{147 c}$ P. Johansson, ${ }^{141}$ K. A. Johns, ${ }^{7}$ C. A. Johnson, ${ }^{64}$ W. J. Johnson, ${ }^{140}$ K. Jon-And, ${ }^{148 a, 148 b}$ R. W. L. Jones, ${ }^{75}$ S. D. Jones, ${ }^{151}$ S. Jones, ${ }^{7}$ T. J. Jones, ${ }^{77}$ J. Jongmanns, ${ }^{60 a}$ P. M. Jorge, ${ }^{128 a, 128 b}$ J. Jovicevic, ${ }^{163 a}$ X. Ju, ${ }^{176}$ A. Juste Rozas, ${ }^{13, w}$ M. K. Köhler, ${ }^{175}$ A. Kaczmarska, ${ }^{42}$ M. Kado, ${ }^{119}$ H. Kagan, ${ }^{113}$ M. Kagan, ${ }^{145}$ S. J. Kahn ${ }^{88}$ T. Kaji ${ }^{174}$ E. Kajomovitz, ${ }^{48}$ C. W. Kalderon, ${ }^{84}$ A. Kaluza ${ }^{86}$ S. Kama, ${ }^{43}$ A. Kamenshchikov, ${ }^{132}$ N. Kanaya ${ }^{157}$ L. Kanjir ${ }^{78}$ V. A. Kantserov, ${ }^{100}$ J. Kanzaki, ${ }^{69}$ B. Kaplan, ${ }^{112}$ L. S. Kaplan, ${ }^{176}$ D. Kar, ${ }^{147 c}$ K. Karakostas, ${ }^{10}$ N. Karastathis, ${ }^{10}$ M. J. Kareem, ${ }^{57}$ E. Karentzos, ${ }^{10}$ S. N. Karpov, ${ }^{68}$ Z. M. Karpova ${ }^{68}$ K. Karthik, ${ }^{112}$ V. Kartvelishvili, ${ }^{75}$ A. N. Karyukhin, ${ }^{132}$ K. Kasahara, ${ }^{164}$ L. Kashif, ${ }^{176}$ R. D. Kass ${ }^{113}$ A. Kastanas, ${ }^{149}$ Y. Kataoka, ${ }^{157}$ C. Kato, ${ }^{157}$ A. Katre,${ }^{52}$ J. Katzy, ${ }^{45}$ K. Kawade,${ }^{70}$ K. Kawagoe, ${ }^{73}$ T. Kawamoto, ${ }^{157}$ G. Kawamura, ${ }^{57}$ E. F. Kay, ${ }^{77}$ V. F. Kazanin, ${ }^{111, d}$ R. Keeler, ${ }^{172}$ R. Kehoe, ${ }^{43}$ J. S. Keller, ${ }^{31}$ E. Kellermann, ${ }^{84}$ J. J. Kempster, ${ }^{80}$ J Kendrick, ${ }^{19}$ H. Keoshkerian, ${ }^{161}$ O. Kepka, ${ }^{129}$ B. P. Kerševan, ${ }^{78}$ S. Kersten, ${ }^{178}$ R. A. Keyes,${ }^{90}$ M. Khader, ${ }^{169}$ F. Khalil-zada, ${ }^{12}$ A. Khanov, ${ }^{116}$ A. G. Kharlamov, ${ }^{111, d}$ T. Kharlamova, ${ }^{11, d}$ A. Khodinov, ${ }^{160}$ T. J. Khoo, ${ }^{52}$ V. Khovanskiy, ${ }^{99, a}$ E. Khramov ${ }^{68}$ J. Khubua, ${ }^{54 b \text {,dd }}$ S. Kido, ${ }^{70}$ C. R. Kilby, ${ }^{80}$ H. Y. Kim, ${ }^{8}$ S. H. Kim, ${ }^{164}$ Y. K. Kim, ${ }^{33}$ N. Kimura, ${ }^{156}$ O. M. Kind, ${ }^{17}$ B. T. King, ${ }^{77}$ D. Kirchmeier, ${ }^{47}$ J. Kirk, ${ }^{133}$ A. E. Kiryunin, ${ }^{103}$ T. Kishimoto, ${ }^{157}$ D. Kisielewska, ${ }^{41 a}$ V. Kitali, ${ }^{45}$ O. Kivernyk, ${ }^{5}$ E. Kladiva, ${ }^{146 \mathrm{~b}}$ T. Klapdor-Kleingrothaus, ${ }^{51}$ M. H. Klein, ${ }^{38}$ M. Klein,${ }^{77}$ U. Klein, ${ }^{77}$ K. Kleinknecht, ${ }^{86}$ P. Klimek,${ }^{10}$ A. Klimentov, ${ }^{27}$ R. Klingenberg, ${ }^{46}$ T. Klingl, ${ }^{23}$ T. Klioutchnikova, ${ }^{32}$ E.-E. Kluge, ${ }^{60 a}$ P. Kluit, ${ }^{109}$ S. Kluth,${ }^{103}$ E. Kneringer, ${ }^{65}$

E. B. F. G. Knoops ${ }^{88}$ A. Knue, ${ }^{103}$ A. Kobayashi, ${ }^{157}$ D. Kobayashi, ${ }^{159}$ T. Kobayashi, ${ }^{157}$ M. Kobel, ${ }^{47}$ M. Kocian, ${ }^{145}$ P. Kodys, ${ }^{131}$ T. Koffas, ${ }^{31}$ E. Koffeman, ${ }^{109}$ N. M. Köhler, ${ }^{103}$ T. Koi, ${ }^{145}$ M. Kolb, ${ }^{60 b}$ I. Koletsou, ${ }^{5}$ A. A. Komar, ${ }^{98, a}$ Y. Komori, ${ }^{157}$ T. Kondo, ${ }^{69}$ N. Kondrashova, ${ }^{36 \mathrm{c}}$ K. Köneke, ${ }^{51}$ A. C. König, ${ }^{108}$ T. Kono, ${ }^{69, e e}$ R. Konoplich, ${ }^{112, f f}$ N. Konstantinidis, ${ }^{81}$ R. Kopeliansky, ${ }^{64}$ S. Koperny, ${ }^{41 a}$ A. K. Kopp,${ }^{51}$ K. Korcyl, ${ }^{42}$ K. Kordas, ${ }^{156}$ A. Korn, ${ }^{81}$ A. A. Korol, ${ }^{11, d}$ I. Korolkov, ${ }^{13}$ E. V. Korolkova, ${ }^{141}$ O. Kortner, ${ }^{103}$ S. Kortner, ${ }^{103}$ T. Kosek, ${ }^{131}$ V. V. Kostyukhin, ${ }^{23}$ A. Kotwal, ${ }^{48}$

A. Koulouris, ${ }^{10}$ A. Kourkoumeli-Charalampidi, ${ }^{123 a, 123 b}$ C. Kourkoumelis ${ }^{9}$ E. Kourlitis, ${ }^{141}$ V. Kouskoura, ${ }^{27}$ A. B. Kowalewska, ${ }^{42}$ R. Kowalewski ${ }^{172}$ T.Z. Kowalski, ${ }^{41 a}$ C. Kozakai ${ }^{157}$ W. Kozanecki, ${ }^{138}$ A. S. Kozhin, ${ }^{132}$ V. A. Kramarenko, ${ }^{101}$ G. Kramberger, ${ }^{78}$ D. Krasnopevtsev, ${ }^{100}$ M. W. Krasny, ${ }^{83}$ A. Krasznahorkay, ${ }^{32}$ D. Krauss, ${ }^{103}$ J. A. Kremer, ${ }^{41 a}$ J. Kretzschmar, ${ }^{77}$ K. Kreutzfeldt, ${ }^{55}$ P. Krieger, ${ }^{161}$ K. Krizka, ${ }^{33}$ K. Kroeninger, ${ }^{46}$ H. Kroha, ${ }^{103}$ J. Kroll, ${ }^{129}$ J. Kroll, ${ }^{124}$ J. Kroseberg, ${ }^{23}$ J. Krstic, ${ }^{14}$ U. Kruchonak, ${ }^{68}$ H. Krüger,${ }^{23}$ N. Krumnack, ${ }^{67}$ M. C. Kruse, ${ }^{48}$ T. Kubota, ${ }^{91}$ H. Kucuk, ${ }^{81}$ S. Kuday, ${ }^{4 b}$ J. T. Kuechler, ${ }^{178}$ S. Kuehn, ${ }^{32}$ A. Kugel,${ }^{60 a}$ F. Kuger, ${ }^{177}$ T. Kuhl, ${ }^{45}$ V. Kukhtin, ${ }^{68}$ R. Kukla, ${ }^{88}$ 
Y. Kulchitsky, ${ }^{95}$ S. Kuleshov, ${ }^{34 b}$ Y. P. Kulinich, ${ }^{169}$ M. Kuna, ${ }^{134 a, 134 b}$ T. Kunigo, ${ }^{71}$ A. Kupco, ${ }^{129}$ T. Kupfer, ${ }^{46}$ O. Kuprash, ${ }^{155}$ H. Kurashige,${ }^{70}$ L. L. Kurchaninov, ${ }^{163 a}$ Y. A. Kurochkin, ${ }^{95}$ M. G. Kurth, ${ }^{35 a}$ V. Kus, ${ }^{129}$ E. S. Kuwertz, ${ }^{172}$ M. Kuze, ${ }^{159}$ J. Kvita, ${ }^{117}$ T. Kwan, ${ }^{172}$ D. Kyriazopoulos, ${ }^{141}$ A. La Rosa, ${ }^{103}$ J. L. La Rosa Navarro, ${ }^{26 \mathrm{~d}}$ L. La Rotonda, ${ }^{40 a, 40 \mathrm{~b}}$ F. La Ruffa, ${ }^{40 a, 40 b}$ C. Lacasta, ${ }^{170}$ F. Lacava, ${ }^{134 a, 134 b}$ J. Lacey, ${ }^{45}$ H. Lacker,${ }^{17}$ D. Lacour, ${ }^{83}$ E. Ladygin, ${ }^{68}$ R. Lafaye, ${ }^{5}$ B. Laforge, ${ }^{83}$ T. Lagouri, ${ }^{179}$ S. Lai, ${ }^{57}$ S. Lammers, ${ }^{64}$ W. Lampl, ${ }^{7}$ E. Lançon, ${ }^{27}$ U. Landgraf, ${ }^{51}$ M. P. J. Landon, ${ }^{79}$ M. C. Lanfermann, ${ }^{52}$ V. S. Lang, ${ }^{60 a}$ J. C. Lange, ${ }^{13}$ R. J. Langenberg, ${ }^{32}$ A. J. Lankford, ${ }^{166}$ F. Lanni, ${ }^{27}$ K. Lantzsch, ${ }^{23}$ A. Lanza, ${ }^{123 a}$ A. Lapertosa, ${ }^{53 a, 53 b}$ S. Laplace, ${ }^{83}$ J. F. Laporte, ${ }^{138}$ T. Lari, ${ }^{94 a}$ F. Lasagni Manghi, ${ }^{22 a, 22 b}$ M. Lassnig, ${ }^{32}$ T. S. Lau, ${ }^{62 a}$ P. Laurelli, ${ }^{50}$ W. Lavrijsen, ${ }^{16}$ A. T. Law, ${ }^{139}$ P. Laycock, ${ }^{77}$ T. Lazovich, ${ }^{59}$ M. Lazzaroni, ${ }^{94 a, 94 b}$ B. Le, ${ }^{91}$ O. Le Dortz, ${ }^{83}$ E. Le Guirriec, ${ }^{88}$ E. P. Le Quilleuc, ${ }^{138}$ M. LeBlanc, ${ }^{172}$ T. LeCompte,${ }^{6}$ F. Ledroit-Guillon, ${ }^{58}$ C. A. Lee, ${ }^{27}$ G. R. Lee, ${ }^{133, g g}$ S. C. Lee, ${ }^{153}$ L. Lee, ${ }^{59}$ B. Lefebvre, ${ }^{90}$ G. Lefebvre,${ }^{83}$ M. Lefebvre, ${ }^{172}$ F. Legger, ${ }^{102}$ C. Leggett, ${ }^{16}$ G. Lehmann Miotto, ${ }^{32}$ X. Lei, ${ }^{7}$ W. A. Leight, ${ }^{45}$ M. A. L. Leite, ${ }^{26 \mathrm{~d}}$ R. Leitner, ${ }^{131}$ D. Lellouch, ${ }^{175}$ B. Lemmer, ${ }^{57}$ K. J. C. Leney, ${ }^{81}$ T. Lenz, ${ }^{23}$ B. Lenzi, ${ }^{32}$ R. Leone, ${ }^{7}$ S. Leone, ${ }^{126 a, 126 b}$ C. Leonidopoulos, ${ }^{49}$ G. Lerner, ${ }^{151}$ C. Leroy, ${ }^{97}$ A. A. J. Lesage, ${ }^{138}$ C. G. Lester, ${ }^{30}$ M. Levchenko, ${ }^{125}$ J. Levêque, ${ }^{5}$ D. Levin, ${ }^{92}$ L. J. Levinson, ${ }^{175}$ M. Levy, ${ }^{19}$ D. Lewis,${ }^{79}$

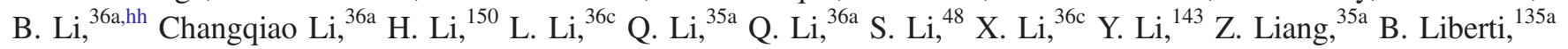
A. Liblong, ${ }^{161}$ K. Lie,${ }^{62 \mathrm{C}}$ J. Liebal, ${ }^{23}$ W. Liebig, ${ }^{15}$ A. Limosani, ${ }^{152}$ S. C. Lin, ${ }^{182}$ T. H. Lin,${ }^{86}$ B. E. Lindquist, ${ }^{150}$ A. E. Lionti,${ }^{52}$ E. Lipeles, ${ }^{124}$ A. Lipniacka, ${ }^{15}$ M. Lisovyi, ${ }^{60 b}$ T. M. Liss, ${ }^{169, \text { ii }}$ A. Lister,${ }^{171}$ A. M. Litke, ${ }^{139}$ B. Liu, ${ }^{67}$ H. Liu,${ }^{92}$ H. Liu, ${ }^{27}$ J. K. K. Liu, ${ }^{122}$ J. Liu, ${ }^{36 b}$ J. B. Liu, ${ }^{36 a}$ K. Liu, ${ }^{88}$ L. Liu, ${ }^{169}$ M. Liu, ${ }^{36 a}$ Y. L. Liu, ${ }^{36 a}$ Y. Liu, ${ }^{36 a}$ M. Livan, ${ }^{123 a, 123 b}$ A. Lleres,${ }^{58}$ J. Llorente Merino, ${ }^{35 \mathrm{a}}$ S. L. Lloyd, ${ }^{79}$ C. Y. Lo, ${ }^{62 b}$ F. Lo Sterzo, ${ }^{153}$ E. M. Lobodzinska, ${ }^{45}$ P. Loch, ${ }^{7}$ F. K. Loebinger, ${ }^{87}$ A. Loesle, ${ }^{51}$ K. M. Loew, ${ }^{25}$ A. Loginov, ${ }^{179, a}$ T. Lohse, ${ }^{17}$ K. Lohwasser, ${ }^{141}$ M. Lokajicek, ${ }^{129}$ B. A. Long, ${ }^{24}$ J. D. Long, ${ }^{169}$ R. E. Long, ${ }^{75}$ L. Longo, ${ }^{76 a, 76 b}$ K. A. Looper, ${ }^{113}$ J. A. Lopez, ${ }^{34 b}$ D. Lopez Mateos,${ }^{59}$ I. Lopez Paz, ${ }^{13}$ A. Lopez Solis, ${ }^{83}$ J. Lorenz, ${ }^{102}$ N. Lorenzo Martinez, ${ }^{5}$ M. Losada, ${ }^{21}$ P. J. Lösel, ${ }^{102}$ X. Lou, ${ }^{35 a}$ A. Lounis, ${ }^{119}$ J. Love, ${ }^{6}$ P. A. Love, ${ }^{75}$ H. Lu, ${ }^{62 a}$ N. Lu, ${ }^{92}$ Y. J. Lu ${ }^{63}$ H. J. Lubatti, ${ }^{140}$ C. Luci,${ }^{134 a, 134 b}$ A. Lucotte, ${ }^{58}$ C. Luedtke,${ }^{51}$ F. Luehring, ${ }^{64}$ W. Lukas, ${ }^{65}$ L. Luminari, ${ }^{134 a}$ O. Lundberg, ${ }^{148 a, 148 b}$ B. Lund-Jensen, ${ }^{149}$ M. S. Lutz, ${ }^{89}$ P. M. Luzi,${ }^{83}$ D. Lynn, ${ }^{27}$ R. Lysak, ${ }^{129}$ E. Lytken,${ }^{84}$ F. Lyu, ${ }^{35 a}$ V. Lyubushkin, ${ }^{68}$ H. Ma ${ }^{27}$ L. L. Ma, ${ }^{36 \mathrm{~b}}$ Y. Ma ${ }^{36 \mathrm{~b}}$ G. Maccarrone, ${ }^{50}$ A. Macchiolo, ${ }^{103}$ C. M. Macdonald, ${ }^{141}$ B. Maček, ${ }^{78}$ J. Machado Miguens, ${ }^{124,128 b}$ D. Madaffari, ${ }^{170}$ R. Madar, ${ }^{37}$ W. F. Mader, ${ }^{47}$ A. Madsen, ${ }^{45}$ J. Maeda, ${ }^{70}$ S. Maeland, ${ }^{15}$

T. Maeno, ${ }^{27}$ A. S. Maevskiy, ${ }^{101}$ V. Magerl, ${ }^{51}$ J. Mahlstedt, ${ }^{109}$ C. Maiani, ${ }^{119}$ C. Maidantchik, ${ }^{26 a}$ A. A. Maier, ${ }^{103}$ T. Maier, ${ }^{102}$ A. Maio, ${ }^{128 a, 128 b, 128 \mathrm{~d}}$ O. Majersky, ${ }^{146 \mathrm{a}}$ S. Majewski, ${ }^{118}$ Y. Makida, ${ }^{69}$ N. Makovec, ${ }^{119}$ B. Malaescu, ${ }^{83}$ Pa. Malecki, ${ }^{42}$ V. P. Maleev, ${ }^{125}$ F. Malek,${ }^{58}$ U. Mallik, ${ }^{66}$ D. Malon,${ }^{6}$ C. Malone,${ }^{30}$ S. Maltezos, ${ }^{10}$ S. Malyukov,${ }^{32}$ J. Mamuzic, ${ }^{170}$ G. Mancini, ${ }^{50}$ I. Mandić, ${ }^{78}$ J. Maneira, ${ }^{128 a, 128 b}$ L. Manhaes de Andrade Filho, ${ }^{26 \mathrm{~b}}$ J. Manjarres Ramos, ${ }^{47}$ K. H. Mankinen, ${ }^{84}$ A. Mann ${ }^{102}$ A. Manousos, ${ }^{32}$ B. Mansoulie, ${ }^{138}$ J. D. Mansour, ${ }^{35 a}$ R. Mantifel,${ }^{90}$ M. Mantoani,${ }^{57}$ S. Manzoni, ${ }^{94 a, 94 b}$ L. Mapelli, ${ }^{32}$ G. Marceca, ${ }^{29}$ L. March,${ }^{52}$ L. Marchese, ${ }^{122}$ G. Marchiori, ${ }^{83}$ M. Marcisovsky, ${ }^{129}$ M. Marjanovic, ${ }^{37}$ D. E. Marley, ${ }^{92}$ F. Marroquim, ${ }^{26 a}$ S. P. Marsden, ${ }^{87}$ Z. Marshall, ${ }^{16}$ M. U. F Martensson, ${ }^{168}$ S. Marti-Garcia, ${ }^{170}$ C. B. Martin, ${ }^{113}$ T. A. Martin, ${ }^{173}$ V. J. Martin, ${ }^{49}$ B. Martin dit Latour, ${ }^{15}$ M. Martinez, ${ }^{13, w}$ V. I. Martinez Outschoorn, ${ }^{169}$ S. Martin-Haugh, ${ }^{133}$ V. S. Martoiu, ${ }^{28 b}$ A. C. Martyniuk, ${ }^{81}$ A. Marzin, ${ }^{32}$ L. Masetti ${ }^{86}$ T. Mashimo, ${ }^{157}$ R. Mashinistov, ${ }^{98}$ J. Masik, ${ }^{87}$ A. L. Maslennikov, ${ }^{111, \mathrm{~d}}$ L. Massa, ${ }^{135 a, 135 \mathrm{~b}}$ P. Mastrandrea, ${ }^{5}$ A. Mastroberardino, ${ }^{40 a, 40 \mathrm{~b}}$ T. Masubuchi, ${ }^{157}$ P. Mättig, ${ }^{178}$ J. Maurer, ${ }^{28 b}$ S. J. Maxfield, ${ }^{77}$ D. A. Maximov, ${ }^{11, d}$ R. Mazini, ${ }^{153}$ I. Maznas, ${ }^{156}$ S. M. Mazza, ${ }^{94 a, 94 b}$ N. C. Mc Fadden, ${ }^{107}$ G. Mc Goldrick, ${ }^{161}$ S. P. Mc Kee, ${ }^{92}$ A. McCarn, ${ }^{92}$ R. L. McCarthy, ${ }^{150}$ T. G. McCarthy, ${ }^{103}$ L. I. McClymont, ${ }^{81}$ E. F. McDonald, ${ }^{91}$ J. A. Mcfayden,${ }^{32}$ G. Mchedlidze, ${ }^{57}$ S. J. McMahon, ${ }^{133}$ P. C. McNamara, ${ }^{91}$ R. A. McPherson, ${ }^{172, p}$ S. Meehan, ${ }^{140}$ T. J. Megy, ${ }^{51}$ S. Mehlhase, ${ }^{102}$ A. Mehta, ${ }^{77}$ T. Meideck ${ }^{58}$ K. Meier, ${ }^{60 a}$ B. Meirose, ${ }^{44}$ D. Melini, ${ }^{170, j j}$ B. R. Mellado Garcia, ${ }^{147 \mathrm{c}}$ J. D. Mellenthin,${ }^{57}$ M. Melo, ${ }^{146 \mathrm{a}}$ F. Meloni, ${ }^{18}$ A. Melzer, ${ }^{23}$ S. B. Menary, ${ }^{87}$ L. Meng, ${ }^{77}$ X. T. Meng, ${ }^{92}$ A. Mengarelli, ${ }^{2 a, 22 b}$ S. Menke, ${ }^{103}$ E. Meoni, ${ }^{40 a, 40 b}$ S. Mergelmeyer, ${ }^{17}$ C. Merlassino, ${ }^{18}$ P. Mermod, ${ }^{52}$ L. Merola, ${ }^{106 a, 106 b}$ C. Meroni, ${ }^{94 a}$ F. S. Merritt, ${ }^{33}$ A. Messina, ${ }^{134 a, 134 b}$ J. Metcalfe, ${ }^{6}$ A. S. Mete, ${ }^{166}$ C. Meyer, ${ }^{124}$ J-P. Meyer, ${ }^{138}$

J. Meyer, ${ }^{109}$ H. Meyer Zu Theenhausen, ${ }^{60 a}$ F. Miano, ${ }^{151}$ R. P. Middleton, ${ }^{133}$ S. Miglioranzi, ${ }^{53 a, 53 b}$ L. Mijović, ${ }^{49}$ G. Mikenberg, ${ }^{175}$ M. Mikestikova, ${ }^{129}$ M. Mikuž, ${ }^{78}$ M. Milesi, ${ }^{91}$ A. Milic, ${ }^{161}$ D. A. Millar, ${ }^{79}$ D. W. Miller, ${ }^{33}$ C. Mills, ${ }^{49}$ A. Milov, ${ }^{175}$ D. A. Milstead, ${ }^{148 a, 148 b}$ A. A. Minaenko, ${ }^{132}$ Y. Minami, ${ }^{157}$ I. A. Minashvili, ${ }^{68}$ A. I. Mincer, ${ }^{112}$ B. Mindur, ${ }^{41 a}$ M. Mineev, ${ }^{68}$ Y. Minegishi, ${ }^{157}$ Y. Ming, ${ }^{176}$ L. M. Mir, ${ }^{13}$ K. P. Mistry, ${ }^{124}$ T. Mitani, ${ }^{174}$ J. Mitrevski, ${ }^{102}$ V. A. Mitsou, ${ }^{170}$ A. Miucci, ${ }^{18}$ P. S. Miyagawa, ${ }^{141}$ A. Mizukami, ${ }^{69}$ J. U. Mjörnmark ${ }^{84}$ T. Mkrtchyan,${ }^{180}$ M. Mlynarikova, ${ }^{131}$ T. Moa, ${ }^{148 a, 148 b}$ K. Mochizuki, ${ }^{97}$ P. Mogg, ${ }^{51}$ S. Mohapatra, ${ }^{38}$ S. Molander ${ }^{148 a, 148 b}$ R. Moles-Valls, ${ }^{23}$ M. C. Mondragon, ${ }^{93}$ K. Mönig, ${ }^{45}$ J. Monk, ${ }^{39}$ E. Monnier, ${ }^{88}$ A. Montalbano, ${ }^{150}$ J. Montejo Berlingen, ${ }^{32}$ F. Monticelli, ${ }^{74}$ S. Monzani, ${ }^{94 a, 94 b}$ R. W. Moore, ${ }^{3}$ 
N. Morange, ${ }^{119}$ D. Moreno, ${ }^{21}$ M. Moreno Llácer, ${ }^{32}$ P. Morettini, ${ }^{53 a}$ S. Morgenstern, ${ }^{32}$ D. Mori, ${ }^{144}$ T. Mori, ${ }^{157}$ M. Morii, ${ }^{59}$ M. Morinaga ${ }^{174}$ V. Morisbak, ${ }^{121}$ A. K. Morley, ${ }^{32}$ G. Mornacchi,${ }^{32}$ J. D. Morris ${ }^{79}$ L. Morvaj, ${ }^{150}$ P. Moschovakos,${ }^{10}$ M. Mosidze ${ }^{54 \mathrm{~b}}$ H. J. Moss, ${ }^{141}$ J. Moss,${ }^{145, k \mathrm{k}}$ K. Motohashi, ${ }^{159}$ R. Mount, ${ }^{145}$ E. Mountricha, ${ }^{27}$ E. J. W. Moyse, ${ }^{89}$ S. Muanza, ${ }^{88}$ F. Mueller, ${ }^{103}$ J. Mueller, ${ }^{127}$ R. S. P. Mueller, ${ }^{102}$ D. Muenstermann, ${ }^{75}$ P. Mullen ${ }^{56}$ G. A. Mullier ${ }^{18}$ F. J. Munoz Sanchez, ${ }^{87}$ W. J. Murray, ${ }^{173,133}$ H. Musheghyan, ${ }^{32}$ M. Muškinja, ${ }^{78}$ A. G. Myagkov, ${ }^{132,11}$ M. Myska ${ }^{130}$ B. P. Nachman, ${ }^{16}$ O. Nackenhorst, ${ }^{52}$ K. Nagai, ${ }^{122}$ R. Nagai, ${ }^{69, \text { ee }}$ K. Nagano, ${ }^{69}$ Y. Nagasaka,${ }^{61}$ K. Nagata, ${ }^{164}$ M. Nagel,${ }^{51}$ E. Nagy, ${ }^{88}$ A. M. Nairz, ${ }^{32}$ Y. Nakahama, ${ }^{105}$ K. Nakamura, ${ }^{69}$ T. Nakamura, ${ }^{157}$ I. Nakano, ${ }^{114}$ R. F. Naranjo Garcia ${ }^{45}$ R. Narayan, ${ }^{11}$ D. I. Narrias Villar, ${ }^{60 a}$ I. Naryshkin, ${ }^{125}$ T. Naumann, ${ }^{45}$ G. Navarro, ${ }^{21}$ R. Nayyar, ${ }^{7}$ H. A. Neal,${ }^{92}$ P. Yu. Nechaeva, ${ }^{98}$ T. J. Neep, ${ }^{138}$ A. Negri, ${ }^{123 a, 123 b}$ M. Negrini, ${ }^{22 a}$ S. Nektarijevic, ${ }^{108}$ C. Nellist, ${ }^{19}$ A. Nelson, ${ }^{166}$ M. E. Nelson, ${ }^{122}$ S. Nemecek, ${ }^{129}$ P. Nemethy, ${ }^{112}$ M. Nessi, ${ }^{32, \mathrm{~mm}}$ M. S. Neubauer, ${ }^{169}$ M. Neumann, ${ }^{178}$ P. R. Newman, ${ }^{19}$ T. Y. Ng, ${ }^{62 \mathrm{c}}$ T. Nguyen Manh, ${ }^{97}$ R. B. Nickerson, ${ }^{122}$ R. Nicolaidou, ${ }^{138}$ J. Nielsen, ${ }^{139}$ V. Nikolaenko, ${ }^{132,11}$ I. Nikolic-Audit, ${ }^{83}$ K. Nikolopoulos, ${ }^{19}$ J. K. Nilsen, ${ }^{121}$ P. Nilsson, ${ }^{27}$ Y. Ninomiya, ${ }^{157}$ A. Nisati, ${ }^{134 a}$ N. Nishu, ${ }^{35 c}$ R. Nisius, ${ }^{103}$ I. Nitsche, ${ }^{46}$ T. Nitta, ${ }^{174}$ T. Nobe, ${ }^{157}$ Y. Noguchi, ${ }^{71}$ M. Nomachi, ${ }^{120}$ I. Nomidis, ${ }^{31}$ M. A. Nomura, ${ }^{27}$ T. Nooney, ${ }^{79}$ M. Nordberg, ${ }^{32}$ N. Norjoharuddeen, ${ }^{122}$ O. Novgorodova, ${ }^{47}$ S. Nowak, ${ }^{103}$ M. Nozaki, ${ }^{69}$ L. Nozka, ${ }^{117}$ K. Ntekas, ${ }^{166}$ E. Nurse,${ }^{81}$ F. Nuti,${ }^{91}$ K. O'connor, ${ }^{25}$ D. C. O'Neil, ${ }^{144}$ A. A. O'Rourke, ${ }^{45}$ V. O'Shea, ${ }^{56}$ F. G. Oakham, ${ }^{31, \mathrm{e}}$ H. Oberlack, ${ }^{103}$ T. Obermann, ${ }^{23}$ J. Ocariz, ${ }^{83}$ A. Ochi, ${ }^{70}$ I. Ochoa, ${ }^{38}$ J. P. Ochoa-Ricoux,${ }^{34 a}$ S. Oda, ${ }^{73}$ S. Odaka, ${ }^{69}$ A. Oh, ${ }^{87}$ S. H. Oh, ${ }^{48}$ C. C. Ohm, ${ }^{16}$ H. Ohman, ${ }^{168}$ H. Oide, ${ }^{53 a, 53 b}$ H. Okawa, ${ }^{164}$ Y. Okumura, ${ }^{157}$ T. Okuyama, ${ }^{69}$ A. Olariu, ${ }^{28 b}$ L. F. Oleiro Seabra, ${ }^{128 a}$ S. A. Olivares Pino, ${ }^{34 a}$ D. Oliveira Damazio, ${ }^{27}$ A. Olszewski, ${ }^{42}$ J. Olszowska ${ }^{42}$ A. Onofre, ${ }^{128 a, 128 e}$ K. Onogi, ${ }^{105}$ P. U. E. Onyisi, ${ }^{11, a a}$ H. Oppen, ${ }^{121}$ M. J. Oreglia, ${ }^{33}$ Y. Oren, ${ }^{155}$ D. Orestano, ${ }^{136 a, 136 b}$ N. Orlando, ${ }^{62 b}$ R. S. Orr, ${ }^{161}$ B. Osculati, ${ }^{53 a, 53 b, a}$ R. Ospanov, ${ }^{36 a}$ G. Otero y Garzon, ${ }^{29}$ H. Otono, ${ }^{73}$ M. Ouchrif, ${ }^{137 d}$ F. Ould-Saada, ${ }^{121}$ A. Ouraou, ${ }^{138}$ K. P. Oussoren, ${ }^{109}$ Q. Ouyang, ${ }^{35 a}$ M. Owen, ${ }^{56}$ R. E. Owen, ${ }^{19}$ V. E. Ozcan, ${ }^{20 a}$ N. Ozturk, ${ }^{8}$ K. Pachal, ${ }^{144}$ A. Pacheco Pages, ${ }^{13}$ L. Pacheco Rodriguez, ${ }^{138}$ C. Padilla Aranda,${ }^{13}$ S. Pagan Griso,${ }^{16}$ M. Paganini,${ }^{179}$ F. Paige, ${ }^{27}$ G. Palacino, ${ }^{64}$ S. Palazzo, ${ }^{40 a}, 40 b$ S. Palestini, ${ }^{32}$ M. Palka, ${ }^{41 \mathrm{~b}}$ D. Pallin, ${ }^{37}$ E. St. Panagiotopoulou, ${ }^{10}$ I. Panagoulias, ${ }^{10}$ C. E. Pandini,${ }^{126 a, 126 b}$ J. G. Panduro Vazquez, ${ }^{80}$ P. Pani, ${ }^{32}$ S. Panitkin, ${ }^{27}$ D. Pantea, ${ }^{28 b}$ L. Paolozzi, ${ }^{52}$ Th. D. Papadopoulou, ${ }^{10}$ K. Papageorgiou, ${ }^{9, t}$ A. Paramonov, ${ }^{6}$ D. Paredes Hernandez, ${ }^{179}$ A. J. Parker, ${ }^{75}$ M. A. Parker, ${ }^{30}$ K. A. Parker, ${ }^{45}$ F. Parodi, ${ }^{53 a, 53 b}$ J. A. Parsons, ${ }^{38}$ U. Parzefall, ${ }^{51}$ V. R. Pascuzzi, ${ }^{161}$ J. M. Pasner, ${ }^{139}$ E. Pasqualucci, ${ }^{134 a}$ S. Passaggio,${ }^{53 a}$ Fr. Pastore, ${ }^{80}$ S. Pataraia,${ }^{86}$ J. R. Pater ${ }^{87}$ T. Pauly, ${ }^{32}$ B. Pearson, ${ }^{103}$ S. Pedraza Lopez,${ }^{170}$ R. Pedro, ${ }^{128 a, 128 b}$ S. V. Peleganchuk, ${ }^{111, d}$ O. Penc, ${ }^{129}$ C. Peng, ${ }^{35 a}$ H. Peng, ${ }^{36 a}$

J. Penwell, ${ }^{64}$ B. S. Peralva ${ }^{26 \mathrm{~b}}$ M. M. Perego, ${ }^{138}$ D. V. Perepelitsa, ${ }^{27}$ F. Peri, ${ }^{17}$ L. Perini, ${ }^{94 a, 94 b}$ H. Pernegger,${ }^{32}$ S. Perrella, ${ }^{106 a, 106 b}$ R. Peschke, ${ }^{45}$ V. D. Peshekhonov, ${ }^{68, a}$ K. Peters,${ }^{45}$ R. F. Y. Peters, ${ }^{87}$ B. A. Petersen, ${ }^{32}$ T. C. Petersen, ${ }^{39}$ E. Petit, ${ }^{58}$ A. Petridis, ${ }^{1}$ C. Petridou, ${ }^{156}$ P. Petroff, ${ }^{119}$ E. Petrolo, ${ }^{134 a}$ M. Petrov, ${ }^{122}$ F. Petrucci, ${ }^{136 a, 136 b}$ N. E. Pettersson, ${ }^{89}$ A. Peyaud, ${ }^{138}$ R. Pezoa, ${ }^{34 \mathrm{~b}}$ F. H. Phillips, ${ }^{93}$ P. W. Phillips, ${ }^{133}$ G. Piacquadio, ${ }^{150}$ E. Pianori, ${ }^{173}$ A. Picazio ${ }^{89}$ E. Piccaro, ${ }^{79}$ M. A. Pickering, ${ }^{122}$ R. Piegaia, ${ }^{29}$ J. E. Pilcher, ${ }^{33}$ A. D. Pilkington, ${ }^{87}$ A. W. J. Pin,${ }^{87}$ M. Pinamonti, ${ }^{135 a, 135 b}$ J. L. Pinfold, ${ }^{3}$ H. Pirumov, ${ }^{45}$ M. Pitt ${ }^{175}$ L. Plazak, ${ }^{146 a}$ M.-A. Pleier ${ }^{27}$ V. Pleskot,${ }^{86}$ E. Plotnikova, ${ }^{68}$ D. Pluth, ${ }^{67}$ P. Podberezko, ${ }^{111}$ R. Poettgen,${ }^{84}$ R. Poggi, ${ }^{123 a, 123 b}$ L. Poggioli, ${ }^{119}$ I. Pogrebnyak, ${ }^{93}$ D. Pohl,${ }^{23}$ G. Polesello, ${ }^{123 a}$ A. Poley, ${ }^{45}$ A. Policicchio, ${ }^{40 a, 40 b}$ R. Polifka, ${ }^{32}$ A. Polini,${ }^{22 a}$ C. S. Pollard,${ }^{56}$ V. Polychronakos,${ }^{27}$ K. Pommès, ${ }^{32}$ D. Ponomarenko, ${ }^{100}$ L. Pontecorvo, ${ }^{134 a}$ G. A. Popeneciu, ${ }^{28 d}$ S. Pospisil, ${ }^{130}$ K. Potamianos, ${ }^{16}$ I. N. Potrap, ${ }^{68}$ C. J. Potter, ${ }^{30}$ T. Poulsen, ${ }^{84}$ J. Poveda, ${ }^{32}$ M. E. Pozo Astigarraga, ${ }^{32}$ P. Pralavorio, ${ }^{88}$ A. Pranko, ${ }^{16}$ S. Prell, ${ }^{67}$ D. Price, ${ }^{87}$ M. Primavera, ${ }^{76 a}$ S. Prince,${ }^{90}$ N. Proklova, ${ }^{100}$ K. Prokofiev, ${ }^{62 \mathrm{c}}$ F. Prokoshin, ${ }^{34 \mathrm{~b}}$ S. Protopopescu, ${ }^{27}$ J. Proudfoot, ${ }^{6}$ M. Przybycien, ${ }^{41 \mathrm{a}}$ A. Puri, ${ }^{169}$ P. Puzo, ${ }^{119}$ J. Qian, ${ }^{92}$ G. Qin, ${ }^{56}$ Y. Qin, ${ }^{87}$ A. Quadt, ${ }^{57}$ M. Queitsch-Maitland, ${ }^{45}$ D. Quilty, ${ }^{56}$ S. Raddum, ${ }^{121}$ V. Radeka, ${ }^{27}$ V. Radescu, ${ }^{122}$ S. K. Radhakrishnan ${ }^{150}$ P. Radloff ${ }^{118}$ P. Rados, ${ }^{91}$ F. Ragusa, ${ }^{94 a, 94 b}$ G. Rahal,${ }^{181}$ J. A. Raine, ${ }^{87}$ S. Rajagopalan, ${ }^{27}$

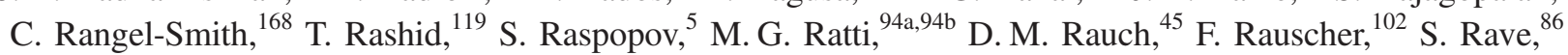
I. Ravinovich, ${ }^{175}$ J. H. Rawling, ${ }^{87}$ M. Raymond, ${ }^{32}$ A. L. Read, ${ }^{121}$ N. P. Readioff, ${ }^{58}$ M. Reale,${ }^{76 a, 76 b}$ D. M. Rebuzzi, ${ }^{123 a, 123 b}$ A. Redelbach, ${ }^{177}$ G. Redlinger, ${ }^{27}$ R. Reece, ${ }^{139}$ R. G. Reed, ${ }^{147 \mathrm{c}}$ K. Reeves, ${ }^{44}$ L. Rehnisch, ${ }^{17}$ J. Reichert, ${ }^{124}$ A. Reiss, ${ }^{86}$ C. Rembser ${ }^{32}$ H. Ren, ${ }^{35 a}$ M. Rescigno, ${ }^{134 a}$ S. Resconi, ${ }^{94 a}$ E. D. Resseguie, ${ }^{124}$ S. Rettie,${ }^{171}$ E. Reynolds,${ }^{19}$ O. L. Rezanova, ${ }^{111, \mathrm{~d}}$ P. Reznicek, ${ }^{131}$ R. Rezvani, ${ }^{97}$ R. Richter, ${ }^{103}$ S. Richter, ${ }^{81}$ E. Richter-Was, ${ }^{41 \mathrm{~b}}$ O. Ricken, ${ }^{23}$ M. Ridel, ${ }^{83}$ P. Rieck, ${ }^{103}$ C. J. Riegel, ${ }^{178}$ J. Rieger,${ }^{57}$ O. Rifki, ${ }^{115}$ M. Rijssenbeek, ${ }^{150}$ A. Rimoldi, ${ }^{123 a, 123 b}$ M. Rimoldi, ${ }^{18}$ L. Rinaldi, ${ }^{22 a}$ G. Ripellino, ${ }^{149}$ B. Ristić, ${ }^{32}$ E. Ritsch, ${ }^{32}$ I. Riu, ${ }^{13}$ F. Rizatdinova, ${ }^{116}$ E. Rizvi, ${ }^{79}$ C. Rizzi, ${ }^{13}$ R. T. Roberts,${ }^{87}$

S. H. Robertson, ${ }^{90, p}$ A. Robichaud-Veronneau, ${ }^{90}$ D. Robinson, ${ }^{30}$ J. E. M. Robinson, ${ }^{45}$ A. Robson, ${ }^{56}$ E. Rocco, ${ }^{86}$ C. Roda, ${ }^{126 a, 126 \mathrm{~b}}$ Y. Rodina, ${ }^{88, \mathrm{nn}}$ S. Rodriguez Bosca, ${ }^{170}$ A. Rodriguez Perez, ${ }^{13}$ D. Rodriguez Rodriguez, ${ }^{170}$ S. Roe, ${ }^{32}$ 
C. S. Rogan, ${ }^{59}$ O. Røhne, ${ }^{121}$ J. Roloff, ${ }^{59}$ A. Romaniouk,,${ }^{100}$ M. Romano, ${ }^{22 a, 22 b}$ S. M. Romano Saez,${ }^{37}$ E. Romero Adam, ${ }^{170}$ N. Rompotis, ${ }^{77}$ M. Ronzani,${ }^{51}$ L. Roos,${ }^{83}$ S. Rosati, ${ }^{134 a}$ K. Rosbach, ${ }^{51}$ P. Rose, ${ }^{139}$ N.-A. Rosien, ${ }^{57}$ E. Rossi, ${ }^{106 a, 106 b}$ L. P. Rossi, ${ }^{53 \mathrm{a}}$ J. H. N. Rosten, ${ }^{30}$ R. Rosten, ${ }^{140}$ M. Rotaru, ${ }^{28 \mathrm{~b}}$ J. Rothberg, ${ }^{140}$ D. Rousseau, ${ }^{119}$ A. Rozanov, ${ }^{88}$ Y. Rozen, ${ }^{154}$ X. Ruan, ${ }^{147 c}$ F. Rubbo, ${ }^{145}$ F. Rühr, ${ }^{51}$ A. Ruiz-Martinez,${ }^{31}$ Z. Rurikova, ${ }^{51}$ N. A. Rusakovich,${ }^{68}$ H. L. Russell, ${ }^{90}$ J. P. Rutherfoord, ${ }^{7}$ N. Ruthmann, ${ }^{32}$ Y. F. Ryabov, ${ }^{125}$ M. Rybar, ${ }^{169}$ G. Rybkin, ${ }^{119}$ S. Ryu, ${ }^{6}$ A. Ryzhov, ${ }^{132}$ G. F. Rzehorz,${ }^{57}$ A. F. Saavedra, ${ }^{152}$ G. Sabato, ${ }^{109}$ S. Sacerdoti, ${ }^{29}$ H. F-W. Sadrozinski, ${ }^{139}$ R. Sadykov, ${ }^{68}$ F. Safai Tehrani, ${ }^{134 a}$ P. Saha, ${ }^{110}$ M. Sahinsoy, ${ }^{60 \mathrm{a}}$ M. Saimpert ${ }^{45}$ M. Saito, ${ }^{157}$ T. Saito ${ }^{157}$ H. Sakamoto, ${ }^{157}$ Y. Sakurai, ${ }^{174}$ G. Salamanna, ${ }^{136 a, 136 b}$ J. E. Salazar Loyola, ${ }^{34 \mathrm{~b}}$ D. Salek, ${ }^{109}$ P. H. Sales De Bruin, ${ }^{168}$ D. Salihagic, ${ }^{103}$ A. Salnikov, ${ }^{145}$ J. Salt, ${ }^{170}$ D. Salvatore, ${ }^{40 a, 40 b}$ F. Salvatore, ${ }^{151}$ A. Salvucci, ${ }^{62 a, 62 b, 62 c}$ A. Salzburger, ${ }^{32}$ D. Sammel, ${ }^{51}$ D. Sampsonidis, ${ }^{156}$ D. Sampsonidou, ${ }^{156}$ J. Sánchez, ${ }^{170}$ V. Sanchez Martinez, ${ }^{170}$ A. Sanchez Pineda, ${ }^{167 a, 167 c}$ H. Sandaker, ${ }^{121}$ R. L. Sandbach, ${ }^{79}$ C. O. Sander, ${ }^{45}$ M. Sandhoff, ${ }^{178}$ C. Sandoval, ${ }^{21}$ D. P. C. Sankey, ${ }^{133}$ M. Sannino,${ }^{53 a, 53 b}$ Y. Sano, ${ }^{105}$ A. Sansoni,${ }^{50}$ C. Santoni, ${ }^{37}$ H. Santos, ${ }^{128 a}$ I. Santoyo Castillo, ${ }^{151}$ A. Sapronov, ${ }^{68}$ J. G. Saraiva, ${ }^{128 a, 128 d}$ B. Sarrazin, ${ }^{23}$ O. Sasaki,${ }^{69}$ K. Sato, ${ }^{164}$ E. Sauvan, ${ }^{5}$ G. Savage, ${ }^{80}$ P. Savard ${ }^{161, \mathrm{e}}$ N. Savic, ${ }^{103}$ C. Sawyer, ${ }^{133}$ L. Sawyer, ${ }^{82, v}$ J. Saxon, ${ }^{33}$ C. Sbarra, ${ }^{22 \mathrm{a}}$ A. Sbrizzi, ${ }^{22 a, 22 b}$ T. Scanlon, ${ }^{81}$ D. A. Scannicchio, ${ }^{166}$ J. Schaarschmidt, ${ }^{140}$ P. Schacht, ${ }^{103}$ B. M. Schachtner, ${ }^{102}$ D. Schaefer, ${ }^{32}$ L. Schaefer, ${ }^{124}$ R. Schaefer, ${ }^{45}$ J. Schaeffer, ${ }^{86}$ S. Schaepe, ${ }^{23}$ S. Schaetzel, ${ }^{60 b}$ U. Schäfer, ${ }^{86}$ A. C. Schaffer, ${ }^{119}$ D. Schaile, ${ }^{102}$ R. D. Schamberger ${ }^{150}$ V. A. Schegelsky, ${ }^{125}$ D. Scheirich, ${ }^{131}$ M. Schernau, ${ }^{166}$ C. Schiavi, ${ }^{53 a, 53 b}$ S. Schier, ${ }^{139}$ L. K. Schildgen, ${ }^{23}$ C. Schillo, ${ }^{51}$ M. Schioppa ${ }^{40 a, 40 b}$ S. Schlenker, ${ }^{32}$ K. R. Schmidt-Sommerfeld, ${ }^{103}$ K. Schmieden, ${ }^{32}$ C. Schmitt, ${ }^{86}$ S. Schmitt, ${ }^{45}$ S. Schmitz, ${ }^{86}$ U. Schnoor, ${ }^{51}$ L. Schoeffel, ${ }^{138}$ A. Schoening, ${ }^{60 b}$ B. D. Schoenrock, ${ }^{93}$ E. Schopf,${ }^{23}$ M. Schott, ${ }^{86}$ J. F. P. Schouwenberg, ${ }^{108}$ J. Schovancova, ${ }^{32}$ S. Schramm, ${ }^{52}$ N. Schuh, ${ }^{86}$ A. Schulte,${ }^{86}$ M. J. Schultens, ${ }^{23}$ H.-C. Schultz-Coulon, ${ }^{60 a}$ H. Schulz, ${ }^{17}$

M. Schumacher, ${ }^{51}$ B. A. Schumm, ${ }^{139}$ Ph. Schune, ${ }^{138}$ A. Schwartzman, ${ }^{145}$ T. A. Schwarz, ${ }^{92}$ H. Schweiger ${ }^{87}$

Ph. Schwemling, ${ }^{138}$ R. Schwienhorst, ${ }^{93}$ J. Schwindling, ${ }^{138}$ A. Sciandra, ${ }^{23}$ G. Sciolla, ${ }^{25}$ M. Scornajenghi, ${ }^{40 a, 40 b}$ F. Scuri, ${ }^{126 a, 126 b}$ F. Scutti, ${ }^{91}$ J. Searcy, ${ }^{92}$ P. Seema, ${ }^{23}$ S. C. Seidel, ${ }^{107}$ A. Seiden, ${ }^{139}$ J. M. Seixas, ${ }^{26 a}$ G. Sekhniaidze, ${ }^{106 a}$

K. Sekhon, ${ }^{92}$ S. J. Sekula, ${ }^{43}$ N. Semprini-Cesari, ${ }^{22 a, 22 b}$ S. Senkin, ${ }^{37}$ C. Serfon, ${ }^{121}$ L. Serin, ${ }^{119}$ L. Serkin,,${ }^{167 a, 167 b}$ M. Sessa, ${ }^{136 a, 136 b}$ R. Seuster, ${ }^{172}$ H. Severini, ${ }^{115}$ T. Sfiligoj, ${ }^{78}$ F. Sforza, ${ }^{165}$ A. Sfyrla, ${ }^{52}$ E. Shabalina, ${ }^{57}$ N. W. Shaikh, ${ }^{148 a, 148 b}$ L. Y. Shan, ${ }^{35 a}$ R. Shang, ${ }^{169}$ J. T. Shank,${ }^{24}$ M. Shapiro, ${ }^{16}$ P. B. Shatalov, ${ }^{99}$ K. Shaw, ${ }^{167 a, 167 b}$ S. M. Shaw,${ }^{87}$

A. Shcherbakova, ${ }^{148 a, 148 b}$ C. Y. Shehu, ${ }^{151}$ Y. Shen, ${ }^{115}$ N. Sherafati, ${ }^{31}$ P. Sherwood, ${ }^{81}$ L. Shi, ${ }^{153,00}$ S. Shimizu, ${ }^{70}$ C. O. Shimmin, ${ }^{179}$ M. Shimojima, ${ }^{104}$ I. P. J. Shipsey, ${ }^{122}$ S. Shirabe, ${ }^{73}$ M. Shiyakova, ${ }^{68, p p}$ J. Shlomi, ${ }^{175}$ A. Shmeleva, ${ }^{98}$ D. Shoaleh Saadi ${ }^{97}$ M. J. Shochet, ${ }^{33}$ S. Shojaii, ${ }^{94 a}$ D. R. Shope, ${ }^{115}$ S. Shrestha ${ }^{113}$ E. Shulga, ${ }^{100}$ M. A. Shupe,${ }^{7}$ P. Sicho, ${ }^{129}$ A. M. Sickles ${ }^{169}$ P. E. Sidebo, ${ }^{149}$ E. Sideras Haddad, ${ }^{147 \mathrm{c}}$ O. Sidiropoulou, ${ }^{177}$ A. Sidoti, ${ }^{22 a, 22 b}$ F. Siegert, ${ }^{47}$ Dj. Sijacki, ${ }^{14}$ J. Silva, ${ }^{128 a}, 128 d$ S. B. Silverstein, ${ }^{148 a}$ V. Simak, ${ }^{130}$ Lj. Simic, ${ }^{14}$ S. Simion, ${ }^{119}$ E. Simioni, ${ }^{86}$ B. Simmons, ${ }^{81}$ M. Simon, ${ }^{86}$ P. Sinervo, ${ }^{161}$ N. B. Sinev, ${ }^{118}$ M. Sioli, ${ }^{22 a, 22 b}$ G. Siragusa, ${ }^{177}$ I. Siral,${ }^{92}$ S. Yu. Sivoklokov, ${ }^{101}$ J. Sjölin, ${ }^{148 a, 148 b}$ M. B. Skinner,${ }^{75}$ P. Skubic, ${ }^{115}$ M. Slater, ${ }^{19}$ T. Slavicek, ${ }^{130}$ M. Slawinska, ${ }^{42}$ K. Sliwa, ${ }^{165}$ R. Slovak, ${ }^{131}$ V. Smakhtin, ${ }^{175}$ B. H. Smart, ${ }^{5}$ J. Smiesko, ${ }^{146 a}$ N. Smirnov, ${ }^{100}$ S. Yu. Smirnov, ${ }^{100}$ Y. Smirnov, ${ }^{100}$ L. N. Smirnova, ${ }^{101, q q}$ O. Smirnova, ${ }^{84}$ J. W. Smith, ${ }^{57}$ M. N. K. Smith, ${ }^{38}$ R. W. Smith, ${ }^{38}$ M. Smizanska, ${ }^{75}$ K. Smolek, ${ }^{130}$ A. A. Snesarev, ${ }^{98}$ I. M. Snyder, ${ }^{18}$ S. Snyder, ${ }^{27}$ R. Sobie, ${ }^{172, p}$ F. Socher, ${ }^{47}$ A. Soffer, ${ }^{155}$ A. Søgaard, ${ }^{49}$ D. A. Soh, ${ }^{153}$ G. Sokhrannyi, ${ }^{78}$ C. A. Solans Sanchez,${ }^{32}$ M. Solar, ${ }^{130}$ E. Yu. Soldatov, ${ }^{100}$ U. Soldevila, ${ }^{170}$ A. A. Solodkov, ${ }^{132}$ A. Soloshenko, ${ }^{68}$ O. V. Solovyanov, ${ }^{132}$ V. Solovyev, ${ }^{125}$ P. Sommer, ${ }^{51}$ H. Son, ${ }^{165}$ A. Sopczak, ${ }^{130}$ D. Sosa, ${ }^{60 b}$ C. L. Sotiropoulou, ${ }^{126 a, 126 b}$ R. Soualah, ${ }^{167 a, 167 \mathrm{c}}$ A. M. Soukharev, ${ }^{11, d}$ D. South, ${ }^{45}$

B. C. Sowden, ${ }^{80}$ S. Spagnolo, ${ }^{76 a, 76 b}$ M. Spalla, ${ }^{126 a, 126 b}$ M. Spangenberg, ${ }^{173}$ F. Spanò, ${ }^{80}$ D. Sperlich, ${ }^{17}$ F. Spettel,${ }^{103}$ T. M. Spieker, ${ }^{60 a}$ R. Spighi, ${ }^{22 a}$ G. Spigo, ${ }^{32}$ L. A. Spiller, ${ }^{91}$ M. Spousta, ${ }^{131}$ R. D. St. Denis, ${ }^{56, a}$ A. Stabile, ${ }^{94 a}$ R. Stamen, ${ }^{60 a}$ S. Stamm, ${ }^{17}$ E. Stanecka ${ }^{42}$ R. W. Stanek, ${ }^{6}$ C. Stanescu, ${ }^{136 a}$ M. M. Stanitzki, ${ }^{45}$ B. S. Stapf,${ }^{109}$ S. Stapnes, ${ }^{121}$ E. A. Starchenko, ${ }^{132}$ G. H. Stark, ${ }^{33}$ J. Stark, ${ }^{58}$ S. H Stark, ${ }^{39}$ P. Staroba, ${ }^{129}$ P. Starovoitov, ${ }^{60 a}$ S. Stärz, ${ }^{32}$ R. Staszewski, ${ }^{42}$ P. Steinberg, ${ }^{27}$ B. Stelzer, ${ }^{144}$ H. J. Stelzer, ${ }^{32}$ O. Stelzer-Chilton, ${ }^{163 a}$ H. Stenzel, ${ }^{55}$ G. A. Stewart, ${ }^{56}$ M. C. Stockton, ${ }^{118}$ M. Stoebe, ${ }^{90}$ G. Stoicea, ${ }^{28 b}$ P. Stolte,${ }^{57}$ S. Stonjek, ${ }^{103}$ A. R. Stradling, ${ }^{8}$ A. Straessner, ${ }^{47}$ M. E. Stramaglia, ${ }^{18}$ J. Strandberg, ${ }^{149}$ S. Strandberg, ${ }^{148 \mathrm{a}, 148 \mathrm{~b}}$ M. Strauss, ${ }^{115}$ P. Strizenec, ${ }^{146 \mathrm{~b}}$ R. Ströhmer, ${ }^{177}$ D. M. Strom, ${ }^{118}$ R. Stroynowski ${ }^{43}$ A. Strubig, ${ }^{49}$ S. A. Stucci, ${ }^{27}$ B. Stugu, ${ }^{15}$ N. A. Styles, ${ }^{45}$ D. Su, ${ }^{145}$ J. Su, ${ }^{127}$ S. Suchek, ${ }^{60 a}$ Y. Sugaya, ${ }^{120}$ M. Suk, ${ }^{130}$ V. V. Sulin, ${ }^{98}$ DMS Sultan, ${ }^{162 a, 162 b}$ S. Sultansoy, ${ }^{4 c}$ T. Sumida, ${ }^{71}$ S. Sun,${ }^{59}$ X. Sun, ${ }^{3}$ K. Suruliz,${ }^{151}$ C. J. E. Suster, ${ }^{152}$ M. R. Sutton, ${ }^{151}$ S. Suzuki, ${ }^{69}$ M. Svatos, ${ }^{129}$ M. Swiatlowski, ${ }^{33}$ S. P. Swift, ${ }^{2}$ I. Sykora, ${ }^{146 a}$ T. Sykora,,${ }^{131}$ D. Ta, ${ }^{51}$ K. Tackmann, ${ }^{45}$ J. Taenzer, ${ }^{155}$ A. Taffard, ${ }^{166}$ R. Tafirout, ${ }^{163 a}$ E. Tahirovic, ${ }^{79}$ N. Taiblum, ${ }^{155}$ H. Takai, ${ }^{27}$ R. Takashima,${ }^{72}$ E. H. Takasugi,${ }^{103}$ T. Takeshita, ${ }^{142}$ Y. Takubo, ${ }^{69}$ M. Talby, ${ }^{88}$ A. A. Talyshev, ${ }^{111, \mathrm{~d}}$ J. Tanaka, ${ }^{157}$ M. Tanaka, ${ }^{159}$ R. Tanaka, ${ }^{119}$ S. Tanaka, ${ }^{69}$ R. Tanioka, ${ }^{70}$ 
B. B. Tannenwald, ${ }^{113}$ S. Tapia Araya, ${ }^{34 b}$ S. Tapprogge, ${ }^{86}$ S. Tarem, ${ }^{154}$ G. F. Tartarelli, ${ }^{94 a}$ P. Tas, ${ }^{131}$ M. Tasevsky,${ }^{129}$ T. Tashiro, ${ }^{71}$ E. Tassi ${ }^{40 a, 40 b}$ A. Tavares Delgado, ${ }^{128 a, 128 b}$ Y. Tayalati, ${ }^{137}$ A. C. Taylor, ${ }^{107}$ A. J. Taylor ${ }^{49}$ G. N. Taylor, ${ }^{91}$ P. T. E. Taylor, ${ }^{91}$ W. Taylor, ${ }^{163 b}$ P. Teixeira-Dias, ${ }^{80}$ D. Temple, ${ }^{144}$ H. Ten Kate,${ }^{32}$ P. K. Teng, ${ }^{153}$ J. J. Teoh,${ }^{120}$ F. Tepel, ${ }^{178}$ S. Terada ${ }^{69}$ K. Terashi, ${ }^{157}$ J. Terron, ${ }^{85}$ S. Terzo, ${ }^{13}$ M. Testa, ${ }^{50}$ R. J. Teuscher, ${ }^{161, p}$ T. Theveneaux-Pelzer, ${ }^{88}$ F. Thiele, ${ }^{39}$ J. P. Thomas, ${ }^{19}$ J. Thomas-Wilsker, ${ }^{80}$ P. D. Thompson, ${ }^{19}$ A. S. Thompson, ${ }^{56}$ L. A. Thomsen,${ }^{179}$ E. Thomson, ${ }^{124}$ M. J. Tibbetts ${ }^{16}$ R. E. Ticse Torres, ${ }^{88}$ V. O. Tikhomirov, ${ }^{98, \text { rr }}$ Yu. A. Tikhonov, ${ }^{11, d}$ S. Timoshenko, ${ }^{100}$ P. Tipton, ${ }^{179}$ S. Tisserant,${ }^{88} \mathrm{~K}$. Todome, ${ }^{159} \mathrm{~S}$. Todorova-Nova, ${ }^{5} \mathrm{~S}$. Todt,${ }^{47} \mathrm{~J}$. Tojo, ${ }^{73} \mathrm{~S}$. Tokár, ${ }^{146 \mathrm{a}} \mathrm{K}$. Tokushuku, ${ }^{69} \mathrm{E}$. Tolley, ${ }^{59}$ L. Tomlinson, ${ }^{87}$ M. Tomoto, ${ }^{105}$ L. Tompkins, ${ }^{145, s 8}$ K. Toms,${ }^{107}$ B. Tong,${ }^{59}$ P. Tornambe,${ }^{51}$ E. Torrence, ${ }^{118}$ H. Torres,${ }^{47}$ E. Torró Pastor, ${ }^{140}$ J. Toth ${ }^{88, t t}$ F. Touchard ${ }^{88}$ D. R. Tovey, ${ }^{141}$ C. J. Treado, ${ }^{112}$ T. Trefzger, ${ }^{177}$ F. Tresoldi, ${ }^{151}$ A. Tricoli, ${ }^{27}$ I. M. Trigger, ${ }^{163 a}$ S. Trincaz-Duvoid, ${ }^{83}$ M. F. Tripiana, ${ }^{13}$ W. Trischuk, ${ }^{161}$ B. Trocmé, ${ }^{58}$ A. Trofymov,${ }^{45}$ C. Troncon, ${ }^{94 a}$ M. Trottier-McDonald, ${ }^{16}$ M. Trovatelli, ${ }^{172}$ L. Truong, ${ }^{147 b}$ M. Trzebinski, ${ }^{42}$ A. Trzupek, ${ }^{42}$ K. W. Tsang, ${ }^{62 a}$ J. C-L. Tseng, ${ }^{122}$ P. V. Tsiareshka,${ }^{95}$ G. Tsipolitis, ${ }^{10}$ N. Tsirintanis, ${ }^{9}$ S. Tsiskaridze, ${ }^{13}$ V. Tsiskaridze,${ }^{51}$ E. G. Tskhadadze,${ }^{54 a}$ K. M. Tsui, ${ }^{62 a}$ I. I. Tsukerman, ${ }^{99}$ V. Tsulaia, ${ }^{16}$ S. Tsuno, ${ }^{69}$ D. Tsybychev ${ }^{150} \mathrm{Y}$. Tu, ${ }^{62 \mathrm{~b}}$ A. Tudorache, ${ }^{28 \mathrm{~b}}$ V. Tudorache ${ }^{28 \mathrm{~b}}$ T. T. Tulbure, ${ }^{28 \mathrm{a}}$ A. N. Tuna ${ }^{59}$ S. A. Tupputi, ${ }^{22 a, 22 b}$ S. Turchikhin, ${ }^{68}$ D. Turgeman, ${ }^{175}$ I. Turk Cakir, ${ }^{4 b, u u}$ R. Turra, ${ }^{94 a}$ P. M. Tuts, ${ }^{38}$ G. Ucchielli, ${ }^{22 a, 22 b}$ I. Ueda, ${ }^{69}$ M. Ughetto, ${ }^{148 a, 148 b}$ F. Ukegawa, ${ }^{164}$ G. Unal, ${ }^{32}$ A. Undrus, ${ }^{27}$ G. Unel, ${ }^{166}$ F. C. Ungaro, ${ }^{91}$ Y. Unno, ${ }^{69}$ C. Unverdorben, ${ }^{102}$ J. Urban, ${ }^{146 \mathrm{~b}}$ P. Urquijo, ${ }^{91}$ P. Urrejola, ${ }^{86}$ G. Usai, ${ }^{8}$ J. Usui, ${ }^{69}$ L. Vacavant, ${ }^{88}$ V. Vacek, ${ }^{130}$ B. Vachon, ${ }^{90}$ K. O. H. Vadla, ${ }^{121}$ A. Vaidya,${ }^{81}$ C. Valderanis, ${ }^{102}$ E. Valdes Santurio, ${ }^{148 a, 148 b}$ M. Valente, ${ }^{52}$ S. Valentinetti, ${ }^{22 a, 22 b}$ A. Valero, ${ }^{170}$ L. Valéry, ${ }^{13}$ S. Valkar, ${ }^{131}$ A. Vallier, ${ }^{5}$ J. A. Valls Ferrer, ${ }^{170}$ W. Van Den Wollenberg, ${ }^{109}$ H. van der Graaf, ${ }^{109}$ P. van Gemmeren, ${ }^{6}$ J. Van Nieuwkoop, ${ }^{144}$ I. van Vulpen, ${ }^{109}$ M. C. van Woerden, ${ }^{109}$ M. Vanadia, ${ }^{135 a, 135 b}$ W. Vandelli, ${ }^{32}$ A. Vaniachine, ${ }^{160}$ P. Vankov, ${ }^{109}$ G. Vardanyan, ${ }^{180}$ R. Vari, ${ }^{134 \mathrm{a}}$ E. W. Varnes, ${ }^{7}$ C. Varni, ${ }^{53 a, 53 \mathrm{~b}}$ T. Varol, ${ }^{43}$ D. Varouchas, ${ }^{119}$ A. Vartapetian, ${ }^{8}$ K. E. Varvell, ${ }^{152}$ J. G. Vasquez, ${ }^{179}$ G. A. Vasquez, ${ }^{34 b}$ F. Vazeille, ${ }^{37}$ T. Vazquez Schroeder, ${ }^{90}$ J. Veatch,${ }^{57}$ V. Veeraraghavan, ${ }^{7}$ L. M. Velocee ${ }^{161}$ F. Veloso, ${ }^{128 a, 128 \mathrm{c}}$ S. Veneziano, ${ }^{134 \mathrm{a}}$ A. Ventura, ${ }^{76 a, 76 \mathrm{~b}}$ M. Venturi, ${ }^{172}$ N. Venturi, ${ }^{32}$ A. Venturini, ${ }^{25}$ V. Vercesi, ${ }^{123 a}$ M. Verducci, ${ }^{136 a, 136 b}$ W. Verkerke, ${ }^{109}$ A. T. Vermeulen, ${ }^{109}$ J. C. Vermeulen, ${ }^{109}$ M. C. Vetterli, ${ }^{14, e}$ N. Viaux Maira ${ }^{34 \mathrm{~b}}$ O. Viazlo,${ }^{84}$ I. Vichou, ${ }^{169, \mathrm{a}}$ T. Vickey, ${ }^{141}$ O. E. Vickey Boeriu, ${ }^{141}$ G. H. A. Viehhauser, ${ }^{122}$ S. Viel,${ }^{16}$ L. Vigani, ${ }^{122}$ M. Villa, ${ }^{22 a, 22 b}$ M. Villaplana Perez,${ }^{44 a, 94 b}$ E. Vilucchi, ${ }^{50}$ M. G. Vincter, ${ }^{31}$ V. B. Vinogradov, ${ }^{68}$ A. Vishwakarma, ${ }^{45}$ C. Vittori, ${ }^{22 a, 22 b}$ I. Vivarelli, ${ }^{151}$ S. Vlachos, ${ }^{10}$ M. Vogel, ${ }^{178}$ P. Vokac, ${ }^{130}$ G. Volpi, ${ }^{126 a, 126 b} \mathrm{H}$. von der Schmitt, ${ }^{103}$ E. von Toerne, ${ }^{23}$ V. Vorobel, ${ }^{131} \mathrm{~K}$. Vorobev, ${ }^{100} \mathrm{M}$. Vos, ${ }^{170} \mathrm{R}$. Voss, ${ }^{32}$ J. H. Vossebeld, ${ }^{77}$ N. Vranjes, ${ }^{14}$ M. Vranjes Milosavljevic, ${ }^{14}$ V. Vrba, ${ }^{130}$ M. Vreeswijk, ${ }^{109}$ R. Vuillermet, ${ }^{32}$ I. Vukotic, ${ }^{33}$ P. Wagner, ${ }^{23}$ W. Wagner, ${ }^{178}$ J. Wagner-Kuhr, ${ }^{102}$ H. Wahlberg, ${ }^{74}$ S. Wahrmund, ${ }^{47}$ J. Walder, ${ }^{75}$ R. Walker, ${ }^{102}$ W. Walkowiak, ${ }^{143}$ V. Wallangen, ${ }^{148 a, 148 b}$ C. Wang, ${ }^{35 b}$ C. Wang, ${ }^{36 b, v v}$ F. Wang, ${ }^{176}$ H. Wang, ${ }^{16}$ H. Wang, ${ }^{3}$ J. Wang, ${ }^{45}$ J. Wang, ${ }^{152}$ Q. Wang, ${ }^{115}$ R. Wang, ${ }^{6}$ S. M. Wang, ${ }^{153}$ T. Wang, ${ }^{38}$ W. Wang, ${ }^{153, w w}$ W. Wang, ${ }^{36 \mathrm{a}}$ Z. Wang, ${ }^{36 \mathrm{c}}$ C. Wanotayaroj, ${ }^{118}$ A. Warburton, ${ }^{90}$ C. P. Ward, ${ }^{30}$ D. R. Wardrope,${ }^{81}$ A. Washbrook, ${ }^{49}$ P. M. Watkins, ${ }^{19}$ A. T. Watson, ${ }^{19}$ M. F. Watson,,${ }^{19}$ G. Watts,${ }^{140}$ S. Watts ${ }^{87}$ B. M. Waugh ${ }^{81}$ A. F. Webb, ${ }^{11}$ S. Webb,${ }^{86}$ M. S. Weber, ${ }^{18}$ S. W. Weber, ${ }^{177}$ S. A. Weber, ${ }^{31}$ J. S. Webster, ${ }^{6}$ A. R. Weidberg, ${ }^{122}$ B. Weinert, ${ }^{64}$ J. Weingarten, ${ }^{57} \mathrm{M}$. Weirich, ${ }^{86} \mathrm{C}$. Weiser, ${ }^{51} \mathrm{H}$. Weits, ${ }^{109} \mathrm{P}$. S. Wells, ${ }^{32}$ T. Wenaus, ${ }^{27} \mathrm{~T}$. Wengler, ${ }^{32} \mathrm{~S}$. Wenig, ${ }^{32}$ N. Wermes, ${ }^{23}$ M. D. Werner, ${ }^{67}$ P. Werner, ${ }^{32}$ M. Wessels, ${ }^{60 a}$ T. D. Weston, ${ }^{18}$ K. Whalen, ${ }^{118}$ N. L. Whallon, ${ }^{140}$ A. M. Wharton, ${ }^{75}$ A. S. White, ${ }^{92}$ A. White, ${ }^{8}$ M. J. White, ${ }^{1}$ R. White, ${ }^{34 b}$ D. Whiteson, ${ }^{166}$ B. W. Whitmore, ${ }^{75}$ F. J. Wickens, ${ }^{133}$ W. Wiedenmann, ${ }^{176}$ M. Wielers, ${ }^{133}$ C. Wiglesworth ${ }^{39}$ L. A. M. Wiik-Fuchs, ${ }^{51}$ A. Wildauer, ${ }^{103}$ F. Wilk,${ }^{87}$ H. G. Wilkens, ${ }^{32}$ H. H. Williams, ${ }^{124}$ S. Williams, ${ }^{109}$ C. Willis, ${ }^{93}$ S. Willocq, ${ }^{89}$ J. A. Wilson, ${ }^{19}$ I. Wingerter-Seez,${ }^{5}$ E. Winkels, ${ }^{151}$ F. Winklmeier, ${ }^{118}$ O. J. Winston, ${ }^{151}$ B. T. Winter ${ }^{23}$ M. Wittgen, ${ }^{145}$ M. Wobisch,${ }^{82, v}$ T. M. H. Wolf, ${ }^{109}$ R. Wolff, ${ }^{88}$ M. W. Wolter, ${ }^{42}$ H. Wolters, ${ }^{128 a, 128 c}$ V. W. S. Wong, ${ }^{171}$ S. D. Worm, ${ }^{19}$ B. K. Wosiek, ${ }^{42}$ J. Wotschack,${ }^{32}$ K. W. Wozniak, ${ }^{42}$ M. Wu, ${ }^{33}$ S. L. Wu, ${ }^{176}$ X. Wu, ${ }^{52}$ Y. Wu, ${ }^{92}$ T. R. Wyatt, ${ }^{87}$ B. M. Wynne, ${ }^{49}$ S. Xella, ${ }^{39}$ Z. Xi, ${ }^{92}$ L. Xia, ${ }^{35 c}$ D. Xu, ${ }^{35 a}$ L. Xu, ${ }^{27}$ T. Xu, ${ }^{138}$ B. Yabsley, ${ }^{152}$ S. Yacoob,${ }^{147 a}$ D. Yamaguchi, ${ }^{159}$ Y. Yamaguchi, ${ }^{159}$ A. Yamamoto, ${ }^{69}$ S. Yamamoto, ${ }^{157}$ T. Yamanaka, ${ }^{157}$ F. Yamane, ${ }^{70}$ M. Yamatani, ${ }^{157}$ Y. Yamazaki, ${ }^{70}$ Z. Yan, ${ }^{24}$ H. Yang, ${ }^{36 c}$ H. Yang, ${ }^{16}$ Y. Yang, ${ }^{153}$ Z. Yang, ${ }^{15}$ W-M. Yao, ${ }^{16}$ Y. C. Yap, ${ }^{83}$ Y. Yasu, ${ }^{69}$ E. Yatsenko, ${ }^{5}$ K. H. Yau Wong, ${ }^{23}$ J. Ye ${ }^{43}$ S. Ye, ${ }^{27}$ I. Yeletskikh, ${ }^{68}$ E. Yigitbasi ${ }^{24}$ E. Yildirim,${ }^{86}$ K. Yorita, ${ }^{174}$ K. Yoshihara, ${ }^{124}$ C. Young, ${ }^{145}$ C. J. S. Young, ${ }^{32}$ J. Yu, ${ }^{8}$ J. Yu, ${ }^{67}$ S. P. Y. Yuen,${ }^{23}$ I. Yusuff, ${ }^{30, x x}$ B. Zabinski, ${ }^{42}$ G. Zacharis, ${ }^{10}$ R. Zaidan, ${ }^{13}$ A. M. Zaitsev, ${ }^{132,11}$ N. Zakharchuk, ${ }^{45}$ J. Zalieckas, ${ }^{15}$ A. Zaman, ${ }^{150}$ S. Zambito,${ }^{59}$ D. Zanzi, ${ }^{91}$ C. Zeitnitz, ${ }^{178}$ G. Zemaityte, ${ }^{122}$ A. Zemla, ${ }^{41 a}$ J. C. Zeng, ${ }^{169}$ Q. Zeng, ${ }^{145}$ O. Zenin, ${ }^{132}$ T. Ženiš, ${ }^{146 a}$ D. Zerwas, ${ }^{119}$ D. Zhang, ${ }^{92}$ F. Zhang, ${ }^{176}$ G. Zhang, ${ }^{36 a, y y}$ H. Zhang, ${ }^{35 b}$ J. Zhang, ${ }^{6}$ L. Zhang, ${ }^{51}$ L. Zhang, ${ }^{36 a}$ M. Zhang, ${ }^{169}$ P. Zhang, ${ }^{35 b}$ R. Zhang, ${ }^{23}$ R. Zhang, ${ }^{36 a, v v}$ X. Zhang, ${ }^{36 \mathrm{~b}}$ Y. Zhang, ${ }^{35 \mathrm{a}}$ Z. Zhang, ${ }^{119}$ X. Zhao, ${ }^{43}$ Y. Zhao, ${ }^{36 \mathrm{~b}, \mathrm{zz}}$ Z. Zhao, ${ }^{36 \mathrm{a}}$ A. Zhemchugov, ${ }^{68}$ 
B. Zhou, ${ }^{92}$ C. Zhou, ${ }^{176}$ L. Zhou, ${ }^{43}$ M. Zhou, ${ }^{35 a}$ M. Zhou, ${ }^{150}$ N. Zhou, ${ }^{35 c}$ C. G. Zhu, ${ }^{36 b}$ H. Zhu, ${ }^{35 a}$ J. Zhu, ${ }^{92}$ Y. Zhu, ${ }^{36 a}$ X. Zhuang, ${ }^{35 a}$ K. Zhukov, ${ }^{98}$ A. Zibell, ${ }^{177}$ D. Zieminska, ${ }^{64}$ N. I. Zimine, ${ }^{68}$ C. Zimmermann, ${ }^{86} \mathrm{~S} . Z^{2}$ immermann, ${ }^{51}$ Z. Zinonos, ${ }^{103}$ M. Zinser, ${ }^{86}$ M. Ziolkowski, ${ }^{143}$ L. Živković, ${ }^{14}$ G. Zobernig, ${ }^{176}$ A. Zoccoli, ${ }^{22 a, 22 b}$ R. Zou, ${ }^{33}$ M. zur Nedden, ${ }^{17}$ and L. Zwalinski ${ }^{32}$

(ATLAS Collaboration)

${ }^{1}$ Department of Physics, University of Adelaide, Adelaide, Australia

${ }^{2}$ Physics Department, SUNY Albany, Albany New York, USA

${ }^{3}$ Department of Physics, University of Alberta, Edmonton Alberta, Canada

${ }^{4 a}$ Department of Physics, Ankara University, Ankara, Turkey

${ }^{4 \mathrm{~b}}$ Istanbul Aydin University, Istanbul, Turkey

${ }^{4 \mathrm{c}}$ Division of Physics, TOBB University of Economics and Technology, Ankara, Turkey

${ }^{5}$ LAPP, CNRS/IN2P3 and Université Savoie Mont Blanc, Annecy-le-Vieux, France

${ }^{6}$ High Energy Physics Division, Argonne National Laboratory, Argonne Illinois, USA

${ }^{7}$ Department of Physics, University of Arizona, Tucson Arizona, USA

${ }^{8}$ Department of Physics, The University of Texas at Arlington, Arlington Texas, USA

${ }^{9}$ Physics Department, National and Kapodistrian University of Athens, Athens, Greece

${ }^{10}$ Physics Department, National Technical University of Athens, Zografou, Greece

${ }^{11}$ Department of Physics, The University of Texas at Austin, Austin Texas, USA

${ }^{12}$ Institute of Physics, Azerbaijan Academy of Sciences, Baku, Azerbaijan

${ }^{13}$ Institut de Física d'Altes Energies (IFAE), The Barcelona Institute of Science and Technology, Barcelona, Spain

${ }^{14}$ Institute of Physics, University of Belgrade, Belgrade, Serbia

${ }^{15}$ Department for Physics and Technology, University of Bergen, Bergen, Norway

${ }^{16}$ Physics Division, Lawrence Berkeley National Laboratory and University of California, Berkeley California, USA

${ }^{17}$ Department of Physics, Humboldt University, Berlin, Germany

${ }^{18}$ Albert Einstein Center for Fundamental Physics and Laboratory for High Energy Physics, University of Bern, Bern, Switzerland

${ }^{19}$ School of Physics and Astronomy, University of Birmingham, Birmingham, United Kingdom

${ }^{20 a}$ Department of Physics, Bogazici University, Istanbul, Turkey

${ }^{20 \mathrm{~b}}$ Department of Physics Engineering, Gaziantep University, Gaziantep, Turkey

${ }^{20 \mathrm{c}}$ Istanbul Bilgi University, Faculty of Engineering and Natural Sciences, Istanbul, Turkey

${ }^{20 \mathrm{~d}}$ Bahcesehir University, Faculty of Engineering and Natural Sciences, Istanbul, Turkey

${ }^{21}$ Centro de Investigaciones, Universidad Antonio Narino, Bogota, Colombia ${ }^{22 a}$ INFN Sezione di Bologna, Italy

${ }^{22 \mathrm{~b}}$ Dipartimento di Fisica e Astronomia, Università di Bologna, Bologna, Italy

${ }^{23}$ Physikalisches Institut, University of Bonn, Bonn, Germany

${ }^{24}$ Department of Physics, Boston University, Boston Massachusetts, USA

${ }^{25}$ Department of Physics, Brandeis University, Waltham Massachusetts, USA

${ }^{26 a}$ Universidade Federal do Rio De Janeiro COPPE/EE/IF, Rio de Janeiro, Brazil

${ }^{26 \mathrm{~b}}$ Electrical Circuits Department, Federal University of Juiz de Fora (UFJF), Juiz de Fora, Brazil

${ }^{26 c}$ Federal University of Sao Joao del Rei (UFSJ), Sao Joao del Rei, Brazil

${ }^{26 \mathrm{~d}}$ Instituto de Fisica, Universidade de Sao Paulo, Sao Paulo, Brazil

${ }^{27}$ Physics Department, Brookhaven National Laboratory, Upton New York, USA

${ }^{28 a}$ Transilvania University of Brasov, Brasov, Romania

${ }^{28 \mathrm{~b}}$ Horia Hulubei National Institute of Physics and Nuclear Engineering, Bucharest, Romania

${ }^{28 \mathrm{c}}$ Department of Physics, Alexandru Ioan Cuza University of Iasi, Iasi, Romania

${ }^{28 \mathrm{~d}}$ National Institute for Research and Development of Isotopic and Molecular Technologies, Physics Department, Cluj Napoca, Romania

${ }^{28 \mathrm{e}}$ University Politehnica Bucharest, Bucharest, Romania

${ }^{28 \mathrm{f}}$ West University in Timisoara, Timisoara, Romania

${ }^{29}$ Departamento de Física, Universidad de Buenos Aires, Buenos Aires, Argentina

${ }^{30}$ Cavendish Laboratory, University of Cambridge, Cambridge, United Kingdom

${ }^{31}$ Department of Physics, Carleton University, Ottawa Ontario, Canada

${ }^{32}$ CERN, Geneva, Switzerland

${ }^{33}$ Enrico Fermi Institute, University of Chicago, Chicago Illinois, USA

${ }^{34 a}$ Departamento de Física, Pontificia Universidad Católica de Chile, Santiago, Chile 
${ }^{34 \mathrm{~b}}$ Departamento de Física, Universidad Técnica Federico Santa María, Valparaíso, Chile

${ }^{35 a}$ Institute of High Energy Physics, Chinese Academy of Sciences, Beijing, China

${ }^{35 \mathrm{~b}}$ Department of Physics, Nanjing University, Jiangsu, China

${ }^{35 \mathrm{c}}$ Physics Department, Tsinghua University, Beijing 100084, China

${ }^{36 a}$ Department of Modern Physics and State Key Laboratory of Particle Detection and Electronics, University of Science and Technology of China, Anhui, China

${ }^{36 \mathrm{~b}}$ School of Physics, Shandong University, Shandong, China

${ }^{36 \mathrm{c}}$ Department of Physics and Astronomy, Key Laboratory for Particle Physics, Astrophysics and

Cosmology, Ministry of Education; Shanghai Key Laboratory for Particle Physics and Cosmology,

Shanghai Jiao Tong University, Shanghai(also at PKU-CHEP), China

${ }^{37}$ Université Clermont Auvergne, CNRS/IN2P3, LPC, Clermont-Ferrand, France

${ }^{38}$ Nevis Laboratory, Columbia University, Irvington New York, USA

${ }^{39}$ Niels Bohr Institute, University of Copenhagen, Kobenhavn, Denmark

${ }^{40 a}$ INFN Gruppo Collegato di Cosenza, Laboratori Nazionali di Frascati, Italy

${ }^{40 \mathrm{~b}}$ Dipartimento di Fisica, Università della Calabria, Rende, Italy

${ }^{41 \mathrm{a}}$ AGH University of Science and Technology,

Faculty of Physics and Applied Computer Science, Krakow, Poland

${ }^{41 \mathrm{~b}}$ Marian Smoluchowski Institute of Physics, Jagiellonian University, Krakow, Poland

${ }^{42}$ Institute of Nuclear Physics Polish Academy of Sciences, Krakow, Poland

${ }^{43}$ Physics Department, Southern Methodist University, Dallas Texas, USA

${ }^{44}$ Physics Department, University of Texas at Dallas, Richardson Texas, USA

${ }^{45}$ DESY, Hamburg and Zeuthen, Germany

${ }^{46}$ Lehrstuhl für Experimentelle Physik IV, Technische Universität Dortmund, Dortmund, Germany

${ }^{47}$ Institut für Kern- und Teilchenphysik, Technische Universität Dresden, Dresden, Germany

${ }^{48}$ Department of Physics, Duke University, Durham North Carolin, USA

${ }^{49}$ SUPA - School of Physics and Astronomy, University of Edinburgh, Edinburgh, United Kingdom

${ }^{50}$ INFN e Laboratori Nazionali di Frascati, Frascati, Italy

${ }^{51}$ Fakultät für Mathematik und Physik, Albert-Ludwigs-Universität, Freiburg, Germany

${ }^{52}$ Departement de Physique Nucleaire et Corpusculaire, Université de Genève, Geneva, Switzerland

${ }^{53 a}$ INFN Sezione di Genova, Italy

${ }^{53 \mathrm{~b}}$ Dipartimento di Fisica, Università di Genova, Genova, Italy

${ }^{54 \mathrm{a}}$ E. Andronikashvili Institute of Physics, Iv. Javakhishvili Tbilisi State University, Tbilisi, Georgia

${ }^{54 \mathrm{~b}}$ High Energy Physics Institute, Tbilisi State University, Tbilisi, Georgia

${ }^{55}$ II Physikalisches Institut, Justus-Liebig-Universität Giessen, Giessen, Germany

${ }^{56}$ SUPA - School of Physics and Astronomy, University of Glasgow, Glasgow, United Kingdom

${ }^{57}$ II Physikalisches Institut, Georg-August-Universität, Göttingen, Germany

${ }^{58}$ Laboratoire de Physique Subatomique et de Cosmologie, Université Grenoble-Alpes, CNRS/IN2P3, Grenoble, France

${ }^{59}$ Laboratory for Particle Physics and Cosmology, Harvard University, Cambridge Massachusetts, USA

${ }^{60 a}$ Kirchhoff-Institut für Physik, Ruprecht-Karls-Universität Heidelberg, Heidelberg, Germany

${ }^{60 \mathrm{~b}}$ Physikalisches Institut, Ruprecht-Karls-Universität Heidelberg, Heidelberg, Germany

${ }^{61}$ Faculty of Applied Information Science, Hiroshima Institute of Technology, Hiroshima, Japan

${ }^{62 \mathrm{a}}$ Department of Physics, The Chinese University of Hong Kong, Shatin, N.T., Hong Kong, China

${ }^{62 \mathrm{~b}}$ Department of Physics, The University of Hong Kong, Hong Kong, China

${ }^{62 \mathrm{c}}$ Department of Physics and Institute for Advanced Study, The Hong Kong University of Science and Technology, Clear Water Bay, Kowloon, Hong Kong, China

${ }^{63}$ Department of Physics, National Tsing Hua University, Taiwan, Taiwan

${ }^{64}$ Department of Physics, Indiana University, Bloomington Indiana, USA

${ }^{65}$ Institut für Astro- und Teilchenphysik, Leopold-Franzens-Universität, Innsbruck, Austria

${ }^{66}$ University of Iowa, Iowa City Iowa, USA

${ }^{67}$ Department of Physics and Astronomy, Iowa State University, Ames Iowa, USA

${ }^{68}$ Joint Institute for Nuclear Research, JINR Dubna, Dubna, Russia

${ }^{69}$ KEK, High Energy Accelerator Research Organization, Tsukuba, Japan

${ }^{70}$ Graduate School of Science, Kobe University, Kobe, Japan

${ }^{71}$ Faculty of Science, Kyoto University, Kyoto, Japan

${ }^{72}$ Kyoto University of Education, Kyoto, Japan

${ }^{73}$ Research Center for Advanced Particle Physics and Department of Physics, Kyushu University, Fukuoka, Japan

${ }^{74}$ Instituto de Física La Plata, Universidad Nacional de La Plata and CONICET, La Plata, Argentina

${ }^{75}$ Physics Department, Lancaster University, Lancaster, United Kingdom 


\author{
${ }^{76 \mathrm{a}}$ INFN Sezione di Lecce, Italy \\ ${ }^{76 \mathrm{~b}}$ Dipartimento di Matematica e Fisica, Università del Salento, Lecce, Italy \\ ${ }^{77}$ Oliver Lodge Laboratory, University of Liverpool, Liverpool, United Kingdom \\ ${ }^{78}$ Department of Experimental Particle Physics, Jožef Stefan Institute and Department of Physics, \\ University of Ljubljana, Ljubljana, Slovenia \\ ${ }^{79}$ School of Physics and Astronomy, Queen Mary University of London, London, United Kingdom \\ ${ }^{80}$ Department of Physics, Royal Holloway University of London, Surrey, United Kingdom \\ ${ }^{81}$ Department of Physics and Astronomy, University College London, London, United Kingdom \\ ${ }^{82}$ Louisiana Tech University, Ruston Louisiana, USA \\ ${ }^{83}$ Laboratoire de Physique Nucléaire et de Hautes Energies, UPMC and Université Paris-Diderot and \\ CNRS/IN2P3, Paris, France \\ ${ }^{84}$ Fysiska institutionen, Lunds universitet, Lund, Sweden \\ ${ }^{85}$ Departamento de Fisica Teorica C-15, Universidad Autonoma de Madrid, Madrid, Spain \\ ${ }^{86}$ Institut für Physik, Universität Mainz, Mainz, Germany \\ ${ }^{87}$ School of Physics and Astronomy, University of Manchester, Manchester, United Kingdom \\ ${ }^{88}$ CPPM, Aix-Marseille Université and CNRS/IN2P3, Marseille, France \\ ${ }^{89}$ Department of Physics, University of Massachusetts, Amherst Massachusetts, USA \\ ${ }^{90}$ Department of Physics, McGill University, Montreal Québec, Canada \\ ${ }^{91}$ School of Physics, University of Melbourne, Victoria, Australia \\ ${ }^{92}$ Department of Physics, The University of Michigan, Ann Arbor Michigan, USA \\ ${ }^{93}$ Department of Physics and Astronomy, Michigan State University, East Lansing Michigan, USA \\ ${ }^{94 a}$ INFN Sezione di Milano, Italy \\ ${ }^{94 \mathrm{~b}}$ Dipartimento di Fisica, Università di Milano, Milano, Italy \\ ${ }^{95}$ B.I. Stepanov Institute of Physics, National Academy of Sciences of Belarus, Minsk, Republic of Belarus \\ ${ }^{96}$ Research Institute for Nuclear Problems of Byelorussian State University, Minsk, Republic of Belarus \\ ${ }^{97}$ Group of Particle Physics, University of Montreal, Montreal Québec, Canada \\ ${ }^{98}$ P.N. Lebedev Physical Institute of the Russian Academy of Sciences, Moscow, Russia \\ ${ }^{99}$ Institute for Theoretical and Experimental Physics (ITEP), Moscow, Russia \\ ${ }^{100}$ National Research Nuclear University MEPhI, Moscow, Russia \\ ${ }^{101}$ D.V. Skobeltsyn Institute of Nuclear Physics, M.V. Lomonosov Moscow State University, \\ Moscow, Russia \\ ${ }^{102}$ Fakultät für Physik, Ludwig-Maximilians-Universität München, München, Germany \\ ${ }^{103}$ Max-Planck-Institut für Physik (Werner-Heisenberg-Institut), München, Germany \\ ${ }^{104}$ Nagasaki Institute of Applied Science, Nagasaki, Japan \\ ${ }^{105}$ Graduate School of Science and Kobayashi-Maskawa Institute, Nagoya University, Nagoya, Japan \\ ${ }^{106 a}$ INFN Sezione di Napoli, Italy \\ ${ }^{106 \mathrm{~b}}$ Dipartimento di Fisica, Università di Napoli, Napoli, Italy \\ ${ }^{107}$ Department of Physics and Astronomy, University of New Mexico, Albuquerque New Mexico, USA \\ ${ }^{108}$ Institute for Mathematics, Astrophysics and Particle Physics, Radboud University Nijmegen/Nikhef, \\ Nijmegen, Netherlands \\ ${ }^{109}$ Nikhef National Institute for Subatomic Physics and University of Amsterdam, Amsterdam, Netherlands \\ ${ }^{110}$ Department of Physics, Northern Illinois University, DeKalb Illinois, USA \\ ${ }^{111}$ Budker Institute of Nuclear Physics, SB RAS, Novosibirsk, Russia \\ ${ }^{112}$ Department of Physics, New York University, New York New York, USA \\ ${ }^{113}$ Ohio State University, Columbus Ohio, USA \\ ${ }^{114}$ Faculty of Science, Okayama University, Okayama, Japan \\ ${ }^{115}$ Homer L. Dodge Department of Physics and Astronomy, University of Oklahoma, \\ Norman Oklahoma, USA \\ ${ }^{116}$ Department of Physics, Oklahoma State University, Stillwater Oklahoma, USA \\ ${ }^{117}$ Palacký University, RCPTM, Olomouc, Czech Republic \\ ${ }^{118}$ Center for High Energy Physics, University of Oregon, Eugene Oregon, USA \\ ${ }^{119}$ LAL, Univ. Paris-Sud, CNRS/IN2P3, Université Paris-Saclay, Orsay, France \\ ${ }^{120}$ Graduate School of Science, Osaka University, Osaka, Japan \\ ${ }^{121}$ Department of Physics, University of Oslo, Oslo, Norway \\ ${ }^{122}$ Department of Physics, Oxford University, Oxford, United Kingdom \\ ${ }^{123 a}$ INFN Sezione di Pavia, Italy \\ ${ }^{123 \mathrm{~b}}$ Dipartimento di Fisica, Università di Pavia, Pavia, Italy \\ ${ }^{124}$ Department of Physics, University of Pennsylvania, Philadelphia Pennsylvania, USA \\ ${ }^{125}$ National Research Centre "Kurchatov Institute” B.P.Konstantinov Petersburg Nuclear Physics \\ Institute, St. Petersburg, Russia
}


${ }^{126 a}$ INFN Sezione di Pisa, Italy

${ }^{126 \mathrm{~b}}$ Dipartimento di Fisica E. Fermi, Università di Pisa, Pisa, Italy

${ }^{127}$ Department of Physics and Astronomy, University of Pittsburgh, Pittsburgh Pennsylvania, USA

${ }^{128 a}$ Laboratório de Instrumentação e Física Experimental de Partículas - LIP, Lisboa, Portugal

${ }^{128 \mathrm{~b}}$ Faculdade de Ciências, Universidade de Lisboa, Lisboa, Portugal

${ }^{128 c}$ Department of Physics, University of Coimbra, Coimbra, Portugal

${ }^{128 \mathrm{~d}}$ Centro de Física Nuclear da Universidade de Lisboa, Lisboa, Portugal

${ }^{128 \mathrm{e}}$ Departamento de Fisica, Universidade do Minho, Braga, Portugal

${ }^{128 \mathrm{f}}$ Departamento de Fisica Teorica y del Cosmos and CAFPE, Universidad de Granada, Granada, Spain

${ }^{128 \mathrm{~g}}$ Dep Fisica and CEFITEC of Faculdade de Ciencias e Tecnologia, Universidade Nova de Lisboa, Caparica, Portugal

${ }^{129}$ Institute of Physics, Academy of Sciences of the Czech Republic, Praha, Czech Republic

${ }^{130}$ Czech Technical University in Prague, Praha, Czech Republic

${ }^{131}$ Charles University, Faculty of Mathematics and Physics, Prague, Czech Republic

${ }^{132}$ State Research Center Institute for High Energy Physics (Protvino), NRC KI, Russia

${ }^{133}$ Particle Physics Department, Rutherford Appleton Laboratory, Didcot, United Kingdom

${ }^{134 a}$ INFN Sezione di Roma, Italy

${ }^{134 \mathrm{~b}}$ Dipartimento di Fisica, Sapienza Università di Roma, Roma, Italy

${ }^{135 a}$ INFN Sezione di Roma Tor Vergata, Italy

${ }^{135 b}$ Dipartimento di Fisica, Università di Roma Tor Vergata, Roma, Italy

${ }^{136 a}$ INFN Sezione di Roma Tre, Italy

${ }^{136 \mathrm{~b}}$ Dipartimento di Matematica e Fisica, Università Roma Tre, Roma, Italy

${ }^{137 a}$ Faculté des Sciences Ain Chock, Réseau Universitaire de Physique des Hautes Energies - Université Hassan II, Casablanca, Morocco

${ }^{137 \mathrm{~b}}$ Centre National de l'Energie des Sciences Techniques Nucleaires, Rabat, Morocco

${ }^{137 c}$ Faculté des Sciences Semlalia, Université Cadi Ayyad, LPHEA-Marrakech, Morocco

${ }^{137 \mathrm{~d}}$ Faculté des Sciences, Université Mohamed Premier and LPTPM, Oujda, Morocco

${ }^{137 \mathrm{e}}$ Faculté des sciences, Université Mohammed V, Rabat, Morocco

${ }^{138}$ DSM/IRFU (Institut de Recherches sur les Lois Fondamentales de l'Univers), CEA Saclay

(Commissariat à l'Energie Atomique et aux Energies Alternatives), Gif-sur-Yvette, France

${ }^{139}$ Santa Cruz, Institute for Particle Physics, University of California Santa Cruz, Santa Cruz California, USA

${ }^{140}$ Department of Physics, University of Washington, Seattle Washington, USA

${ }^{141}$ Department of Physics and Astronomy, University of Sheffield, Sheffield, United Kingdom

${ }^{142}$ Department of Physics, Shinshu University, Nagano, Japan

${ }^{143}$ Department Physik, Universität Siegen, Siegen, Germany

${ }^{144}$ Department of Physics, Simon Fraser University, Burnaby British Columbia, Canada

${ }^{145}$ SLAC National Accelerator Laboratory, Stanford California, USA

${ }^{146 a}$ Faculty of Mathematics, Physics \& Informatics, Comenius University, Bratislava, Slovak Republic

${ }^{146 \mathrm{~b}}$ Department of Subnuclear Physics, Institute of Experimental Physics of the Slovak Academy of Sciences, Kosice, Slovak Republic

${ }^{147 a}$ Department of Physics, University of Cape Town, Cape Town, South Africa

${ }^{147 \mathrm{~b}}$ Department of Physics, University of Johannesburg, Johannesburg, South Africa

${ }^{147 \mathrm{c}}$ School of Physics, University of the Witwatersrand, Johannesburg, South Africa

${ }^{148 a}$ Department of Physics, Stockholm University, Sweden

${ }^{14 \mathrm{~b}}$ The Oskar Klein Centre, Stockholm, Sweden

${ }^{149}$ Physics Department, Royal Institute of Technology, Stockholm, Sweden

${ }^{150}$ Departments of Physics \& Astronomy and Chemistry, Stony Brook University,

Stony Brook New York, USA

${ }^{151}$ Department of Physics and Astronomy, University of Sussex, Brighton, United Kingdom

${ }^{152}$ School of Physics, University of Sydney, Sydney, Australia

${ }^{153}$ Institute of Physics, Academia Sinica, Taipei, Taiwan

${ }^{154}$ Department of Physics, Technion: Israel Institute of Technology, Haifa, Israel

${ }^{155}$ Raymond and Beverly Sackler School of Physics and Astronomy, Tel Aviv University, Tel Aviv, Israel

${ }^{156}$ Department of Physics, Aristotle University of Thessaloniki, Thessaloniki, Greece

${ }^{157}$ International Center for Elementary Particle Physics and Department of Physics, The University of Tokyo, Tokyo, Japan

${ }^{158}$ Graduate School of Science and Technology, Tokyo Metropolitan University, Tokyo, Japan

${ }^{159}$ Department of Physics, Tokyo Institute of Technology, Tokyo, Japan 


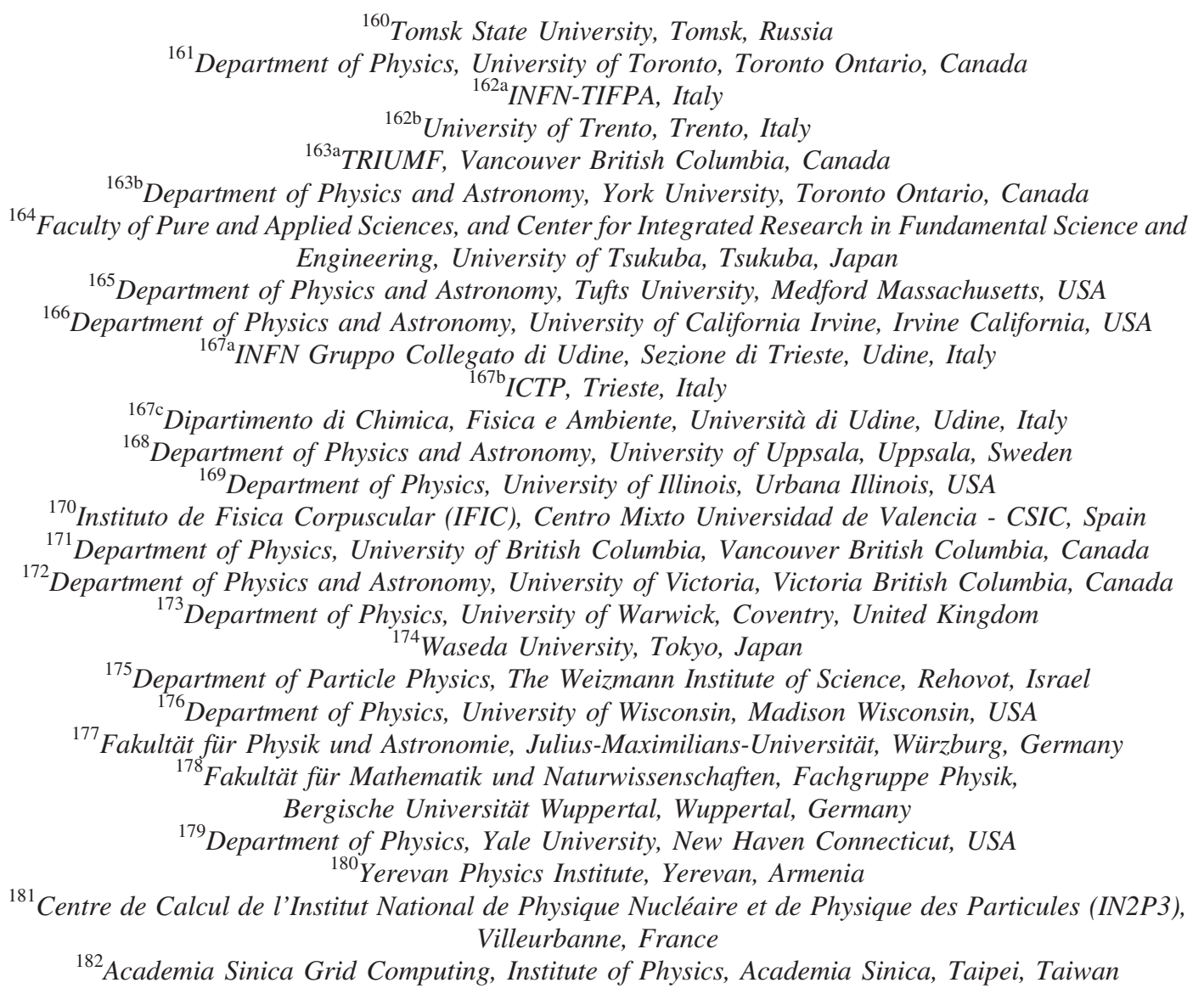

${ }^{\mathrm{a}}$ Deceased.

${ }^{\mathrm{b}}$ Also at Department of Physics, King's College London, London, United Kingdom.

${ }^{c}$ Also at Institute of Physics, Azerbaijan Academy of Sciences, Baku, Azerbaijan.

${ }^{\mathrm{d}}$ Also at Novosibirsk State University, Novosibirsk, Russia.

${ }^{\mathrm{e}}$ Also at TRIUMF, Vancouver BC, Canada.

${ }_{\mathrm{f}}^{\mathrm{f}}$ Also at Department of Physics \& Astronomy, University of Louisville, Louisville, KY, USA.

${ }^{g}$ Also at Physics Department, An-Najah National University, Nablus, Palestine.

${ }^{\mathrm{h}}$ Also at Department of Physics, California State University, Fresno CA, USA.

${ }^{1}$ Also at Department of Physics, University of Fribourg, Fribourg, Switzerland.

${ }^{\mathrm{j}}$ Also at II Physikalisches Institut, Georg-August-Universität, Göttingen, Germany.

${ }^{\mathrm{k}}$ Also at Departament de Fisica de la Universitat Autonoma de Barcelona, Barcelona, Spain.

${ }^{1}$ Also at Departamento de Fisica e Astronomia, Faculdade de Ciencias, Universidade do Porto, Portugal.

${ }^{\mathrm{m}}$ Also at Tomsk State University, Tomsk, Russia.

${ }^{\mathrm{n}}$ Also at The Collaborative Innovation Center of Quantum Matter (CICQM), Beijing, China.

${ }^{\circ}$ Also at Universita di Napoli Parthenope, Napoli, Italy.

${ }^{\mathrm{p}}$ Also at Institute of Particle Physics (IPP), Canada.

${ }^{\mathrm{q}}$ Also at Horia Hulubei National Institute of Physics and Nuclear Engineering, Bucharest, Romania.

${ }^{\mathrm{r}}$ Also at Department of Physics, St. Petersburg State Polytechnical University, St. Petersburg, Russia.

${ }^{\mathrm{s}}$ Also at Borough of Manhattan Community College, City University of New York, New York City, USA.

${ }^{t}$ Also at Department of Financial and Management Engineering, University of the Aegean, Chios, Greece.

${ }^{u}$ Also at Centre for High Performance Computing, CSIR Campus, Rosebank, Cape Town, South Africa.

${ }^{v}$ Also at Louisiana Tech University, Ruston LA, USA.

${ }^{\mathrm{w}}$ Also at Institucio Catalana de Recerca i Estudis Avancats, ICREA, Barcelona, Spain.

${ }^{\mathrm{x}}$ Also at Graduate School of Science, Osaka University, Osaka, Japan.

${ }^{y}$ Also at Fakultät für Mathematik und Physik, Albert-Ludwigs-Universität, Freiburg, Germany.

${ }^{\mathrm{z}}$ Also at Institute for Mathematics, Astrophysics and Particle Physics, Radboud University Nijmegen/Nikhef, Nijmegen, Netherlands.

${ }^{\text {aa } A l s o ~ a t ~ D e p a r t m e n t ~ o f ~ P h y s i c s, ~ T h e ~ U n i v e r s i t y ~ o f ~ T e x a s ~ a t ~ A u s t i n, ~ A u s t i n ~ T X, ~ U S A . ~}$

${ }^{\mathrm{bb}}$ Also at Institute of Theoretical Physics, Ilia State University, Tbilisi, Georgia. 
${ }^{c c}$ Also at CERN, Geneva, Switzerland.

${ }^{\mathrm{dd}}$ Also at Georgian Technical University (GTU),Tbilisi, Georgia.

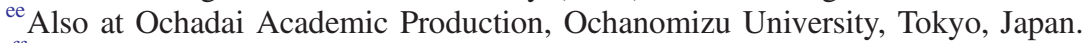

${ }^{\mathrm{ff}}$ Also at Manhattan College, New York NY, USA.

${ }^{g g}$ Also at Departamento de Física, Pontificia Universidad Católica de Chile, Santiago, Chile.

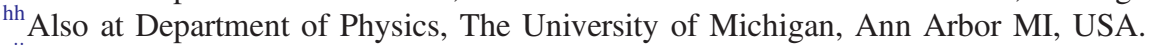

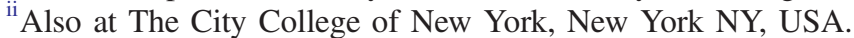

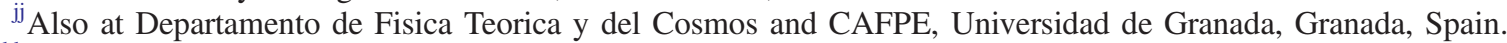

${ }^{\mathrm{kk}}$ Also at Department of Physics, California State University, Sacramento CA, USA.

${ }^{11}$ Also at Moscow Institute of Physics and Technology State University, Dolgoprudny, Russia.

${ }^{\mathrm{mm}}$ Also at Departement de Physique Nucleaire et Corpusculaire, Université de Genève, Geneva, Switzerland.

${ }^{n n}$ Also at Institut de Física d'Altes Energies (IFAE), The Barcelona Institute of Science and Technology, Barcelona, Spain.

${ }^{\circ o}$ Also at School of Physics, Sun Yat-sen University, Guangzhou, China.

${ }^{\mathrm{pp}}$ Also at Institute for Nuclear Research and Nuclear Energy (INRNE) of the Bulgarian Academy of Sciences, Sofia, Bulgaria.

${ }^{\mathrm{qq}}$ Also at Faculty of Physics, M.V.Lomonosov Moscow State University, Moscow, Russia.

${ }^{\text {rr }}$ Also at National Research Nuclear University MEPhI, Moscow, Russia.

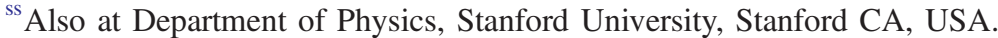

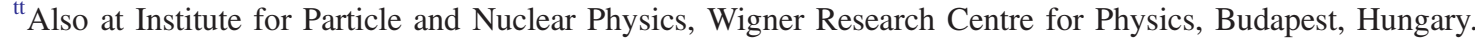

${ }^{\mathrm{uu}}$ Also at Giresun University, Faculty of Engineering, Turkey.

${ }^{\mathrm{vv}}$ Also at CPPM, Aix-Marseille Université and CNRS/IN2P3, Marseille, France.

${ }^{w w}$ Also at Department of Physics, Nanjing University, Jiangsu, China.

${ }^{\mathrm{xx}}$ Also at University of Malaya, Department of Physics, Kuala Lumpur, Malaysia.

${ }^{y y}$ Also at Institute of Physics, Academia Sinica, Taipei, Taiwan.

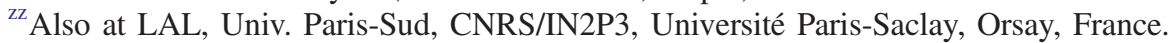

